Pacific

Journal of

Mathematics

\title{
RESURGENT DEFORMATIONS FOR AN ORDINARY DIFFERENTIAL EQUATION OF ORDER 2
}

ERIC Delabaere AND JEAN-MARC RASOAmANANA 


\title{
RESURGENT DEFORMATIONS FOR AN ORDINARY DIFFERENTIAL EQUATION OF ORDER 2
}

\author{
ERIC DELABAERE AND JEAN-MARC RASOAMANANA
}

\begin{abstract}
We consider the differential equation $\left(d^{2} / d x^{2}\right) \Phi(x)=\left(P_{m}(x) / x^{2}\right) \Phi(x)$ in the complex field, where $P_{m}$ is a monic polynomial function of order $\boldsymbol{m}$. We investigate the asymptotic and resurgent properties of the solutions at infinity, focusing in particular on the analytic dependence of the StokesSibuya multipliers on the coefficients of $\boldsymbol{P}_{\boldsymbol{m}}$. Taking into account the nontrivial monodromy at the origin, we derive a set of functional equations for the Stokes-Sibuya multipliers, and show how these relations can be used to compute the Stokes multipliers for a class of polynomials $\boldsymbol{P}_{\boldsymbol{m}}$. In particular, we obtain conditions for isomonodromic deformations when $m=3$.
\end{abstract}

\section{Introduction}

This article is the first of a series of three papers to come. The motivation stems from the well-known theory of Sibuya [1975] and its Gevrey-resurgent extensions, and their applications in spectral analysis.

Sibuya [1975] gives an exhaustive description of the asymptotic properties when $|x| \rightarrow \infty$ of the solutions of the ordinary differential equation $-\frac{d^{2} \Phi}{d x^{2}}+P(x) \Phi=0$, where $P(x)=x^{m}+a_{1} x^{m-1}+\cdots+a_{m}$ is a complex polynomial function of order $m$. Among various results, he shows the existence of a set of fundamental functional relations between the Stokes connection matrices, when viewed as functions of the coefficients of $P$. The asymptotic behavior of the Stokes-Sibuya coefficients when the constant term $a_{m}$ of $P$ tends to infinity is also provided. Later, these results have been clarified and extended in the framework of the Gevrey and resurgence theories [Ramis 1993; Loday-Richaud 1995; Écalle 1985; Candelpergher et al. 1993a; Voros 1983; Delabaere et al. 1993; Delabaere and Pham 1999; Costin 1998; Trinh 2005].

One of the main applications of these results is a qualitative and quantitative description of the spectral set of the Schrödinger operator $-\frac{d^{2}}{d x^{2}}+P(x)$. See, for example, [Delabaere et al. 1997; Delabaere and Pham 1997; Kawai and Takei 1994; Voros 2000]. Recently, the exact asymptotic analysis has been applied with

MSC2000: 34L40, 34M37, 34M40.

Keywords: resurgence theory, Stokes phenomena, connection problem. 
success to describe the spectral properties of a class of $P T$-symmetric oscillators, that is, when the potential function $P$ satisfies $P(x)=\overline{P(-\bar{x})}$. As a rule, such $P T$-symmetric operators are not Hermitian, therefore the existence of a real spectrum is a nonobvious but interesting question (see, for example, [Bender et al. 1999; 2002] for motivations and applications in physics). In [Delabaere and Pham 1998] and [Delabaere and Trinh 2000], the authors consider the $P T$-symmetric complex cubic oscillator, and prove the reality of the spectrum and the appearance of spontaneous symmetry breaking, this depending on the values of the coupling constant. Meanwhile, the reality of the spectrum of the $P T$-symmetric Schrödinger operator $-\frac{d^{2}}{d x^{2}}+P(x)$, with $P$ polynomial, was proved by Shin [Shin 2002] under convenient hypotheses. His work relies on a clever examination of the results of Sibuya, and on ideas and tools usually used in the context of integrable models in quantum field theory.

As a matter of fact, apart from the key-results of Sibuya, the strategy followed by Shin was previously developed by Dorey et al. [2001] to prove the reality of the spectrum of the $P T$-symmetric operator $-\frac{d^{2}}{d x^{2}}-(i x)^{2 m}-\alpha(i x)^{m-1}+l(l+1) / x^{2}$.

Our programme is to generalize all the above results to the one-dimensional Schrödinger operator $H=-\frac{d^{2}}{d x^{2}}+P(x) / x^{2}$ with $P(x)$ a complex polynomial function of order $m \in \mathbb{N}^{\star}$.

In the present article, we consider the ordinary differential equation

$\left(\mathfrak{E}_{m}\right)$

$$
\frac{d^{2}}{d x^{2}} \Phi(x)=\frac{P_{m}(x, \underline{a})}{x^{2}} \Phi(x)
$$

in the complex $x$ plane, where $\underline{a}:=\left(a_{1}, \ldots, a_{m}\right) \in \mathbb{C}^{m}, m \in \mathbb{N}^{\star}$, and

$$
P_{m}(x, \underline{a})=x^{m}+a_{1} x^{m-1}+\cdots+a_{m} \in \mathbb{C}[x] .
$$

This equation admits a unique irregular singular point located at infinity, and our aim is to concentrate on the asymptotic behaviors of the solutions of $\left(\mathfrak{E}_{m}\right)$ at this point, and their deformations in the parameter $\underline{a}=\left(a_{1}, \ldots, a_{m}\right)$. Compared with the work of Sibuya [1975], the main novelty comes from the existence (as a rule) of another singular point, a regular singular one at the origin. The Stokes-Sibuya coefficients, when considered as functions of the coefficients $\underline{a}$ of the polynomial $P_{m}$, are still governed by a set of independent functional relations, but the nontrivial monodromy at the origin has now to be taken into account. As we shall see, this translates in term of an interesting $\underline{a}$-dependent equational resurgence structure.

The paper is organized as follows. In Section 2 we study the solutions near infinity, introducing a well-behaved family of systems of fundamental solutions. The associated Stokes-Sibuya coefficients are defined, and their analytic dependence on $\underline{a}$ are analyzed. The main existence theorem given in Section 2 is proved in 
Section 3 by resurgent methods, and we compare the Stokes-Sibuya coefficients with the Stokes multipliers given by the resurgence viewpoint.

In Section 4, we introduce and study a convenient system of fundamental solutions near the origin, by means of the Fuchs theory. By comparing, in Section 5 , these different families of fundamental solutions, this yields a set of functional relations which are detailed in Section 6. Besides describing these general properties, we provide different examples in Section 7 and the Appendix, which will serve as guidelines in our next papers. In particular, for $m=3$, we provide a family of isomonodromic deformations conditions.

In a second paper, we will investigate the asymptotics of the solutions and Stokes multipliers with respect to the parameter $\underline{a}$, when $\|\underline{a}\| \rightarrow \infty$. Roughly speaking, this corresponds to the second part of [Sibuya 1975]. However, this work will be done in the framework of the exact WKB analysis, thus taking advantage of the tools and ideas of the (quantum) resurgence theory, in the spirit of [Voros 1983] and [Delabaere and Pham 1999].

A third paper will be devoted to applications to spectral analysis, especially for $P T$-symmetric operators

$$
H=-\frac{d^{2}}{d x^{2}}+\frac{P_{m}(x, \underline{a})}{x^{2}},
$$

with a generalization of the result of [Dorey et al. 2001] as an interesting byproduct.

\section{Solutions of $\left(\mathfrak{E}_{m}\right)$ in the neighbourhood of infinity: classical asymptotics}

We begin in the framework of the usual Poincaré asymptotic analysis, (see, for example, [Dieudonné 1968; Fedoryuk 1983; Olver 1974; Wasow 1965]). We are interested in the question of the existence of solutions (formal or not) at infinity for the equation $\left(\mathfrak{E}_{m}\right)$. The starting point of our study will be the main existence theorem, Theorem 2.1, which can be seen as an adaptation of a classical theorem due to Sibuya [1975, p.15]. It asserts the existence and the uniqueness of a solution of $\left(\mathfrak{E}_{m}\right)$ defined by its asymptotic expansion at infinity.

The main existence theorem. In the sequel, it will be convenient to think of $x$ as an element of the universal covering of $\mathbb{C}^{\star}$ with base point 1 . Since this covering can be identified to $\mathbb{C}$ provided with the projection $t \mapsto x=e^{t}$, we shall associated to $x$ its argument $\arg (x) \in \mathbb{R}$.

In what follows,

$$
\frac{\sqrt{P_{m}(x, \underline{a})}}{x}=x^{m / 2-1}+\sum_{k=1}^{N} b_{m / 2-k}(\underline{a}) x^{m / 2-k-1}+O\left(x^{m / 2-N}\right)
$$


stands for the asymptotic expansion at infinity in $x$ of $\sqrt{P_{m}(x, \underline{a})} / x$. Also, $x^{\alpha}=$ $\exp (\alpha \ln (x))$ with $\ln (x)$ real for $\arg (x)=0$.

Theorem 2.1. The differential equation $\left(\mathfrak{E}_{m}\right)$ admits a unique solution $\Phi_{0}(x, \underline{a})$ satisfying the following condition.

(1) $\Phi_{0}$ is an analytic function in $x$ in the sector $\Sigma_{0}=\left\{|x|>0,|\arg (x)|<\frac{3 \pi}{m}\right\}$ such that, in any strict sub-sector of $\Sigma_{0}, \Phi_{0}$ admits an asymptotic expansion at infinity of the form

$$
T \Phi_{0}(x, \underline{a})=x^{r(\underline{a})} e^{-S(x, \underline{a})} \phi_{0}(x, \underline{a}),
$$

uniformly with respect to $\underline{a}$ in any compact set of $\mathbb{C}^{m}$, where

$$
\begin{aligned}
S(x, \underline{a}) & =\frac{2}{m} x^{m / 2}+\sum_{k=1}^{(m-1) / 2} \frac{b_{m / 2-k}(\underline{a})}{m / 2-k} x^{m / 2-k} \in \mathbb{C}[\underline{a}]\left[x^{1 / 2}\right], \\
r(\underline{a}) & =\frac{1}{2}-\frac{m}{4}, \\
\phi_{0} & \in \mathbb{C}[\underline{a}] \llbracket x^{-1 / 2} \rrbracket \text { with constant term } 1,
\end{aligned}
$$

if $m$ is odd, and

$$
\begin{aligned}
S(x, \underline{a}) & =\frac{2}{m} x^{m / 2}+\sum_{k=1}^{m / 2-1} \frac{b_{m / 2-k}(\underline{a})}{m / 2-k} x^{m / 2-k} \in \mathbb{C}[\underline{a}][x], \\
r(\underline{a}) & =\frac{1}{2}-\frac{m}{4}-b_{0}(\underline{a}), \\
\phi_{0} & \in \mathbb{C}[\underline{a}] \llbracket x^{-1} \rrbracket \text { with constant term } 1,
\end{aligned}
$$

if $m$ is even.

Moreover,

(2) $\Phi_{0}$ extends analytically in $x$ to the universal covering of $\mathbb{C}^{\star}$, and is an entire function in $\underline{a}$.

(3) The derivative $\Phi_{0}^{\prime}$ of $\Phi_{0}$ with respect to $x$ admits an asymptotic expansion at infinity of the form

$$
T\left(\frac{d}{d x} \Phi_{0}(x, \underline{a})\right)=\frac{d}{d x}\left(T \Phi_{0}(x, \underline{a})\right)=x^{r(\underline{a})+m / 2-1} e^{-S(x, \underline{a})}(-1+o(1))
$$

when $x$ tends to infinity in any strict sub-sector of $\Sigma_{0}$, uniformly with respect to $\underline{a}$.

Needless to say, the asymptotic expansion $T \Phi_{0}(x, \underline{a})$ of $\Phi_{0}$ at infinity in $\Sigma_{0}$ can be computed algorithmically. For instance, for $m=3$ one gets (with Maple)

$$
\begin{aligned}
T \Phi_{0}(x, \underline{a})=e^{-\left(2 x^{3 / 2}\right) / 3-a_{1} x^{1 / 2}} x^{-1 / 4} & \left(1+\left(a_{2}-\frac{1}{4} a_{1}^{2}\right) x^{-1 / 2}\right. \\
& \left.+\left(-\frac{1}{4} a_{1}^{2} a_{2}+\frac{1}{32} a_{1}^{4}-\frac{1}{4} a_{1}+\frac{1}{2} a_{2}^{2}\right) x^{-1}+O\left(x^{-3 / 2}\right)\right),
\end{aligned}
$$


while for $m=4$,

$$
\begin{aligned}
T \Phi_{0}(x, \underline{a})= & e^{-\left(x^{2}+a_{1} x\right) / 2} x^{\left(a_{1}^{2}-4 a_{2}-4\right) / 8}\left(1+\left(\frac{1}{16} a_{1}^{3}-\frac{1}{4} a_{1} a_{2}-\frac{1}{4} a_{1}+\frac{1}{2} a_{3}\right) x^{-1}\right. \\
+ & \left(\frac{5}{32} a_{1}^{2}-\frac{1}{16} a_{2}^{2}-\frac{1}{64} a_{1}^{4} a_{2}+\frac{1}{32} a_{1}^{2} a_{2}^{2}+\frac{5}{32} a_{1}^{2} a_{2}-\frac{1}{8} a_{1} a_{2} a_{3}+\frac{1}{4} a_{4}-\frac{1}{4} a_{2}\right. \\
& \left.\left.\quad-\frac{9}{256} a_{1}^{4}-\frac{1}{4} a_{1} a_{3}-\frac{3}{16}+\frac{1}{512} a_{1}^{6}+\frac{1}{32} a_{1}^{3} a_{3}+\frac{1}{8} a_{3}^{2}\right) x^{-2}+O\left(x^{-3}\right)\right) .
\end{aligned}
$$

We shall discuss the proof of Theorem 2.1 in a moment (see Section 3). Here, we would like to show how one can derive fundamental systems of solutions of $\left(\mathfrak{E}_{m}\right)$ from $\Phi_{0}$ only. This is the subject of the following subsection.

Stokes-Sibuya coefficients. In the sequel, it will be convenient to introduce the following notations.

Notation 2.2. For all $\lambda \in \mathbb{C}$ and all $\underline{a}=\left(a_{1}, \ldots, a_{m}\right) \in \mathbb{C}^{m}$, we define

$$
\lambda . \underline{a}:=\left(\lambda a_{1}, \ldots, \lambda^{m} a_{m}\right) \text {. }
$$

We set

$$
\omega=e^{ \pm 2 i \pi / m}
$$

and introduce, for all $k \in \mathbb{Z}$,

$$
\Phi_{k}(x, \underline{a})=\Phi_{0}\left(\omega^{k} x, \omega^{k} \cdot \underline{a}\right) .
$$

where $\Phi_{0}$ is given by Theorem 2.1 .

We bring into play a quasi-homogeneity property of $\left(\mathfrak{E}_{m}\right)$. We note that $\left(\mathfrak{E}_{m}\right)$ is invariant under the transformation $(x, a) \mapsto(\omega x, \omega . \underline{a})$ so that, with the above notations, Theorem 2.1 easily translates into the following lemma.

Lemma 2.3. For any $k \in \mathbb{Z}, \Phi_{k}$ is a solution of $\left(\mathfrak{E}_{m}\right)$, and is entire in a. Its asymptotic expansion when $x$ tends to infinity in the sector

$$
\Sigma_{k}=\left\{|x|>0,|\arg (x)+k \cdot \arg (\omega)|<\frac{3 \pi}{m}\right\},
$$

uniformly in a in any compact set of $\mathbb{C}^{m}$, is given by

$$
T \Phi_{k}(x, \underline{a})=T \Phi_{0}\left(\omega^{k} x, \omega^{k} . \underline{a}\right)
$$

where $T \Phi_{0}$ is the asymptotic expansion of $\Phi_{0}$ in $\Sigma_{0}$ described in Theorem 2.1.

Corollary 2.4. For every $k \in \mathbb{Z}$, the solution $\Phi_{k}$ is exponentially decreasing ("subdominant function" in [Sibuya 1975, p19]) in the sector

$$
\Lambda_{k}=\left\{|\arg (x)+k \cdot \arg (\omega)|<\frac{\pi}{m}\right\} .
$$

We note that the sectors $\Lambda_{k-1}, \Lambda_{k}$ and $\Lambda_{k+1}$ are included in $\Sigma_{k}$ and, by Lemma 2.3, each solution $\Phi_{k}$ has an exponential growth of order not greater than $\frac{m}{2}$ in the two sectors $\Lambda_{k-1}$ and $\Lambda_{k+1}$ adjacent to $\Lambda_{k}$. This leads to the following lemma. 
Lemma 2.5. For every $k \in \mathbb{Z},\left\{\Phi_{k}, \Phi_{k+1}\right\}$ constitutes a fundamental system of solutions of $\left(\mathfrak{E}_{m}\right)$ and, moreover,

$$
W\left(\Phi_{k}, \Phi_{k+1}\right)=2(-1)^{k} \omega^{k(1-m / 2)+r\left(\omega^{k+1} . a\right)}
$$

where $W$ denotes the Wronskian, and $r$ is given by Theorem 2.1.

Proof. By quasi-homogeneity of $P_{m}(x, \underline{a})$, we note that

$$
S(\omega x, \omega \cdot \underline{a})=-S(x, \underline{a}) .
$$

Thus, by Lemma 2.3, for $x \in \Sigma_{k}$,

$$
T \Phi_{k}(x, \underline{a})=\omega^{k r\left(\omega^{k} \cdot \underline{a}\right)} x^{r\left(\omega^{k} \cdot \underline{a}\right)} e^{(-1)^{k-1} S(x, \underline{a})}(1+o(1)) .
$$

Using Theorem 2.1 part (3) we also have, for $x \in \Sigma_{k}$,

$$
T \Phi_{k}^{\prime}(x, \underline{a})=(-1)^{k-1} \omega^{k r\left(\omega^{k} \cdot \underline{a}\right)} x^{r\left(\omega^{k} \cdot \underline{a}\right)+m / 2-1} e^{(-1)^{k-1} S(x, \underline{a})}(1+o(1)) .
$$

Moreover, the coefficient $b_{0}$ of theorem 1 is a quasi-homogeneous polynomial in $\underline{a}$ of order $\frac{m}{2}$ so that

$$
r\left(\omega^{k} \cdot \underline{a}\right)+r\left(\omega^{k+1} \cdot \underline{a}\right)=1-\frac{m}{2} .
$$

As a result, we get the equalities

$$
\begin{aligned}
W\left(\Phi_{k}, \Phi_{k+1}\right) & =\Phi_{k} \Phi_{k+1}^{\prime}-\Phi_{k}^{\prime} \Phi_{k+1} \\
& =2(-1)^{k} \omega^{k\left(r\left(\omega^{k} \cdot \underline{a}\right)+r\left(\omega^{k+1} \cdot a\right)\right)+r\left(\omega^{k+1} \cdot \underline{a}\right)} x^{r\left(\omega^{k} \cdot \underline{a}\right)+r\left(\omega^{k+1} \cdot \underline{a}\right)+m / 2-1}(1+o(1)) \\
& =2(-1)^{k} \omega^{k(1-m / 2)+r\left(\omega^{k+1} \cdot \underline{a}\right)}(1+o(1)) .
\end{aligned}
$$

for $x \in \Sigma_{k} \cap \Sigma_{k+1}$. The Wronskian $W\left(\Phi_{k}, \Phi_{k+1}\right)$ being independent of $x$, this completes the proof.

Since each system $\left\{\Phi_{k}, \Phi_{k+1}\right\}$ constitutes a fundamental system of solutions of $\left(\mathfrak{E}_{m}\right)$ we deduce, from the classical theory on linear differential equations, the existence of functions $C_{k}(\underline{a})$ and $\widetilde{C}_{k}(\underline{a})$, depending only on $\underline{a}$, such that

$$
\Phi_{k-1}=C_{k}(\underline{a}) \Phi_{k}+\widetilde{C}_{k}(\underline{a}) \Phi_{k+1}
$$

for all $k \in \mathbb{Z}$.

Definition 2.6. The functions $C_{k}(\underline{a})$ and $\widetilde{C}_{k}(\underline{a})$ defined in $(2-1)$ are called the Stokes-Sibuya coefficients of $\Phi_{k-1}$ associated respectively with $\Phi_{k}$ and $\Phi_{k+1}$. The matrices

$$
\mathfrak{S}_{k}(\underline{a}):=\left(\begin{array}{cc}
C_{k}(\underline{a}) & \widetilde{C}_{k}(\underline{a}) \\
1 & 0
\end{array}\right)
$$

are called the Stokes-Sibuya connection matrices. 
Differentiating the equalities (2-1) with respect to $x$, we obtain

$$
\begin{aligned}
& C_{k}(\underline{a})=\frac{W\left(\Phi_{k-1}, \Phi_{k+1}\right)}{W\left(\Phi_{k}, \Phi_{k+1}\right)}, \\
& \widetilde{C}_{k}(\underline{a})=\frac{W\left(\Phi_{k-1}, \Phi_{k}\right)}{W\left(\Phi_{k+1}, \Phi_{k}\right)} .
\end{aligned}
$$

Using the fact that the $\Phi_{k}$ 's are entire functions in $\underline{a}$, we deduce from (2-2), (2-3) and Lemma 2.5 that the Stokes-Sibuya coefficients are entire functions in a. Also, it follows from (2-3), Lemma 2.5 and the definition of the $\Phi_{k}$ that $C_{k}(\underline{a})=C_{0}\left(\omega^{k} \cdot \underline{a}\right)$, while $\widetilde{C}_{k}(\underline{a})=\widetilde{C}_{0}\left(\omega^{k} \cdot \underline{a}\right)=\omega^{m-2+2 r\left(\omega^{k} \cdot \underline{a}\right)}$. In particular, since $\omega^{m}=e^{ \pm 2 i \pi}$, we get $C_{k}=C_{k}(\bmod m)$ and $\widetilde{\widetilde{C}}_{k}=\widetilde{C}_{k}(\bmod m)$ for all $k \in \mathbb{Z}$.

We summarize our results.

Theorem 2.7. For all $k \in \mathbb{Z}$ we denote

$$
\Phi_{k}(x, \underline{a})=\Phi_{0}\left(\omega^{k} x, \omega^{k} \cdot \underline{a}\right),
$$

where $\Phi_{0}$ is the solution of $\left(\mathfrak{E}_{m}\right)$ defined in Theorem 2.1. Then, for every $k \in \mathbb{Z}$ :

- $\Phi_{k}(x, \underline{a})$ is analytic in $x$ on the universal covering of $\mathbb{C}^{\star}$ and entire in $\underline{\text { a }}$.

- The system $\left\{\Phi_{k}, \Phi_{k+1}\right\}$ constitutes a fundamental system of solutions of $\left(\mathfrak{E}_{m}\right)$.

-We have

$$
\left(\begin{array}{c}
\Phi_{k-1} \\
\Phi_{k}
\end{array}\right)(x, \underline{a})=\mathfrak{S}_{k}(\underline{a})\left(\begin{array}{c}
\Phi_{k} \\
\Phi_{k+1}
\end{array}\right)(x, \underline{a}),
$$

where the Stokes-Sibuya connection matrix $\mathfrak{S}_{k}(\underline{a})$ is invertible, and entire in a. Moreover,

$$
\mathfrak{S}_{k}(\underline{a})=\mathfrak{S}_{k-1}(\omega \cdot \underline{a}), \quad \mathfrak{S}_{k}(\underline{a})=\mathfrak{S}_{0}\left(\omega^{k} \cdot \underline{a}\right) .
$$

- The Stokes-Sibuya coefficients $C_{k}(\underline{a})$ and $\widetilde{C}_{k}(\underline{a})$ associated with, respectively, $\Phi_{k}$ and $\Phi_{k+1}$ are entire functions in $\underline{a}$, and

$$
\begin{array}{ll}
C_{k}(\underline{a})=C_{0}\left(\omega^{k} \cdot \underline{a}\right), & C_{k}=C_{k}(\bmod m), \\
\widetilde{C}_{k}(\underline{a})=\widetilde{C}_{0}\left(\omega^{k} \cdot \underline{a}\right)=\omega^{m-2+2 r\left(\omega^{k} \cdot \underline{a}\right)}, & \widetilde{C}_{k}=\widetilde{C}_{k}(\bmod m) .
\end{array}
$$

For any $k \in \mathbb{Z}$, the analytic continuation of $\left\{\Phi_{k-1}, \Phi_{k}\right\}$ constitutes a fundamental system of solutions of $\left(\mathfrak{E}_{m}\right)$ (by Lemma 2.5, the Wronskian $W\left(\Phi_{k-1}, \Phi_{k}\right)$ does not vanish). In particular, there exists a unique invertible $2 \times 2$ matrix $\mathfrak{M}_{k}^{\infty}(\underline{a})$, entire in $\underline{a}$, such that

$$
\left(\begin{array}{c}
\Phi_{k-1} \\
\Phi_{k}
\end{array}\right)\left(\omega^{m} x, \underline{a}\right)=\mathfrak{M}_{k}^{\infty}(\underline{a})\left(\begin{array}{c}
\Phi_{k-1} \\
\Phi_{k}
\end{array}\right)(x, \underline{a}) .
$$


Definition 2.8. The $2 \times 2$ matrices $\mathfrak{M}_{k}^{\infty}(\underline{a})$, for $k \in \mathbb{Z}$, defined by

$$
\left(\begin{array}{c}
\Phi_{k-1} \\
\Phi_{k}
\end{array}\right)\left(\omega^{m} x, \underline{a}\right)=\mathfrak{M}_{k}^{\infty}(\underline{a})\left(\begin{array}{c}
\Phi_{k-1} \\
\Phi_{k}
\end{array}\right)(x, \underline{a}),
$$

are called the $\infty$-monodromy matrices.

From the definition of the $\Phi_{k}$, we note that $\mathfrak{M}_{k}^{\infty}(\underline{a})=\mathfrak{M}_{0}^{\infty}\left(\omega^{k} . \underline{a}\right)$. Also,

$$
\begin{aligned}
\left(\begin{array}{c}
\Phi_{-1} \\
\Phi_{0}
\end{array}\right)(x, \underline{a}) & =\mathfrak{S}_{0}(\underline{a}) \ldots \mathfrak{S}_{m-1}(\underline{a})\left(\begin{array}{c}
\Phi_{m-1} \\
\Phi_{m}
\end{array}\right)(x, \underline{a}) \\
& =\mathfrak{S}_{0}(\underline{a}) \ldots \mathfrak{S}_{m-1}(\underline{a})\left(\begin{array}{c}
\Phi_{-1} \\
\Phi_{0}
\end{array}\right)\left(\omega^{m} x, \omega^{m} \cdot \underline{a}\right) .
\end{aligned}
$$

Since $\left(\begin{array}{c}\Phi_{-1} \\ \Phi_{0}\end{array}\right)$ is entire in $\underline{a}$, we obtain

$$
\begin{aligned}
\left(\begin{array}{c}
\Phi_{-1} \\
\Phi_{0}
\end{array}\right)(x, \underline{a}) & =\mathfrak{S}_{0}(\underline{a}) \ldots \mathfrak{S}_{m-1}(\underline{a})\left(\begin{array}{c}
\Phi_{-1} \\
\Phi_{0}
\end{array}\right)\left(\omega^{m} x, \underline{a}\right) \\
& =\mathfrak{S}_{0}(\underline{a}) \ldots \mathfrak{S}_{m-1}(\underline{a}) \mathfrak{M}_{0}^{\infty}(\underline{a})\left(\begin{array}{c}
\Phi_{-1} \\
\Phi_{0}
\end{array}\right)(x, \underline{a}),
\end{aligned}
$$

and, $\left\{\Phi_{-1}, \Phi_{0}\right\}$ being a fundamental system, this yields the following theorem.

Theorem 2.9. For every $k \in \mathbb{Z}$, the $\infty$-monodromy matrix $\mathfrak{M}_{k}^{\infty}(\underline{a})$ is invertible, entire in $\underline{a}$, and

$$
\mathfrak{M}_{k}^{\infty}(\underline{a})=\mathfrak{M}_{0}^{\infty}\left(\omega^{k} \cdot \underline{a}\right)
$$

Furthermore, the Stokes-Sibuya matrices satisfy the functional relation

$$
\mathfrak{S}_{0}(\underline{a}) \ldots \mathfrak{S}_{m-1}(\underline{a})=\left(\mathfrak{M}_{0}^{\infty}(\underline{a})\right)^{-1} .
$$

Relation (2-10) generalizes a functional relation due to Sibuya [1975, p85]. Unfortunately, as a rule, the $\infty$ - monodromy matrix $\mathfrak{M}_{0}^{\infty}$ is difficult to compute. We return to this question in Section 6.

\section{Solutions of $\left(\mathfrak{E}_{m}\right)$ in the neighbourhood of infinity: resurgent point of view}

Theorem 2.1 can be shown with the methods developed in Sibuya's book [1975] and, in fact, is actually proved in [Mullin 1968; Bakken 1977]. However, using the resurgent viewpoint, one can get a stronger result in a simpler way. 
Basic notions in resurgence theory. Since the terminology we use in this section is likely to be least familiar to some readers, we first give the necessary definitions; see [Candelpergher et al. 1993b; Candelpergher et al. 1993a; Delabaere and Pham 1997; Écalle 1981] for more details. We mention that our notation differs from that usually used by Écalle. As usual in this article, we identify an element of the universal covering of $\mathbb{C}^{\star}$ (with base point 1 ) by specifying its argument in $\mathbb{R}$.

Definition 3.1. A sectorial neighbourhood of infinity of aperture $I=] \alpha, \beta[\subset \mathbb{R}$ is an open set $U$ of the universal covering of $\mathbb{C}^{\star}$ such that for any open interval $J \subset I$, there is $z \in U$ such that $z J \subset U$, where

$$
z J:=\left\{z+r e^{i \theta}: r>0, \theta \in J\right\} .
$$

Definition 3.2. If $U$ is a sectorial neighbourhood of infinity of aperture $I$ and if $\Psi$ is holomorphic in $U$, then $\Psi$ is of exponential growth of order 1 at infinity in $U$ if for any open interval $J \subset I$, there exist $\tau>0$ and $C>0$ such that

$$
|\Psi(z)| \leq C e^{\tau|z|}
$$

for all $z \in U \cap 0 J$.

We now introduce the notion of a minor. We do this only for a particular class of formal power series which will be used in this paper.

Definition 3.3. Consider the formal power series

$$
\psi(z)=r+\sum_{n=0}^{\infty} \frac{\alpha_{n}}{z^{1+n / m}} \in \mathbb{C} \llbracket z^{-1 / m} \rrbracket,
$$

where $m$ is a positive integer. Then $r$ is the residual coefficient of $\psi$, and

$$
\widetilde{\psi}(\zeta)=\sum_{n=0}^{\infty} \frac{\alpha_{n}}{\Gamma(1+n / m)} \zeta^{\frac{n}{m}} \in \mathbb{C} \llbracket \zeta^{1 / m} \rrbracket
$$

is the minor of $\psi$.

In other words, the minor of the formal power series $\psi$ is just the Borel transform without its residual coefficient. This allows to define the Borel-summability for such formal series.

Definition 3.4. The formal power series

$$
\psi(z)=r+\sum_{n=0}^{\infty} \frac{\alpha_{n}}{z^{1+n / m}} \in \mathbb{C} \llbracket z^{-1 / m} \rrbracket
$$

is Borel-resummable in the direction (of the argument) $\alpha \in \mathbb{R}$ if 
(1) its minor $\widetilde{\psi}(\zeta)$ defines a (ramified) analytic function at the origin (for simplicity, we keep the same notation $\widetilde{\psi}(\zeta)$ for the series and its sum, and $\zeta$ should be regarded as an element of the universal covering of $\left.\mathbb{C}^{\star}\right)$, and

(2) there exists an open sector $0 I$ with $I$ an open neighbourhood of $\alpha$ such that $\widetilde{\psi}(\zeta)$ can be analytically extended in $0 I$ and is of exponential growth of order 1 at infinity in $0 I$.

The Borel sum $\mathrm{S}_{\alpha} \psi(z)$ with respect to $z$, in the direction $\alpha \in \mathbb{R}$, of the formal series $\psi$ is defined by

$$
\mathrm{S}_{\alpha} \psi(z):=r+\int_{0}^{\infty e^{i \alpha}} e^{-z \zeta} \widetilde{\psi}(\zeta) d \zeta .
$$

In Definition 3.4, when one drops the growth condition (2) at infinity, $\psi$ is said to be Borel presummable in the direction $\alpha$, the summation operator $\mathrm{s}_{\alpha}$ being replaced by the presummation operator which we do not define here (see, for example, [Delabaere and Pham 1997]).

A Borel sum has the following main properties.

Proposition 3.5. If

$$
\psi(z)=r+\sum_{n=0}^{\infty} \frac{\alpha_{n}}{z^{1+n / m}} \in \mathbb{C} \llbracket z^{-1 / m} \rrbracket
$$

is Borel-resummable in the direction $\alpha \in \mathbb{R}$, then

(1) its Borel sum $\mathrm{S}_{\alpha} \psi(z)$ is holomorphic in a sectorial neighbourhood of infinity $U$ of aperture $I=]-\frac{\pi}{2}-\alpha, \frac{\pi}{2}-\alpha[$,

(2) $\mathrm{S}_{\alpha} \psi(z)$ is asymptotic to $\psi(z)$ at infinity in $U$. More precisely, for any strict subinterval $J$ of $I$, there is $C>0$ such that

$$
\left|\mathrm{S}_{\alpha} \psi(z)-r-\sum_{k=0}^{n-1} \frac{\alpha_{k}}{z^{1+k / m}}\right| \leq C^{n} \Gamma(1+n / m)|z|^{-n / m-1} .
$$

for all $n \geq 1$, and all $z \in U \cap 0 J$,

(3) $\frac{d}{d z}\left(\mathrm{~S}_{\alpha} \psi(z)\right)=\mathrm{S}_{\alpha}\left(\frac{d \psi}{d z}(z)\right)$.

(4) If two formal power series $\psi(z), \phi(z) \in \mathbb{C} \llbracket z^{-1 / m} \rrbracket$ are Borel-resummable in the direction $\alpha \in \mathbb{R}$, then $\mathrm{S}_{\alpha}(\psi \cdot \phi)(z)=\mathrm{S}_{\alpha}(\psi)(z) . \mathrm{S}_{\alpha}(\phi)(z)$.

Definition 3.6. A formal power series

$$
\psi(z)=r+\sum_{n=0}^{\infty} \frac{\alpha_{n}}{z^{1+n / m}} \in \mathbb{C} \llbracket z^{-1 / m} \rrbracket
$$

is resurgent if its minor $\widetilde{\psi}(\zeta)$ defines a (ramified) analytic function at the origin, and is endlessly continuable, that is, for every $L>0$ there is a finite subset $\Omega_{L} \subset \mathbb{C}$ 
such that $\widetilde{\psi}$ can be analytically continued along every path $\lambda$ of length $<L$ which avoids $\Omega_{L}$.

This definition can be extended to an algebra of resurgent formal functions which we do not define precisely here. (Note that Écalle proposes a more general definition.)

\section{Proposition 3.7. If two formal power series}

$$
\psi(z)=r+\sum_{n=0}^{\infty} \frac{\alpha_{n}}{z^{1+n / m}} \in \mathbb{C} \llbracket z^{-1 / m} \rrbracket \quad \text { and } \quad \phi(z)=s+\sum_{n=0}^{\infty} \frac{\beta_{n}}{z^{1+n / m}} \in \mathbb{C} \llbracket z^{-1 / m} \rrbracket
$$

are resurgent, then the product $\psi . \phi(z)$ is too: the minor of $\psi . \phi(z)$ given by

$$
\begin{aligned}
\widetilde{\psi} \cdot \phi(\zeta) & =r \cdot \tilde{\phi}(\zeta)+s \cdot \widetilde{\psi}(\zeta)+\widetilde{\psi} * \widetilde{\phi}(\zeta), \\
\widetilde{\psi} * \widetilde{\phi}(\zeta) & =\int_{0}^{\zeta} \widetilde{\psi}(\eta) \widetilde{\phi}(\zeta-\eta) d \eta
\end{aligned}
$$

(where the latter is the convolution product) is endlessly continuable.

For a resurgent formal power series, it may happen that we no longer can define its Borel (pre)sum in a given direction $\alpha \in \mathbb{R}$ because the minor can have singularities along this direction: this is the essence of the Stokes phenomenon.

Definition 3.8. We consider a resurgent formal power series

$$
\psi(z)=r+\sum_{n=0}^{\infty} \frac{\alpha_{n}}{z^{1+n / m}} \in \mathbb{C} \llbracket z^{-1 / m} \rrbracket .
$$

Let $\alpha \in \mathbb{R}$ be a singular direction for the minor $\widetilde{\psi}(\zeta)$. We hypothesise that there is an $\epsilon>0$ such that $\widetilde{\psi}(\zeta)$ can be analytically extended in the open sector 0$] \alpha, \alpha+\epsilon[$ (respectively, 0$] \alpha-\epsilon, \alpha$ ) with an exponential growth of order 1 at infinity. We assume also that this exponential growth at infinity extends up to paths $\left[0, \infty e^{i \alpha}+[\right.$ and $\left[0, \infty e^{i \alpha}-[)\right.$ circumventing the singularities to the left and right, respectively, along the direction $\alpha$ :

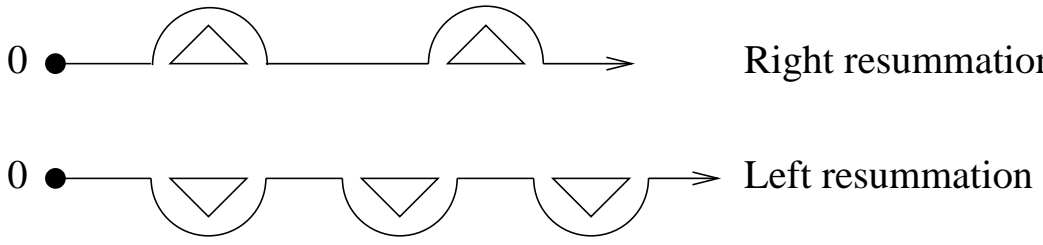

Then $\psi$ is right (respectively, left) Borel-resummable in the direction $\alpha$, its right (respectively, left) Borel sum $\mathrm{S}_{\alpha+} \psi$ (respectively, $\mathrm{S}_{\alpha-} \psi$ ) being defined by

$$
\mathrm{S}_{\alpha \pm} \psi(z):=r+\int_{0}^{\infty e^{i \alpha} \pm} e^{-z \zeta} \widetilde{\psi}(\zeta) d \zeta
$$


for $z$ in a sectorial neighbourhood of infinity of aperture $]-\frac{\pi}{2}-\alpha, \frac{\pi}{2}-\alpha[$.

In Definition 3.8, it is possible to drop the hypothesis, replacing the right (respectively, left) Borel sum by the right (respectively, left) Borel presum (see, for example, [Delabaere and Pham 1997]). In other words, every resurgent formal function is always right and left Borel-presummable (in any direction).

We note that Proposition 3.5 is still valid for right and left Borel sum. Moreover, when $\psi$ is Borel (pre)summable in the direction $\alpha \in \mathbb{R}$, then

$$
\mathrm{S}_{\alpha} \psi(z)=\mathrm{S}_{\alpha+} \psi(z)=\mathrm{S}_{\alpha-} \psi(z) .
$$

In order to be able to compare right and left (pre)summation, one has to enlarge the set of resurgent formal functions to the set of resurgent symbols.

Definition 3.9. A resurgent symbol (or resurgent transseries) in the direction $\alpha$ is a formal sum

$$
\varphi(z)=\sum_{\omega \in \Omega} \psi_{\omega} e^{-z \omega}
$$

where each $\psi_{\omega}(z)$ is a resurgent formal function and $\Omega$, the singular support of $\varphi$, is a discrete subset of $\left[0, \infty e^{i \alpha}[\right.$.

The sum and product of two resurgent symbols are defined in an obvious fashion, so that resurgent symbols in the direction $\alpha$ make up an algebra which we denote by $\mathscr{R}_{\alpha}$.

The right and left (pre)summation operations can be extended to resurgent symbols in a way so that

$$
\mathrm{S}_{\alpha+} \varphi=\sum_{\omega \in \Omega}\left(\mathrm{s}_{\alpha+} \psi_{\omega}\right) e^{-z \omega} \quad \text { and } \quad \mathrm{S}_{\alpha-} \varphi=\sum_{\omega \in \Omega}\left(\mathrm{s}_{\alpha-} \psi_{\omega}\right) e^{-z \omega} .
$$

The construction (which we do not explain here) makes the operations $\mathrm{S}_{\alpha+}$ and $\mathrm{S}_{\alpha-}$ isomorphisms of algebras and, moreover, $\mathrm{S}_{\alpha+}\left(\mathscr{R}_{\alpha}\right)=\mathrm{S}_{\alpha-}\left(\mathscr{R}_{\alpha}\right)$. This key result (due to Écalle) allows to define the so-called Stokes automorphism, which analyzes the Stokes phenomenon by comparing right and left Borel (pre)summations.

Definition 3.10. The Stokes automorphism in the direction $\alpha$ is defined by

$$
\mathfrak{S}_{\alpha}:=\mathrm{S}_{\alpha+}^{-1} \circ \mathrm{S}_{\alpha-}: \mathscr{R}_{\alpha} \rightarrow \mathscr{R}_{\alpha} .
$$

The action of the Stokes automorphism in a given direction can be understood in terms of deformation of the contour of integration in a Laplace integral:

0

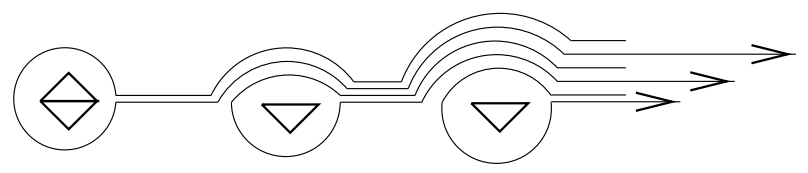


It follows from the definitions that the Stokes automorphism in the direction $\alpha$ acts trivially on exponentials $e^{-z \omega}$, and that its action on a formal resurgent series $\psi$ is given by

$$
\mathfrak{S}_{\alpha} \psi=\psi+\sum_{\omega} \psi_{\omega} e^{-z \omega},
$$

where the sum runs over those singular points of the minor $\widetilde{\psi}$ which have to be avoided when considering left (pre)summation. The Stokes automorphism $\mathfrak{S}_{\alpha}$ is given by

$$
\mathfrak{S}_{\alpha}=\mathbf{1}+{ }^{+} \boldsymbol{S}_{\alpha}
$$

where the operator ${ }^{+} \boldsymbol{S}_{\alpha}$ commutes with multiplication by exponentials, and transforms formal resurgent series into "exponentially small resurgent symbols". This implies that the operator

$$
\dot{\dot{\Delta}}_{\alpha}:=\ln \mathfrak{S}_{\alpha}=\sum_{n=1}^{\infty} \frac{(-1)^{n-1}}{n}+\boldsymbol{S}_{\alpha}^{n}
$$

is well defined on $\mathscr{R}_{\alpha}$. Since $\mathfrak{S}_{\alpha}$ is an automorphism of $\mathscr{R}_{\alpha}$, it follows that $\dot{\underline{\Delta}}_{\alpha}$ is a derivation of $\mathscr{R}_{\alpha}$.

Definition 3.11. The operator $\underline{\dot{\Delta}}_{\alpha}$ is called the alien derivation in the direction $\alpha$.

We note that since $\mathrm{S}_{\alpha+}$ and $\mathrm{S}_{\alpha-}$ commute with $\frac{d}{d z}$, it follows that $\mathfrak{S}_{\alpha}$ and $\dot{\dot{\Delta}}_{\alpha}$ also commute with $\frac{d}{d z}$.

Proposition 3.12. The alien derivation $\dot{\underline{\Delta}}_{\alpha}$ commutes with $\frac{d}{d z}$.

The alien derivation $\dot{\Delta}_{\alpha}$ commutes with multiplication by exponentials, and its action on a formal resurgent series $\psi$ has the explicit form

$$
\dot{\underline{\dot{b}}}_{\alpha} \psi=\sum_{\omega \in \Omega(\widetilde{\psi})} e^{-z \omega} \Delta_{\omega} \psi
$$

where $\Omega(\widetilde{\psi})$ is a discrete subset of $\left[0, \infty e^{i \alpha}[\right.$, the set of so-called glimpsed singularities of $\widetilde{\psi}$, that is, the set of singularities to be circumvented when analytically continuing $\widetilde{\psi}$ in the direction $\alpha$. Since $\dot{\Delta}_{\alpha}$ is a derivation, each $\Delta_{\omega}$ is also a derivation.

Definition 3.13. The derivation $\Delta_{\omega}$ is called the alien derivation at $\omega$.

Definition 3.14. A formal resurgent function $\psi$ is a constant of resurgence if $\Delta_{\omega} \psi=0$ for any $\omega$.

For instance, a convergent power series $\psi$ is a constant of resurgence. 
Instead of working with the $\Delta_{\omega}$, it is sometimes convenient to work with the so-called pointed alien derivations

$$
\dot{\Delta}_{\omega}:=e^{-z \omega} \Delta_{\omega},
$$

which have the advantage of commuting with $\frac{d}{d z}$ (this is a consequence of Proposition 3.12).

Proposition 3.15. The pointed alien derivations $\dot{\Delta}_{\omega}$ commute with $\frac{d}{d z}$.

Resurgence of solutions of $\left(\mathfrak{E}_{m}\right)$. We now return to the proof of Theorem 2.1. The main idea will be to consider the asymptotic expansion $T \Phi_{0}$ of Theorem 2.1 as a formal solution of $\left(\mathfrak{E}_{m}\right)$, and to show that $\Phi_{0}$ can be constructed from $T \Phi_{0}$ by Borel resummation with respect to an appropriate variable $z=z(x, \underline{a})$ (or $\tilde{z}$, depending on the parity of $m$ ) which will be defined later, uniformly in $\underline{a}$, for $\underline{a}$ in any given compact set.

We start with a kind of preparation theorem, so as to transform equation $\left(\mathfrak{E}_{m}\right)$ into a normal form. This is based on the Green-Liouville transformation, but we shall have to dissociate the $m$ odd case from the $m$ even case for technical reasons.

In what follows, $\underline{a}$ is assumed to belong to an arbitrary compact set $K \subset \mathbb{C}^{m}$.

Case 1: $m$ odd. We consider the Green-Liouville transformation

$$
\begin{aligned}
z= & z(x, \underline{a})=\int^{x} \frac{\sqrt{P_{m}(t, \underline{a})}}{t} d t, \\
\Psi(z, \underline{a}) & =\frac{P_{m}(x, \underline{a})^{1 / 4}}{\sqrt{x}} \Phi(x, \underline{a}),
\end{aligned}
$$

where the Laurent-Puiseux series expansion in $x$ of $z(x, \underline{a})$,

$$
z(x, \underline{a})=\frac{2}{m} x^{m / 2}+\sum_{k=1}^{(m-1) / 2} \frac{b_{m / 2-k}(\underline{a})}{m / 2-k} x^{m / 2-k}+O\left(x^{-1 / 2}\right) \in x^{m / 2} \mathbb{C}[\underline{a}]\left\{x^{-1}\right\},
$$

coincides with the map $x \mapsto S(x, \underline{a})$ defined in Theorem 2.1 modulo an analytic (multivalued) function vanishing at infinity. We note that, if $X \stackrel{\pi}{\longrightarrow} \mathbb{C}$ (respectively, $Z \stackrel{\widetilde{\pi}}{\longrightarrow} \mathbb{C}$ ) is the (ramified) Riemann surface of $x^{1 / 2}$ (respectively, $z^{1 / m}$ ), then there exists a compact set $B$ (depending on $K$ ) (respectively, $\widetilde{B}$ ) such that the map

$$
(x, \underline{a}) \in \pi^{-1}(\mathbb{C} \backslash B) \times K \mapsto(z(x, \underline{a}), \underline{a}) \in \tilde{\pi}^{-1}(\mathbb{C} \backslash \widetilde{B}) \times K
$$

is biholomorphic.

We remark here that by the quasi-homogeneity of $P_{m}(x, \underline{a})$, it follows that

$$
z(\omega x, \omega . \underline{a})=\omega^{m / 2} z(x, \underline{a}) .
$$


The transformation (3-2) converts $\left(\mathfrak{E}_{m}\right)$ into the equation

$$
-\frac{d^{2}}{d z^{2}} \Psi+(1-F(z, \underline{a})) \Psi=0
$$

which is our prepared normal form.

It is straightforward to see that studying $\left(\mathfrak{E}_{m}\right)$ at infinity in the variable $x$ amounts to studying (3-4) at infinity in the variable $z$. The inverse map $x:(z, \underline{a}) \mapsto x(z, \underline{a})$ can be identified with its Laurent-Puiseux series expansion

$$
x(z, \underline{a})=\left(\frac{m}{2} z\right)^{2 / m}+O(1) \in z^{2 / m} \mathbb{C}[\underline{a}]\left\{z^{-2 / m}\right\},
$$

and, from (3-3),

$$
x\left(\omega^{\frac{m}{2}} z, \omega \cdot \underline{a}\right)=\omega x(z, \underline{a}) .
$$

In (3-4), $F(z, \underline{a})$ is defined by

$$
F(z, \underline{a})=-\frac{x P_{m}^{\prime}(x, \underline{a})+P_{m}(x, \underline{a})}{4 P_{m}(x, \underline{a})^{2}}-\left.\frac{x^{2}}{4}\left(\frac{P_{m}^{\prime \prime}(x, \underline{a})}{P_{m}(x, \underline{a})^{2}}-\frac{5}{4} \frac{P_{m}^{\prime}(x, \underline{a})^{2}}{P_{m}(x, \underline{a})^{3}}\right)\right|_{x=x(z, \underline{a}) .}
$$

One infers from (3-5) that

$$
F(z, \underline{a})=\frac{m^{2}-4}{4 m^{2} z^{2}}\left(1+O\left(z^{-2 / m}\right)\right) \in \frac{1}{z^{2}} \mathbb{C}[\underline{a}]\left\{z^{-2 / m}\right\}
$$

is an analytic function at infinity in $z^{-1 / m}$, uniformly in $\underline{a} \in K$. It is easy to show the existence of a unique formal solution $\Psi_{+}(z, \underline{a})$ of (3-4) satisfying

$$
\Psi_{+}(z, \underline{a})=e^{-z} \psi_{+}(z, \underline{a}),
$$

where

$$
\psi_{+}(z, \underline{a})=1+\sum_{n=0}^{\infty} \frac{\alpha_{n}(\underline{a})}{z^{1+n / m}} \in \mathbb{C}[\underline{a}] \llbracket z^{-1 / m} \rrbracket
$$

with 1 for its residual coefficient. Moreover the formal power series expansion $\psi_{+}(z, \underline{a})$ satisfies the ordinary differential equation

$$
-\frac{d^{2}}{d z^{2}} \psi_{+}+2 \frac{d}{d z} \psi_{+}-F(z, \underline{a}) \psi_{+}=0
$$

In addition, by the quasi-homogeneity property of $P_{m}$, from (3-6) and (3-7), one sees that

$$
F\left(\omega^{m / 2} z, \omega . \underline{a}\right)=F(z, \underline{a}) .
$$




\section{Defining}

$$
\psi_{-}(z, \underline{a})=\psi_{+}\left(\omega^{m / 2} z, \omega . \underline{a}\right),
$$

one deduces the existence of a unique formal solution $\Psi_{-}(z, \underline{a})$ of (3-4) such that $\Psi_{-}(z, \underline{a})=e^{z} \psi_{-}(z, \underline{a})$ for $\psi_{-} \in \mathbb{C}[\underline{a}] \llbracket z^{-1 / m} \rrbracket$, with a residual coefficient equal to 1 . Note that $\Psi_{+}$and $\Psi_{-}$are linearly independent, so $\left(\Psi_{+}, \Psi_{-}\right)$provides a fundamental system of formal solutions for the linear second order equation (3-4).

The formal series $\psi_{ \pm}$enjoys the following properties.

Proposition 3.16. The formal power series expansion

$$
\psi_{+}(z, \underline{a})=1+\sum_{n=0}^{\infty} \frac{\alpha_{n}(\underline{a})}{z^{1+n / m}} \in \mathbb{C}[\underline{a}] \llbracket z^{-1 / m} \rrbracket
$$

(respectively, $\left.\psi_{-}(z, \underline{a})=\psi_{+}\left(\omega^{m / 2} z, \omega . \underline{a}\right)\right)$ is Borel-resummable with respect to $z$, uniformly in $\underline{a}$, for $\underline{a}$ in any compact set of $\mathbb{C}^{m}$, for every direction of summation except those of argument $\pi(\bmod 2 \pi)($ respectively, $0(\bmod 2 \pi))$.

Proof. We have to analyze the analytic properties of the minor

$$
\widetilde{\psi}_{+}(\zeta, \underline{a})=\sum_{n=0}^{\infty} \frac{\alpha_{n}(\underline{a})}{\Gamma\left(\frac{n}{m}+1\right)} \zeta^{n / m} \in \mathbb{C}[\underline{a}] \llbracket \zeta^{1 / m} \rrbracket .
$$

of $\psi_{+}(z, \underline{a})$. To proceed, we go back to equation (3-11). Instead of considering this differential equation, we shall rather introduce its deformation

$$
-\frac{d^{2}}{d z^{2}} \psi+2 \frac{d}{d z} \psi-F(z, \underline{a})+\varepsilon F(z, \underline{a})(1-\psi)=0,
$$

where $\varepsilon$ can be thought of as a parameter of perturbation. The introduction of this parameter will help us to rewrite $\psi_{+}$and its minor $\widetilde{\psi}_{+}$into an analyzable form, since (3-15) reduces to (3-11) when $\varepsilon=1$. We now look for a formal solution of (3-15) in the form of a normalized series expansion with respect to $\varepsilon$ :

$$
\psi(z, \underline{a}, \varepsilon)=1+\sum_{n=0}^{\infty} \psi_{n}(z, \underline{a}) \varepsilon^{n}, \quad \psi_{n} \in \frac{1}{z} \mathbb{C}[\underline{a}] \llbracket z^{-1 / m} \rrbracket .
$$

Plugging (3-16) into (3-15) and identifying the powers of $\varepsilon$, one gets

$$
\begin{aligned}
& -\frac{d^{2}}{d z^{2}} \psi_{0}+2 \frac{d}{d z} \psi_{0}=F(z, \underline{a}), \\
& -\frac{d^{2}}{d z^{2}} \psi_{n+1}+2 \frac{d}{d z} \psi_{n+1}=F(z, \underline{a}) \psi_{n}, \quad \text { for } n \geq 0 .
\end{aligned}
$$


This translates into the fact that the minors $\widetilde{\psi}_{n}(\zeta, \underline{a})$ of the $\psi_{n}(z, \underline{a})$ have to satisfy the convolution equations

$$
\begin{aligned}
& -\zeta(2+\zeta) \widetilde{\psi}_{0}=\widetilde{F}, \\
& -\zeta(\zeta+2) \widetilde{\psi}_{n+1}=\widetilde{\psi}_{n} * \widetilde{F}, \quad \text { for } n \geq 0,
\end{aligned}
$$

where $\widetilde{F}(\zeta, \underline{a})$ is the minor of $F(z, \underline{a})$, while $*$ stands for the convolution product (see (3-1)).

We have now to study the analytic properties of

$$
\widetilde{\psi}(\zeta, \underline{a}, \varepsilon)=\sum_{n=0}^{\infty} \widetilde{\psi}_{n}(\zeta, \underline{a}) \varepsilon^{n} .
$$

The key-point of the analysis will come from the properties of $F$. Writing

$$
F(z, \underline{a})=\sum_{n=0}^{\infty} \frac{f_{n}(\underline{a})}{z^{2+2 n / m}} \in \frac{1}{z^{2}} \mathbb{C}[\underline{a}]\left\{z^{-2 / m}\right\},
$$

we know that

$$
G(z)=\sum_{n=0}^{\infty} \frac{g_{n}}{z^{2+2 n / m}} \quad \text { with } \quad g_{n}=\sup _{\underline{a} \in K}\left|f_{n}(\underline{a})\right|,
$$

is an analytic function at infinity in $z^{-1 / m}$. Therefore its minor

$$
\widetilde{G}(\zeta)=\sum_{n=0}^{\infty} \frac{g_{n}}{\Gamma(2+2 n / m)} \zeta^{1+2 n / m} \in \zeta \mathbb{C}\left\{\zeta^{2 / m}\right\}
$$

is an entire function in $\zeta^{1 / m}$ (with an exponential growth at infinity of order at most 1). Thus, if $\mathfrak{C}_{m}$ denotes the Riemann surface of $\zeta^{1 / m}$, then

$$
\widetilde{F}(\zeta, \underline{a})=\sum_{n=0}^{\infty} \frac{f_{n}(\underline{a})}{\Gamma(2+2 n / m)} \zeta^{1+2 n / m} \in \zeta \mathbb{C}[\underline{a}]\left\{\zeta^{2 / m}\right\}
$$

is a holomorphic function in $(\zeta, \underline{a}) \in \mathfrak{C}_{m} \times K$ such that

$$
|\widetilde{F}(\zeta, \underline{a})| \leq G(|\zeta|) \quad \text { for all }(\zeta, \underline{a}) \in \mathfrak{C}_{m} \times K .
$$

Using the fact that $\widetilde{F}$ is a holomorphic function in $(\zeta, \underline{a}) \in \mathfrak{C}_{m} \times K$ such that $F(\zeta, \underline{a})=O(\zeta)$ uniformly in $\underline{a} \in K$, and from the properties of the convolution product, one easily deduces from $(3-18)$ that each $\widetilde{\psi}_{n}$ belongs to the space $\mathbb{C}[\underline{a}]\left\{\zeta^{1 / m}\right\}$ and extends analytically to $\widetilde{\mathbb{C}} \backslash\{0,-2\} \times K$, where $\widetilde{\mathbb{C}} \backslash\{0,-2\}$ denotes the universal covering of $\mathbb{C} \backslash\{0,-2\}$. For $\rho>0$, we now define the star-shape domain

$$
\Omega_{m}(\rho)=\left\{\zeta \in \mathfrak{C}_{m},|\dot{\zeta}+2|>\rho,[0, \zeta] \in \Omega_{m}(\rho)\right\} \subset \mathfrak{C}_{m}
$$


where $\dot{\zeta}$ is the projection of $\zeta$ by the natural mapping $\mathfrak{C}_{m} \rightarrow \mathbb{C}$. We also introduce the sequence of analytic functions $h_{n}(\zeta)$ defined for $\zeta \in \mathfrak{C}_{m}$ by

$$
\begin{aligned}
\zeta \rho \widetilde{h}_{0} & =\widetilde{G}, \\
\zeta \rho \tilde{h}_{n+1} & =\tilde{h}_{n} * \widetilde{G} \quad \text { for } n \geq 0
\end{aligned}
$$

Comparing (3-25) with (3-18), and using (3-23), one gets

$$
\left|\tilde{\psi}_{n}(\zeta, \underline{a})\right| \leq \tilde{h}_{n}(|\zeta|) \quad \text { for all }(\zeta, \underline{a}) \in \Omega_{m}(\rho) \times K \text { and all } n \in \mathbb{N} \text {. }
$$

This can be shown by an easy recursion. We just detail here the $n=0$ and $n=1$ cases. For all $(\zeta, \underline{a}) \in\left(\Omega_{m}(\rho) \backslash\{0\}\right) \times K$ we have

$$
\left|\widetilde{\psi}_{0}(\zeta, \underline{a})\right|=\frac{|\widetilde{F}(\zeta, \underline{a})|}{|\zeta||\zeta+2|} \leq \frac{\widetilde{G}(|\zeta|)}{|\zeta| \rho}=\tilde{h}_{0}(|\zeta|),
$$

and this inequality extends to $\zeta=0$ by continuity. This proves (3-26) for $n=0$. We thus deduce that, for all $(\zeta, \underline{a}) \in\left(\Omega_{m}(\rho) \backslash\{0\}\right) \times K$,

$$
\left|\widetilde{\psi}_{1}(\zeta, \underline{a})\right|=\frac{\left|\widetilde{F} * \widetilde{\psi}_{0}(\zeta, \underline{a})\right|}{|\zeta||\zeta+2|} \leq \frac{\left|\int_{0}^{\zeta} \widetilde{F}(\eta, \underline{a}) \widetilde{\psi}_{0}(\zeta-\eta, \underline{a}) d \eta\right|}{|\zeta| \rho} .
$$

Writing $\zeta=|\zeta| e^{i \theta}$ and making the change of variable $\eta=t e^{i \theta}$, we get

$$
\begin{aligned}
\left|\int_{0}^{\zeta} \widetilde{F}(\eta, \underline{a}) \widetilde{\psi}_{0}(\zeta-\eta, \underline{a}) d \eta\right| & =\left|\int_{0}^{|\zeta|} \widetilde{F}\left(t e^{i \theta}, \underline{a}\right) \widetilde{\psi}_{0}\left((|\zeta|-t) e^{i \theta}, \underline{a}\right) d t\right| \\
& \leq \int_{0}^{|\zeta|}\left|\widetilde{F}\left(t e^{i \theta}, \underline{a}\right)\right| \cdot\left|\widetilde{\psi}_{0}\left((|\zeta|-t) e^{i \theta}, \underline{a}\right)\right| d t \\
& \leq \int_{0}^{|\zeta|} \widetilde{G}(t) \tilde{h}_{0}(|\zeta|-t) d t \\
& =\widetilde{G} * \tilde{h}_{0}(|\zeta|) .
\end{aligned}
$$

Therefore, for all $(\zeta, \underline{a}) \in\left(\Omega_{m}(\rho) \backslash\{0\}\right) \times K$,

$$
\left|\widetilde{\psi}_{1}(\zeta, \underline{a})\right| \leq \frac{\widetilde{G} * \tilde{h}_{0}(|\zeta|)}{|\zeta| \rho}=\tilde{h}_{1}(|\zeta|)
$$

This gives (3-26) for $n=1$ by a continuity argument. Now $\tilde{h}(\zeta, \varepsilon)=\sum_{n=0}^{\infty} \tilde{h}_{n}(\zeta) \varepsilon^{n}$ is nothing but the minor of the series expansion $h(z, \varepsilon)=\sum_{n=0}^{\infty} h_{n}(z) \varepsilon^{n}$, where the $h_{n}$ are defined recursively by

$$
\begin{aligned}
-\rho \frac{d}{d z} h_{0} & =G, \\
-\rho \frac{d}{d z} h_{n+1} & =h_{n} G \quad \text { for } n \geq 0 .
\end{aligned}
$$


This means that $h$ satisfies the ordinary differential equation

$$
-\rho \frac{d}{d z} h=\varepsilon G(z) h+G(z) .
$$

From (3-21), we see that $G$ is integrable at infinity, so that the function

$$
(z, \varepsilon) \mapsto\left(e^{-(\varepsilon / \rho) \int_{+\infty}^{z} G\left(z^{\prime}\right) d z^{\prime}}-1\right) / \varepsilon
$$

is a solution of (3-28) which is holomorphic for $z$ in a neighbourhood of infinity of $\mathfrak{C}_{m}$ and $\varepsilon \in D(0, R), R>1$. Moreover, its Taylor series expansion at $\varepsilon=0$ is exactly $h(z, \varepsilon)=\sum_{n=0}^{\infty} h_{n}(z) \varepsilon^{n}$. In return, this proves that $\tilde{h}(\zeta, \varepsilon)$ defines a holomorphic function in $(\zeta, \varepsilon) \in \mathfrak{C}_{m} \times D(0, R)$, with an exponential growth of order not greater than 1 at infinity in $\zeta$, uniformly in $\varepsilon \in D(0, R)$ : there exist $A, B$ in $] 0,+\infty[$ such that

$$
|h(\zeta, \varepsilon)| \leq A e^{B|\zeta|} \quad \text { for all }(\zeta, \varepsilon) \in \mathfrak{C}_{m} \times D(0, R) .
$$

This last result, together with (3-26), shows that the series expansion $\widetilde{\psi}_{n}(\zeta, \underline{a}, \varepsilon)$ converges uniformly for $\zeta$ in every compact set of $\Omega_{m}(\rho), \underline{a} \in K$ and $\varepsilon \in D(0, R)$, and moreover,

$$
|\widetilde{\psi}(\zeta, \underline{a}, \varepsilon)| \leq \tilde{h}(|\zeta|,|\varepsilon|) \leq A e^{B|\zeta|} \quad \text { for all }(\zeta, \underline{a}, \varepsilon) \in \Omega_{m}(\rho) \times K \times D(0, R) .
$$

Putting $\varepsilon=1$, we deduce the same result for $\widetilde{\psi}_{+}(\zeta, \underline{a})$ : holomorphy in $\Omega_{m}(\rho) \times K$, exponential growth of order not greater than 1 at infinity in $\zeta$, uniformly in $\underline{a} \in K$. Since $\rho>0$ can be chosen arbitrarily small, we have shown that, except for the directions of argument $\alpha=\pi(\bmod 2 \pi)$, there is no singular point on the half line $\arg \zeta=\alpha$ and, since $\widetilde{\psi}_{+}(\zeta, \underline{a})$ has an exponential growth of order not greater than 1 at infinity in $\zeta$, uniformly in $\underline{a} \in K$, we deduce that $\psi_{+}(z, \underline{a})$ is Borel-resummable with respect to $z$, uniformly in $\underline{a}$ for $\underline{a}$ in any compact set of $\mathbb{C}^{m}$, for every direction of summation except those of argument $\pi(\bmod 2 \pi)$.

Thanks to (3-13), an analogous result can be obtained $\psi_{-}(z, \underline{a})$. This yields Proposition 3.16 .

Proposition 3.16 is enough to prove Theorem 2.1. Let us define $\phi_{0}(x, \underline{a}) \in$ $\mathbb{C}[\underline{a}] \llbracket x^{-1 / 2} \rrbracket$ by the formula

$$
x^{r(\underline{a})} e^{-S(x, \underline{a})} \phi_{0}(x, \underline{a})=\left.\frac{\sqrt{x}}{P_{m}(x, \underline{a})^{\frac{1}{4}}} e^{-z} \psi_{+}(z, \underline{a})\right|_{z=z(x, \underline{a}) .}
$$

Due to the definition of $\psi_{+}$, the left-hand side of this equality is a formal solution of equation $\left(\mathfrak{E}_{m}\right)$.

We know from Proposition 3.16 that $\psi_{+}(z, \underline{a})$ is Borel-resummable for the direction of argument 0 . For $\underline{a}$ in any given compact set $K$ of $\mathbb{C}^{m}$, this allows us to 
define the function

$$
\Phi_{0}(x, \underline{a})=\left.\frac{\sqrt{x}}{P_{m}(x, \underline{a})^{1 / 4}} e^{-z} \mathrm{~S}_{0} \psi_{+}(z, \underline{a})\right|_{z=z(x, \underline{a}),}
$$

which is an analytic solution of $\left(\mathfrak{E}_{m}\right)$ for $z$ in a sectorial neighbourhood of infinity of aperture $]-\frac{\pi}{2}, \frac{\pi}{2}$ [ and $\underline{a}$ in $K$. Note that the size of the sectorial neighbourhood may depend on $K$. By the inverse map $z \leftrightarrow x$ given by (3-5), this corresponds to an $x$-sectorial neighbourhood of infinity of aperture ] $-\frac{\pi}{m}, \frac{\pi}{m}$ [. From Proposition 3.16 again, $\Phi_{0}$ can be analytically extended by varying the direction of summation on ] $-\pi, \pi$ [. This shows that $\Phi_{0}$ is holomorphic in an $x$-sectorial neighbourhood of infinity $\Sigma_{0}^{\prime}$, of aperture $]-\frac{3 \pi}{m}, \frac{3 \pi}{m}$ [ and, by construction, $\Phi_{0}$ is asymptotic to $x^{r(\underline{a})} e^{-S(x, \underline{a})} \phi_{0}(x, \underline{a})$ at infinity in $\Sigma_{0}^{\prime}$, uniformly in $\underline{a} \in K$. The uniqueness of $\Phi_{0}$ follows from the Watson theorem [Malgrange 1995].

Also, since for any strict sub-sector $\Sigma$ of $\Sigma_{0}$ the set $\Sigma \backslash \Sigma \cap \Sigma_{0}^{\prime}$ is bounded, all we have to do now to prove part (1) of Theorem 2.1 is to show that $\Phi_{0}$ extends analytically in $x \in \Sigma_{0}$. This is a consequence of the Cauchy-Kovalevskaya theorem: take a point $x_{0}$ in $\Sigma_{0}^{\prime}$ and consider the datum $\left(\Phi_{0}\left(x_{0}, \underline{a}\right), \Phi_{0}^{\prime}\left(x_{0}, \underline{a}\right)\right)$. Then $\Phi_{0}$ is uniquely defined by this Cauchy datum, which is holomorphic in $\underline{a} \in K$. Since the linear differential equation $\left(\mathfrak{E}_{m}\right)$ is holomorphic in $(x, \underline{a}) \in \mathbb{C}^{\star} \times \mathbb{C}$, we conclude that $\Phi_{0}$ extends analytically to $\widetilde{\mathbb{C}}^{\star} \times K$, where $\widetilde{\mathbb{C}}^{\star}$ stands for the universal covering of $\mathbb{C}^{\star}$. We end by noticing that $K$ can be chosen arbitrarily. This also proves part (2) of Theorem 2.1.

Part (3) of Theorem 2.1 follows from the fact that the Borel resummation with respect to $z$ commutes with the derivative $\frac{d}{d z}$.

Note that besides proving Theorem 2.1, we have obtained the following interesting result.

Proposition 3.17. If $m$ is odd, the analytic function $\Phi_{0}$ of Theorem 2.1 is given by

$$
\Phi_{0}(x, \underline{a})=\left.\frac{\sqrt{x}}{P_{m}(x, \underline{a})^{\frac{1}{4}}} e^{-z} \mathrm{~S}_{\alpha} \psi_{+}(z, \underline{a})\right|_{z=z(x, \underline{a}),}
$$

for $x$ in a sectorial neighbourhood of infinity of aperture $]-\frac{\pi}{m}-\frac{2 \alpha}{m}, \frac{\pi}{m}-\frac{2 \alpha}{m}[$, uniformly in a for a in any compact set of $\mathbb{C}^{m}$, where the direction of Borel resummation $\alpha$ runs through $]-\pi,+\pi[$.

The arguments used to prove Proposition 3.16 can be extended to analyze the whole analytic structure of the minor $\widetilde{\psi}_{+}(\zeta, \underline{a})$ of $\psi_{+}(z, \underline{a})$. Since the techniques involved are the same as those used in [Loday-Richaud 1995] and [Gelfreich and Sauzin 2001], we just give the final result. (For the particular reader, this part is detailed in [Rasoamanana 2006].) 
Proposition 3.18. The minor $\widetilde{\psi}_{+}(\zeta, \underline{a}) \in \mathbb{C}[\underline{a}]\left\{\zeta^{1 / m}\right\}$ (respectively, $\widetilde{\psi}_{-}(\zeta, \underline{a}) \in$ $\left.\mathbb{C}[\underline{a}]\left\{\zeta^{1 / m}\right\}\right)$ of $\psi_{+}(z, \underline{a})$ (respectively, $\psi_{-}(z, \underline{a})$ ) can be extended analytically to $(\zeta, \underline{a}) \in \widetilde{\mathbb{C}} \backslash\{0,-2\} \times \mathbb{C}^{m}$ (respectively, $(\zeta, \underline{a}) \in \widetilde{\mathbb{C}} \backslash\{0,+2\} \times \mathbb{C}^{m}$ ), where $\widetilde{\mathbb{C}} \backslash\{0, \pm 2\}$ is the universal covering of $\mathbb{C} \backslash\{0, \pm 2\}$. Moreover, $\widetilde{\psi}_{ \pm}$has an exponential growth of order not greater than 1 at infinity in $\zeta$, uniformly in a for a in any given compact set of $\mathbb{C}^{m}$.

One can make things more precise concerning the resurgent structure, that is, the behavior of $\widetilde{\psi}_{+}$and $\widetilde{\psi}_{-}$near their singular points. To do that, we shall use the alien derivations. We would like to compute $\Delta_{\tau} \psi_{+}$, where $\Delta_{\tau}$ stands for the alien derivation at $\tau$. From Proposition 3.18 we know that the singular points of the minor of $\psi_{+}$lie above -2 and 0 . However, since $\psi_{+}$belongs to $\mathbb{C}[\underline{a}] \llbracket z^{-1 / m} \rrbracket$, the nonvanishing $\Delta_{\tau} \psi_{+}$can be indexed by the elements $\tau$ above -2 on the Riemann surface $\mathfrak{C}_{m}$ of $z^{1 / m}$. We now use one of the fundamental properties of the alien derivations: the pointed alien derivation $\dot{\Delta}_{\tau}=e^{-\tau z} \Delta_{\tau}$ commutes with $\frac{d}{d z}$ (Proposition 3.15). Using the facts that the resurgent symbol (Definition 3.9) $\Psi_{+}(z, \underline{a})=e^{-z} \psi_{+}(z, \underline{a})$ is solution of (3-4), and that $F$ is a constant of resurgence (Definition 3.14), we obtain

$$
-\frac{d^{2}}{d z^{2}}\left(\dot{\Delta}_{\tau} \Psi_{+}\right)+(1-F(z, \underline{a}))\left(\dot{\Delta}_{\tau} \Psi_{+}\right)=0
$$

This means that $\dot{\Delta}_{\tau} \Psi_{+}$satisfies the same equation (3-4). Since $\left(\Psi_{+}, \Psi_{-}\right)$is a fundamental system of formal solutions for (3-4), we can conclude that $\dot{\Delta}_{\tau} \Psi_{+}$has to be proportional to the resurgent symbol $\Psi_{-}$by an argument of singular support (Definition 3.9): the singular support of $\Psi_{+}$(respectively, $\Psi_{-}$) reduces to $\{+1\}$ (respectively, $\{-1\}$ ), whereas, by definition, the resurgent symbol

$$
\dot{\Delta}_{\tau} \Psi_{+}=e^{+z} \times(\text { a formal resurgent function })
$$

has $\{-1\}$ for its singular support. We deduce that there is $\delta_{\tau}(\underline{a})$ such that

$$
\dot{\Delta}_{\tau} \Psi_{+}(z, \underline{a})=\delta_{\tau}(\underline{a}) \Psi_{-}(z, \underline{a}),
$$

that is, $\Delta_{\tau} \psi_{+}(z, \underline{a})=\delta_{\tau}(\underline{a}) \psi_{-}(z, \underline{a})$. Similarly, one obtains the existence of $\delta_{\tau}(\underline{a})$ such that $\Delta_{\tau} \psi_{-}(z, \underline{a})=\delta_{\tau}(\underline{a}) \psi_{+}(z, \underline{a})$, where $\tau$ is above +2 on the Riemann surface $\mathfrak{C}_{m}$.

The coefficients $\delta_{\tau}(\underline{a})$ are entire functions of $\underline{a}$ : this stems directly from the regularity in $\underline{a}$ of the formal series $\psi_{+}, \psi_{-}$, and from the fact that the location of the singular points of the minors does not depend on $\underline{a}$, so that the Stokes automorphism (in any direction) commutes with the analytic continuation in $\underline{a}$. However, this is a consequence of Theorem 3.27 which will be discussed in a moment.

To summarize: 
Theorem 3.19. For $m$ odd, there exists a unique formal power series $\psi_{+}(z, \underline{a}) \in$ $\mathbb{C}[\underline{a}] \llbracket z^{-1 / m} \rrbracket$ (respectively, $\left.\psi_{-}(z, \underline{a}) \in \mathbb{C}[\underline{a}] \llbracket z^{-1 / m} \rrbracket\right)$ whose residual coefficient is 1 , such that $e^{-z} \psi_{+}(z, \underline{a})$ (respectively, $e^{+z} \psi_{-}(z, \underline{a})$ ) is a solution of (3-4) and, moreover,

$$
\psi_{-}(z, \underline{a})=\psi_{+}\left(\omega^{m / 2} z, \omega \cdot \underline{a}\right) .
$$

These formal power series $\psi_{ \pm}$are resurgent in $z$ with holomorphic dependence in $\underline{a}$, and Borel-resummable in $z$, uniformly with respect to $\underline{a}$ in any compact set (except, of course, for the singular directions which are described by the resurgence structure). Their resurgent structure is given by

$$
\begin{aligned}
\Delta_{2 e^{k i \pi}} \psi_{-}(z, \underline{a}) & =S_{k}(\underline{a}) \psi_{+}(z, \underline{a}) & & \text { for } k \in 2 \mathbb{Z}, \\
\Delta_{2 e^{k i \pi}} \psi_{+}(z, \underline{a}) & =S_{k}(\underline{a}) \psi_{-}(z, \underline{a}) & & \text { for } k-1 \in 2 \mathbb{Z}, \\
\Delta_{\tau} \psi_{ \pm} & =0 & & \text { otherwise, }
\end{aligned}
$$

where $\Delta_{\tau}$ is the alien derivation at $\tau$. The coefficients $S_{k}(\underline{a})$ are entire functions in $\underline{a}$ and, for all $k \in \mathbb{Z}, S_{k}=S_{k(\bmod 2 m)}$.

Definition 3.20. The coefficients $S_{k}(\underline{a})$, for $k \in \mathbb{Z}$, are called the Stokes multipliers.

Case 2: $m$ even. The fundamental difference with the case where $m$ is odd is the existence of the term $b_{0}(\underline{a}) \ln (x)$ in the asymptotic expansion of $z(x, \underline{a})$ (defined by (3-2)) at infinity in $x$. This is why it is worth considering the following new Green-Liouville transformation

$$
\begin{aligned}
\tilde{z}=\tilde{z}(x, \underline{a})=\int \frac{\sqrt{P_{m}(t, \underline{a})}-b_{0}(\underline{a})}{t} d t, \\
\Psi(\tilde{z}, \underline{a})=\frac{\sqrt{\sqrt{P_{m}(x, \underline{a})}-b_{0}(\underline{a})}}{\sqrt{x}} \Phi(x, \underline{a})
\end{aligned}
$$

so that the Laurent-Puiseux series expansion of $x \mapsto \tilde{z}(x, \underline{a})$ coincides with the map $x \mapsto S(x, \underline{a})$ defined in Theorem 2.1 modulo an analytic function vanishing at infinity. The quasi-homogeneity properties (3-3) and (3-6) are still valid for the maps $(x, \underline{a}) \mapsto \tilde{z}(x, \underline{a})$ and $(\tilde{z}, \underline{a}) \mapsto x(\tilde{z}, \underline{a})$ respectively.

Equation $\left(\mathfrak{E}_{m}\right)$ is converted into the prepared equation

$$
-\frac{d^{2}}{d \tilde{z}^{2}} \Psi+\left(1+\frac{4 b_{0}(\underline{a})}{m \tilde{z}}-H(\tilde{z}, \underline{a})\right) \Psi=0
$$


with

(3-34)

$$
\begin{aligned}
H(\tilde{z}, a)=1 & +\frac{4 b_{0}(\underline{a})}{m \tilde{z}}-\frac{P_{m}(x, \underline{a})}{\left(\sqrt{P_{m}(x, \underline{a})}-b_{0}(\underline{a})\right)^{2}} \\
- & \left(\frac { x ^ { 2 } } { 4 } \left(\frac{P_{m}^{\prime \prime}(x, \underline{a})}{\sqrt{P_{m}(x, \underline{a})}\left(\sqrt{P_{m}(x, \underline{a})}-b_{0}(\underline{a})\right)^{3}}\right.\right. \\
& \left.-\frac{1}{4} \frac{\left(P_{m}^{\prime}(x, \underline{a})\right)^{2}\left(5 \sqrt{P_{m}(x, \underline{a})}-2 b_{0}(\underline{a})\right)}{\left(P_{m}(x, \underline{a})-b_{0}(\underline{a}) \sqrt{\left.P_{m}(x, \underline{a})\right)^{3}\left(\sqrt{P_{m}(x, \underline{a})}-b_{0}(\underline{a})\right)}\right.}\right) \\
& \left.\quad+\frac{x P_{m}^{\prime}(x, \underline{a})+P_{m}(x, \underline{a})-b_{0}(\underline{a}) \sqrt{P_{m}(x, \underline{a})}}{4 \sqrt{P_{m}(x, \underline{a})}\left(\sqrt{P_{m}(x, \underline{a})}-b_{0}(\underline{a})\right)^{3}}\right)\left.\right|_{\tilde{z}=\tilde{z}(x, \underline{a})}
\end{aligned}
$$

and

$$
H(\tilde{z}, \underline{a})=\frac{m^{2}-4}{4 m^{2} \tilde{z}^{2}}\left(1+O\left(\tilde{z}^{-2 / m}\right)\right) \in \frac{1}{\tilde{z}^{2}} \mathbb{C}[\underline{a}]\left\{\tilde{z}^{-2 / m}\right\} .
$$

Furthermore, $H$ satisfies the quasi-homogeneity property (3-12).

One easily proves the existence of a unique formal solution

$$
\Psi_{+}(\tilde{z}, \underline{a})=e^{-\tilde{z}} \psi_{+}(\tilde{z}, \underline{a})
$$

of (3-33), satisfying

$$
\psi_{+}(\tilde{z}, \underline{a})=\tilde{z}^{-2 b_{0}(\underline{a}) / m} \mu_{+}(\tilde{z}, \underline{a})
$$

where $\mu_{+} \in \mathbb{C}[\underline{a}] \llbracket \tilde{z}^{-2 / m} \rrbracket$ with residual coefficient 1 . By quasi-homogeneity, one deduces the existence of another formal solution

$$
\Psi_{-}(\tilde{z}, \underline{a})=e^{+\tilde{z}} \psi_{-}(\tilde{z}, \underline{a})=e^{+\tilde{z}} \tilde{z}^{+2 b_{0}(\underline{a}) / m} \mu_{-}(\tilde{z}, \underline{a})
$$

of (3-33) such that

$$
\psi_{-}(\tilde{z}, \underline{a})=\psi_{+}\left(\omega^{m / 2} \tilde{z}, \omega \cdot \underline{a}\right) .
$$

From now on the analysis is exactly the same as in the case where $m$ is odd, and yields the following results.

Theorem 3.21. For $m$ even, there exists a unique formal series

$$
\psi_{+}(\tilde{z}, \underline{a})=\tilde{z}^{-2 b_{0}(\underline{a}) / m} \mu_{+}(\tilde{z}, \underline{a})
$$

where $\mu_{+} \in \mathbb{C}[\underline{a}] \llbracket \tilde{z}^{-2 / m} \rrbracket$ with residual coefficient 1 (respectively,

$$
\psi_{-}(\tilde{z}, \underline{a})=\tilde{z}^{+2 b_{0}(\underline{a}) / m} \mu_{-}(\tilde{z}, \underline{a})
$$


where $\mu_{-} \in \mathbb{C}[\underline{a}] \llbracket \tilde{z}^{-2 / m} \rrbracket$ with residual coefficient 1$)$, such that $e^{-\tilde{z}} \psi_{+}(\tilde{z}, \underline{a})$ (respectively, $\left.e^{+\tilde{z}} \psi_{-}(\tilde{z}, \underline{a})\right)$ is solution of (3-33). Moreover,

$$
\psi_{-}(\tilde{z}, \underline{a})=\psi_{+}\left(\omega^{m / 2} \tilde{z}, \omega \cdot \underline{a}\right) .
$$

The formal power series $\psi_{ \pm}$are resurgent in $\tilde{z}$ with holomorphic dependence in $\underline{\text { a }}$, and Borel-resummable in $\tilde{z}$, uniformly with respect to $\underline{a}$ in any compact set. There exists a set of entire functions $S_{k}(\underline{a})$, the Stokes multipliers, such that

$$
\begin{aligned}
\Delta_{2 e^{k i \pi}} \psi_{-}(\tilde{z}, \underline{a}) & =S_{k}(\underline{a}) \psi_{+}(\tilde{z}, \underline{a}) & & \text { for } k \in 2 \mathbb{Z}, \\
\Delta_{2 e^{k i \pi}} \psi_{+}(\tilde{z}, \underline{a}) & =S_{k}(\underline{a}) \psi_{-}(\tilde{z}, \underline{a}) & & \text { for } k-1 \in 2 \mathbb{Z}, \\
\Delta_{\tau} \psi_{ \pm} & =0 & & \text { otherwise, }
\end{aligned}
$$

where $\Delta_{\tau}$ is the alien derivation at $\tau$.

In this theorem, due to the fact that the formal solutions $\mu_{+}$and $\mu_{-}$belong to $\mathbb{C}[\underline{a}] \llbracket z^{-2 / m} \rrbracket$, the alien derivations need only to be indexed by elements on the Riemann surface of $z^{2 / m}$. Thus, a priori, only $m$ Stokes multipliers govern the resurgence structure. Nevertheless, it is better to describe the resurgence structure in terms of $\psi_{+}$and $\psi_{-}$, which have to be thought of as formal functions on the universal covering of $\mathbb{C}^{\star}$.

Returning to Theorem 2.1, we finally get the desired result.

Proposition 3.22. If $m$ is even, the analytic function $\Phi_{0}$ of Theorem 2.1 is given by

$$
\Phi_{0}(x, \underline{a})=\left.\frac{\sqrt{x}}{\left(\sqrt{P_{m}(x, \underline{a})}-b_{0}(\underline{a})\right)^{\frac{1}{2}}} e^{-\tilde{z}} \mathrm{~S}_{\alpha} \psi_{+}(\tilde{z}, \underline{a})\right|_{\tilde{z}=\tilde{z}(x, \underline{a}),}
$$

for $x$ in a sectorial neighbourhood of infinity of aperture $]-\frac{\pi}{m}-\frac{2 \alpha}{m}, \frac{\pi}{m}-\frac{2 \alpha}{m}[$, uniformly in a for a in any compact set of $\mathbb{C}^{m}$, where the direction of Borel resummation $\alpha$ runs through $]-\pi,+\pi[$.

Some properties of the Stokes multipliers. The quasi-homogeneity induces some interesting properties of the Stokes multipliers. To simplify, we introduce the following notation.

\section{Notation 3.23.}

$$
\omega=e^{2 i \pi / m} .
$$

We recall the following easy result in resurgence theory; see [Écalle 1981].

Lemma 3.24. Let $\psi_{1}(y)$ be a formal resurgent function and let $v$ be a nonzero complex number. Setting $y=v t$ and $\psi_{2}(t)=\psi_{1}(y)$, we have

$$
\Delta_{v \tau}^{t} \psi_{2}=\Delta_{\tau}^{y} \psi_{1}
$$


where $\Delta_{\tau}^{x}$ denotes the alien derivation at $\tau$ with respect to the variable $x$.

Proposition 3.25. With the notations of Theorems 3.19 and 3.21, we have, for all $k \in \mathbb{Z}$,

$$
S_{k}(\underline{a})=S_{0}\left(\omega^{k} \cdot \underline{a}\right) .
$$

Proof. In Theorems 3.19 and 3.21 we introduce $t=z$ for $m$ odd, and $t=\tilde{z}$ for $m$ even. From (3-31) and (3-36), we get

$$
\begin{aligned}
\psi_{+}\left(e^{+i \pi} t, \omega \cdot \underline{a}\right) & =\psi_{-}(t, \underline{a}), \\
\psi_{+}(t, \underline{a}) & =\psi_{-}\left(e^{-i \pi} t, \omega^{-1} \cdot \underline{a}\right) .
\end{aligned}
$$

Using Lemma 3.24 with $y=e^{i \pi} t$, we deduce

$$
\Delta_{2 e^{i 0}}^{y} \psi_{-}(y, \omega \cdot \underline{a})=\Delta_{2 e^{i \pi}}^{t} \psi_{+}(t, \underline{a}) .
$$

Now, by the definition of $S_{0}$ and $S_{1}$ (Theorems 3.19 and 3.21), we have

$$
\begin{aligned}
\Delta_{2 e^{i 0}}^{y} \psi_{-}(y, \omega . \underline{a}) & =S_{0}(\omega \cdot \underline{a}) \psi_{+}(y, \omega \cdot \underline{a}), \\
\Delta_{2 e^{i \pi}}^{z} \psi_{+}(t, \underline{a}) & =S_{1}(\underline{a}) \psi_{-}(t, \underline{a}) .
\end{aligned}
$$

Finally, we obtain

$$
S_{1}(\underline{a})=S_{0}(\omega \cdot \underline{a}) .
$$

We complete the proof with an easy induction argument.

Since $\omega^{m}=e^{2 i \pi}$, we get the following corollary.

Corollary 3.26. For all $k \in \mathbb{Z}$,

$$
S_{k}=S_{k(\bmod m)} .
$$

Stokes-Sibuya coefficients and Stokes multipliers. In order to describe the connection formulas, we now have two sets of Stokes coefficients at our disposal. One is made up of the Stokes-Sibuya coefficients $C_{k}(\underline{a})$, the other is made up of the Stokes multipliers $S_{k}(\underline{a})$. The following proposition clarifies the relations between these two fundamental data.

Theorem 3.27. We consider the Stokes-Sibuya coefficients $C_{k}$ given by Theorem 2.7 and the Stokes multipliers described by Theorems 3.19 and 3.21. Then

$$
S_{k}(\underline{a})=\omega^{r\left(\omega^{k} \cdot \underline{a}\right)} C_{k}(\underline{a})
$$

for all $k \in \mathbb{Z}$, where $\omega$ is given by (3-38).

Here, $r(\underline{a})$ is as defined in Theorem 2.1. In particular, when $m$ is odd, then $r(\underline{a})=\frac{1}{2}-\frac{m}{4}$ does not depend on $\underline{a}$, so (3-39) becomes

$$
S_{k}(\underline{a})=\omega^{r} C_{k}(\underline{a}) .
$$


Proof. To simplify, we give the proof for odd $m$ only, so that $r=\frac{1}{2}-\frac{m}{4}$.

By Proposition 3.25, and (2-7) in Theorem 2.7, it is sufficient to show (3-39) for $k=0$. By Proposition 3.17, $\Phi_{0}$ of Theorem 2.1 can be defined by

$$
\Phi_{0}(x, \underline{a})=\left.\frac{\sqrt{x}}{P_{m}(x, \underline{a})^{1 / 4}} e^{-z} \mathrm{~S}_{0} \psi_{+}(z, \underline{a})\right|_{z=z(x, \underline{a}),}
$$

for $z$ in a sectorial neighbourhood of infinity of aperture $]-\frac{\pi}{2}, \frac{\pi}{2}[$, which corresponds to $x$ in a sectorial neighbourhood of infinity of aperture $]-\frac{\pi}{m}, \frac{\pi}{m}[$. Now, by Theorem 2.7, $\Phi_{1}$ is defined by

$$
\Phi_{1}(x, \underline{a})=\Phi_{0}(\omega x, \omega . \underline{a}) .
$$

Using (3-40), we get the representation

$$
\Phi_{1}(x, \underline{a})=\left.\frac{\sqrt{x \omega}}{P_{m}(x \omega, \omega \cdot \underline{a})^{1 / 4}} e^{-z} \mathrm{~S}_{0} \psi_{+}(z, \omega \cdot \underline{a})\right|_{z=z(\omega x, \omega \cdot \underline{a}),}
$$

for $\Phi_{1}$, where $\omega x$ lies in a sectorial neighbourhood of infinity of aperture $]-\frac{\pi}{m}, \frac{\pi}{m}[$, that is, $x$ lies in a sectorial neighbourhood of infinity of aperture $]-\frac{3 \pi}{m},-\frac{\pi}{m}[$, so that $z$ belongs to a sectorial neighbourhood of infinity of aperture $]-\frac{3 \pi}{2},-\frac{\pi}{2}[$. By quasi-homogeneity considerations, we have seen that $z(\omega x, \omega \cdot \underline{a})=e^{i \pi} z(x, \underline{a})$, (see (3-3)), so that, by (3-31) in Theorem 3.19,

$$
\psi_{+}(z(\omega x, \omega \cdot \underline{a}), \omega \cdot \underline{a})=\psi_{-}(z, \underline{a}) .
$$

Also,

$$
P_{m}(\omega x, \omega \cdot \underline{a})=\omega^{m} P_{m}(x, \underline{a}) .
$$

This means that (3-41) can be written as

$$
\Phi_{1}(x, \underline{a})=\left.\omega^{r} \frac{\sqrt{x}}{P_{m}(x, \underline{a})^{1 / 4}} e^{z} \mathrm{~S}_{\pi} \psi_{-}(z, \underline{a})\right|_{z=z(x, \underline{a})}
$$

for $x$ in a sectorial neighbourhood of infinity of aperture $]-\frac{3 \pi}{m},-\frac{\pi}{m}[$ and $z$ in a sectorial neighbourhood of infinity of aperture $]-\frac{3 \pi}{2},-\frac{\pi}{2}\left[\right.$. As for $\Phi_{1}$, we have the representation

$$
\Phi_{-1}(x, \underline{a})=\left.\omega^{-r} \frac{\sqrt{x}}{P_{m}(x, \underline{a})^{1 / 4}} e^{z} \mathrm{~S}_{-\pi} \psi_{-}(z, \underline{a})\right|_{z=z(x, \underline{a})}
$$

for $\Phi_{-1}$, for $x$ in a sectorial neighbourhood of infinity of aperture $] \frac{\pi}{m}, \frac{3 \pi}{m}[$ and $z$ in a sectorial neighbourhood of infinity of aperture $] \frac{\pi}{2}, \frac{3 \pi}{2}[$.

To compare $\Phi_{-1}, \Phi_{0}$, and $\Phi_{1}$, we rotate the directions of resummation so as to sum along the direction 0 . Since $\Delta_{2 e^{i 0}} \psi_{-}(z, \underline{a})=S_{0}(\underline{a}) \psi_{+}(z, \underline{a})$ (see Theorem 3.19), $\psi_{-}$is not Borel-resummable in the direction 0 if $S_{0}(\underline{a}) \neq 0$, but only right 
or left Borel-resummable. In other words, we have to take into account a Stokes phenomenon. Since $\underline{\Delta}_{0} \psi_{-}(z, \underline{a})$ (Definition 3.11$)$ reduces to $\dot{\Delta}_{2 e^{i 0}} \psi_{-}(z, \underline{a})$, one gets

$$
\mathfrak{S}_{0} \psi_{-}(z, \underline{a})=\psi_{-}(z, \underline{a})+\dot{\Delta}_{2 e^{i 0}} \psi_{-}(z, \underline{a}),
$$

where $\mathfrak{S}_{0}$ is the Stokes automorphism in the direction 0 (Definition 3.10). Therefore,

$$
\mathrm{S}_{0-} \psi_{-}(z, \underline{a})=\mathrm{S}_{0+}\left[\psi_{-}(z, \underline{a})+e^{-2 z} S_{0}(\underline{a}) \psi_{+}(z, \underline{a})\right],
$$

where $\mathrm{S}_{0+}$ (respectively, $\mathrm{S}_{0_{-}}$) is the right (respectively, left) Borel-resummation in the direction 0 .

We obtain

$$
\begin{aligned}
\Phi_{0}(x, \underline{a}) & =\left.\frac{\sqrt{x}}{P_{m}(x, \underline{a})^{1 / 4}} e^{-z} \mathrm{~S}_{0} \psi_{+}(z, \underline{a})\right|_{z=z(x, \underline{a}),} \\
\Phi_{1}(x, \underline{a}) & =\left.\omega^{r} \frac{\sqrt{x}}{P_{m}(x, \underline{a})^{1 / 4}} e^{+z} \mathrm{~S}_{0+} \psi_{-}(z, \underline{a})\right|_{z=z(x, \underline{a}),} \\
\Phi_{-1}(x, \underline{a}) & =\left.\omega^{-r} \frac{\sqrt{x}}{P_{m}(x, \underline{a})^{1 / 4}} \mathrm{~S}_{0+}\left(e^{z} \psi_{-}(z, \underline{a})+e^{-z} S_{0}(\underline{a}) \psi_{+}(z, \underline{a})\right)\right|_{z=z(x, a) .}
\end{aligned}
$$

By Theorem 2.7, we have the connection formula $\Phi_{-1}=C_{0}(\underline{a}) \Phi_{0}+\widetilde{C}_{0}(\underline{a}) \Phi_{1}$; in this equality, replacing $\Phi_{-1}, \Phi_{0}$ and $\Phi_{1}$ by the right-hand sides of (3-42) and equating the coefficients of $e^{-z} \mathrm{~S}_{0} \psi_{+}$and $e^{+z} \mathrm{~S}_{0^{+}} \psi_{-}$, we finally get

$$
\begin{aligned}
& S_{0}(\underline{a})=\omega^{r} C_{0}(\underline{a}), \\
& \widetilde{C}_{0}(\underline{a})=\omega^{-2 r}=\omega^{m-2+2 r},
\end{aligned}
$$

which completes the proof.

\section{Solutions of $\left(\mathfrak{E}_{m}\right)$ in the neighbourhood of the origin: Fuchs theory}

In order to get more information about the Stokes-Sibuya coefficients $C_{k}$ (or about the Stokes multipliers $S_{k}$, since this is equivalent by Theorem 3.27), we have to pick up the necessary information coming from the other singular point of $\left(\mathfrak{E}_{m}\right)$, that is, the origin.

Since the origin is a regular singular point of $\left(\mathfrak{E}_{m}\right)$, the classical Fuchs theory allows to describe "canonical" systems of solutions of $\left(\mathfrak{E}_{m}\right)$ near the origin (see, for example, [Reinhard 1982; Wasow 1965]). The characteristic equation is

$$
s(s-1)-a_{m}=0,
$$

so that $\frac{1 \pm p}{2}$ are the characteristic values, with $p=\left(1+4 a_{m}\right)^{1 / 2}$. 
Notation 4.1. In what follows, $p=\left(1+4 a_{m}\right)^{1 / 2}$ and $s(p)=\frac{1+p}{2}$. We denote $\underline{a}^{\prime}:=\left(a_{1}, \ldots, a_{m-1}\right)$ and, for all $\tau \in \mathbb{C}$,

$$
\tau \cdot \underline{a}^{\prime}:=\left(\tau a_{1}, \ldots, \tau^{m-1} a_{m-1}\right) .
$$

As is well-known, we have to distinguish between the case $p \notin \mathbb{Z}$, the case $p \in \mathbb{Z}^{\star}$ and the case $p=0$. Since we have the freedom for choosing the determination of the square root $\left(1+4 a_{m}\right)^{1 / 2}$, we can avoid the case where $p \in-\mathbb{N}^{\star}$ in the following theorem.

Theorem 4.2. There exist two unique linearly independent solutions, $f_{1}$ and $f_{2}$, of $\left(\mathfrak{E}_{m}\right)$ such that

$$
\begin{aligned}
& f_{1}\left(x, \underline{a}^{\prime}, p\right)=x^{s(p)} g_{1}\left(x, \underline{a}^{\prime}, p\right)=x^{s(p)}\left(1+\sum_{k=1}^{\infty} A_{k}\left(\underline{a}^{\prime}, p\right) x^{k}\right) \\
& f_{2}\left(x, \underline{a}^{\prime}, p\right)=\lambda\left(\underline{a}^{\prime}, p\right) f_{1}\left(x, \underline{a}^{\prime}, p\right) \ln (x)+x^{s(-p)} g_{2}\left(x, \underline{a}^{\prime}, p\right)
\end{aligned}
$$

where $g_{1}$ and $g_{2}$ are entire functions in $x$ and $\underline{a}^{\prime}$, while $\lambda$ is entire in $\underline{a}^{\prime}$. Moreover, $g_{1}$ is meromorphic in $p$ with at most simple poles when $-p \in \mathbb{N}^{\star}$. Precisely, $A_{k}\left(\underline{a}^{\prime}, p\right) \prod_{l=1}^{k}(p+l) \in \mathbb{C}\left[\underline{a}^{\prime}, p\right]$ for all $k \in \mathbb{N}^{\star}$.

(1) When $p \notin \mathbb{Z}$, then $\lambda\left(\underline{a}^{\prime}, p\right)=0$ and $g_{2}\left(x, \underline{a}^{\prime}, p\right)=g_{1}\left(x, \underline{a}^{\prime},-p\right)$.

(2) When $p \in \mathbb{N}^{\star}$, then

$$
g_{2}\left(x, \underline{a}^{\prime}, p\right)=\left(1+\sum_{k=1}^{\infty} B_{k}\left(\underline{a}^{\prime}, p\right) x^{k}\right) \text { with } B_{p}=0 .
$$

Moreover, $B_{k}\left(\underline{a}^{\prime}, p\right) \in \mathbb{C}\left[\underline{a}^{\prime}\right]$ for all $k \in \mathbb{N}^{\star}$ and all $\lambda\left(\underline{a}^{\prime}, p\right)$, and

$$
\lambda\left(\omega . \underline{a}^{\prime}, p\right)=\omega^{-p} \lambda\left(\underline{a}^{\prime}, p\right) .
$$

(3) When $p=0$, then $\lambda\left(\underline{a}^{\prime}, p\right)=1$ and

$$
g_{2}\left(x, \underline{a}^{\prime}, p\right)=\sum_{k=1}^{\infty} B_{k}\left(\underline{a}^{\prime}, p\right) x^{k}
$$

with $B_{k}\left(\underline{a}^{\prime}, p\right) \in \mathbb{C}\left[\underline{a}^{\prime}\right]$ for all $k \in \mathbb{N}^{\star}$.

Remark 4.3. When $-p \in \mathbb{N}^{\star}$, just change $p$ into $-p$ in Theorem 4.2, which corresponds to choosing the other root for $\left(1+4 a_{m}\right)^{1 / 2}$ or, equivalently, to $a_{m}$ making a loop around $-\frac{1}{4}$.

Remark 4.4. In the special case when $\underline{a}^{\prime}=0$, the function $g_{1}$ is meromorphic in $p$ with at most simple poles when $-p \in m \mathbb{N}^{\star}$. 
The existence and unicity of $f_{1}$ and $f_{2}$ follow from the Fuchs theory, and the chosen normalization for $g_{1}$ and $g_{2}$. The properties of the coefficients $A_{k}, B_{k}$ and $\lambda$ can be proved by induction, and this induces the analytic properties of $g_{1}, g_{2}$. The quasi-homogeneity property of $\lambda$ is a consequence of the quasi-homogeneity of equation $\left(\mathfrak{E}_{m}\right)$.

The following result can be shown also by induction; see [Rasoamanana 2006].

Proposition 4.5. We consider $p \in \mathbb{N}^{\star}$ and let $p=k m+r$, for $0 \leq r \leq m-1$, its Euclidean division by $m$. We introduce

$$
\epsilon(r)= \begin{cases}1 & \text { if } r \neq 0 \\ 0 & \text { if } r=0\end{cases}
$$

Then

$$
\lambda\left(\underline{a}^{\prime}, p\right)=\frac{1}{p} \sum_{l=k+\epsilon(r)}^{p} \sum_{\substack{i_{1}+\ldots+i_{l}=p \\ 1 \leq i_{j} \leq m}} \frac{a_{m-i_{1}} \ldots a_{m-i_{l}}}{i_{1}\left(i_{1}-p\right) \ldots\left(i_{1}+\cdots+i_{l-1}\right)\left(i_{1}+\cdots+i_{l-1}-p\right)}
$$

with the convention $a_{0}=1$.

Remark 4.6. Let $p \in \mathbb{N}^{\star}$.

If $\underline{a}^{\prime}=0$, then

$$
\left.\lambda(0, p)\right|_{p \neq 0(\bmod m)}=0
$$

while, for $k \in \mathbb{N}^{\star}$,

$$
\left.\lambda(0, p)\right|_{p=k m}=\frac{(-1)^{k+1}}{m^{2 k-1} k \Gamma(k)^{2}} .
$$

When $m=2$, then

$$
\begin{aligned}
& \left.\lambda\left(\underline{a}^{\prime}, p\right)\right|_{p=0(\bmod 2)}=-\frac{1}{p \Gamma(p)^{2}} \prod_{k=1}^{p / 2}\left(a_{1}+2 k-1\right)\left(a_{1}-2 k+1\right) \\
& \left.\lambda\left(\underline{a}^{\prime}, p\right)\right|_{p=1(\bmod 2)}=\frac{a_{1}}{p \Gamma(p)^{2}} \prod_{k=1}^{(p-1) / 2}\left(a_{1}+2 k\right)\left(a_{1}-2 k\right)
\end{aligned}
$$

From the uniqueness of $f_{1}$ and $f_{2}$ in Theorem 4.2, and from the quasi-homogeneity of equation $\left(\mathfrak{E}_{m}\right)$, we easily obtain the following corollary.

Corollary 4.7. Consider the fundamental system of solutions $\left(f_{1}, f_{2}\right)$ of Theorem 4.2. Then

$$
\left(\begin{array}{l}
f_{1} \\
f_{2}
\end{array}\right)\left(\omega x, \omega \cdot \underline{a}^{\prime}, p\right)=\mathfrak{N}\left(\underline{a}^{\prime}, p\right)\left(\begin{array}{l}
f_{1} \\
f_{2}
\end{array}\right)\left(x, \underline{a}^{\prime}, p\right)
$$


with

$$
\mathfrak{N}\left(\underline{a}^{\prime}, p\right)=\left(\begin{array}{cc}
\omega^{s(p)} & 0 \\
\frac{2 i \pi}{m} \lambda\left(\underline{a}^{\prime}, p\right) \omega^{s(-p)} & \omega^{s(-p)}
\end{array}\right)
$$

Moreover

$$
\left(\begin{array}{l}
f_{1} \\
f_{2}
\end{array}\right)\left(\omega^{m} x, \underline{a}^{\prime}, p\right)=\mathfrak{M}\left(\underline{a}^{\prime}, p\right)\left(\begin{array}{c}
f_{1} \\
f_{2}
\end{array}\right)(x, a)
$$

where

$$
\mathfrak{M}\left(\underline{a}^{\prime}, p\right)=\left(\begin{array}{cc}
e^{2 i \pi s(p)} & 0 \\
2 i \pi \lambda\left(\underline{a}^{\prime}, p\right) e^{2 i \pi s(-p)} & e^{2 i \pi s(-p)}
\end{array}\right)
$$

is the monodromy matrix at the origin.

\section{The $0 \infty$ connection matrices}

In Section 2, we described a set of fundamental systems of solutions $\left(\Phi_{k-1}, \Phi_{k}\right)$ of $\left(\mathfrak{E}_{m}\right)$, where $k \in \mathbb{Z}$. In Section 4 , we have obtained another fundamental system of solutions $\left(f_{1}, f_{2}\right)$. To compare these fundamental systems, we introduce, for all $k \in \mathbb{Z}$,

$$
\left(\begin{array}{c}
\Phi_{k-1} \\
\Phi_{k}
\end{array}\right)(x, \underline{a})=M_{k}\left(\underline{a}^{\prime}, p\right)\left(\begin{array}{c}
f_{1} \\
f_{2}
\end{array}\right)\left(x, \underline{a}^{\prime}, p\right)
$$

where the matrices $M_{k}\left(\underline{a}^{\prime}, p\right)$ are invertible.

Definition 5.1. The matrices $M_{k}\left(\underline{a}^{\prime}, p\right)$ are called the $0 \infty$-connection matrices.

We now give some properties of the matrices $M_{k}$. These properties depend essentially on $p$, as does the fundamental system $\left(f_{1}, f_{2}\right)$.

We start with an obvious result.

Proposition 5.2. For every $k \in \mathbb{Z}$,

$$
M_{k+1}\left(\underline{a}^{\prime}, p\right)=M_{k}\left(\omega \cdot \underline{a}^{\prime}, p\right) \mathfrak{N}\left(\underline{a}^{\prime}, p\right)
$$

where the invertible matrix $\mathfrak{N}\left(\underline{a}^{\prime}, p\right)$ is given by (4-2).

Proof. By Theorem 2.7, we write

$$
\left(\begin{array}{c}
\Phi_{k} \\
\Phi_{k+1}
\end{array}\right)(x, \underline{a})=\left(\begin{array}{c}
\Phi_{k-1} \\
\Phi_{k}
\end{array}\right)(\omega x, \omega \cdot \underline{a})=M_{k}\left(\omega \cdot \underline{a}^{\prime}, p\right)\left(\begin{array}{c}
f_{1} \\
f_{2}
\end{array}\right)\left(\omega x, \omega \cdot \underline{a}^{\prime}, p\right) .
$$

Since, by the definition of $\mathfrak{N}$,

$$
\left(\begin{array}{l}
f_{1} \\
f_{2}
\end{array}\right)\left(\omega x, \omega \cdot \underline{a}^{\prime}, p\right)=\mathfrak{N}\left(\underline{a}^{\prime}, p\right)\left(\begin{array}{l}
f_{1} \\
f_{2}
\end{array}\right)\left(x, \underline{a}^{\prime}, p\right),
$$

we can conclude because $\left(f_{1}, f_{2}\right)$ is a fundamental system. 
Theorem 5.3. (a) For every $k \in \mathbb{Z}$,

$$
\operatorname{det} M_{k}\left(\underline{a}^{\prime}, p\right)= \begin{cases}2(-1)^{k} \omega^{(k-1)(1-m / 2)+r\left(\omega^{k} \cdot \underline{a}\right) / p} & \text { for } p \neq 0 \\ 2(-1)^{k-1} \omega^{(k-1)(1-m / 2)+r\left(\omega^{k} \cdot \underline{a}\right)} & \text { for } p=0 .\end{cases}
$$

(b) For every $k \in \mathbb{Z}$, the matrix $M_{k}\left(\underline{a}^{\prime}, p\right)$ is entire in $\underline{a}^{\prime}$. More precisely,

(5-4) $\quad M_{k}\left(\underline{a}^{\prime}, p\right)=\left(\begin{array}{cc}L_{k}\left(\underline{a}^{\prime}, p\right) & \widetilde{L}_{k}\left(\underline{a}^{\prime}, p\right) \\ \frac{2 i \pi}{m} \lambda\left(\underline{a}^{\prime}, p\right) \omega^{s(-p)} \widetilde{L}_{k}\left(\omega \cdot \underline{a}^{\prime}, p\right) & \omega^{s(-p)} \widetilde{L}_{k}\left(\omega \cdot \underline{a}^{\prime}, p\right) \\ +\omega^{s(p)} L_{k}\left(\omega \cdot \underline{a}^{\prime}, p\right) & \end{array}\right)$

where $L_{k}\left(\underline{a}^{\prime}, p\right)$ and $\widetilde{L}_{k}\left(\underline{a}^{\prime}, p\right)$ are entire in $\underline{a}^{\prime}$.

(c) For every $k \in \mathbb{Z}$, the matrix $M_{k}\left(\underline{a}^{\prime}, p\right)$ is holomorphic in $p \notin \mathbb{Z}$, and

$$
\widetilde{L}_{k}\left(\underline{a}^{\prime}, p\right)=L_{k}\left(\underline{a}^{\prime},-p\right)
$$

for all $p \notin \mathbb{Z}$ and all $\underline{a}^{\prime} \in \mathbb{C}^{m-1}$. Moreover, $\widetilde{L}_{k}$ extends analytically at $p \in \mathbb{N}^{\star}$.

(d) For every $k \in \mathbb{Z}$,

$$
M_{k}\left(\underline{a}^{\prime}, p\right)=M_{0}\left(\omega^{k} \cdot \underline{a}^{\prime}, p\right)\left(\begin{array}{cc}
\omega^{k s(p)} & 0 \\
\frac{2 i \pi k}{m} \lambda\left(\underline{a}^{\prime}, p\right) \omega^{k s(-p)} & \omega^{k s(-p)}
\end{array}\right) .
$$

In particular,

$$
M_{m}\left(\underline{a}^{\prime}, p\right)=M_{0}\left(\underline{a}^{\prime}, p\right) \mathfrak{M}\left(\underline{a}^{\prime}, p\right) .
$$

Proof. We only detail the proof for $p \notin \mathbb{Z}$.

(a) We deduce from (5-1) that

$$
W\left(\Phi_{k-1}, \Phi_{k}\right)=\operatorname{det}\left(M_{k}\right) W\left(f_{1}, f_{2}\right)
$$

where $W(.,$.$) denotes the Wronskian. From Lemma 2.5, we know that$

$$
W\left(\Phi_{k-1}, \Phi_{k}\right)=2(-1)^{k-1} \omega^{(k-1)(1-m / 2)+r\left(\omega^{k} \cdot \underline{a}\right)},
$$

while, by Theorem 4.2, taking the limit $x \rightarrow 0$ and using the fact that the Wronskian is $x$-independent, one easily gets

$$
W\left(f_{1}, f_{2}\right)=s(-p)-s(p)=-p .
$$

(b) From (5-1), we have

$$
\left(\begin{array}{c}
\Phi_{k-1} \\
\Phi_{k}
\end{array}\right)(x, \underline{a})=M_{k}\left(\underline{a}^{\prime}, p\right)\left(\begin{array}{c}
f_{1} \\
f_{2}
\end{array}\right)\left(x, \underline{a}^{\prime}, p\right)
$$


for all $k \in \mathbb{Z}$, with

$$
M_{k}\left(\underline{a}^{\prime}, p\right)=\left(\begin{array}{cc}
\beta_{k 1}\left(\underline{a}^{\prime}, p\right) & \beta_{k 2}\left(\underline{a}^{\prime}, p\right) \\
\beta_{k 3}\left(\underline{a}^{\prime}, p\right) & \beta_{k 4}\left(\underline{a}^{\prime}, p\right)
\end{array}\right)
$$

so that, in particular,

$$
\Phi_{k}(x, \underline{a})=\beta_{k 3}\left(\underline{a}^{\prime}, p\right) f_{1}\left(x, \underline{a}^{\prime}, p\right)+\beta_{k 4}\left(\underline{a}^{\prime}, p\right) f_{2}\left(x, \underline{a}^{\prime}, p\right) .
$$

Then

$$
\left(\begin{array}{c}
\Phi_{k-1} \\
\Phi_{k}
\end{array}\right)(\omega x, \omega \cdot \underline{a})=M_{k}\left(\omega \cdot \underline{a}^{\prime}, p\right)\left(\begin{array}{c}
f_{1} \\
f_{2}
\end{array}\right)\left(\omega x, \omega \cdot \underline{a}^{\prime}, p\right)
$$

By Proposition 5.2 and Corollary 4.7, we get

$$
\left(\begin{array}{c}
\Phi_{k} \\
\Phi_{k+1}
\end{array}\right)(x, \underline{a})=M_{k}\left(\omega \cdot \underline{a}^{\prime}, p\right)\left(\begin{array}{cc}
\omega^{s(p)} & 0 \\
0 & \omega^{s(-p)}
\end{array}\right)\left(\begin{array}{c}
f_{1} \\
f_{2}
\end{array}\right)\left(x, \underline{a}^{\prime}, p\right)
$$

so that

(5-8) $\Phi_{k}(x, \underline{a})=\omega^{s(p)} \beta_{k 1}\left(\omega \cdot \underline{a}^{\prime}, p\right) f_{1}\left(x, \underline{a}^{\prime}, p\right)+\omega^{s(-p)} \beta_{k 2}\left(\omega \cdot \underline{a}^{\prime}, p\right) f_{2}\left(x, \underline{a}^{\prime}, p\right)$.

Comparing (5-7) and (5-8), we obtain the announced form for $M_{k}$ with $\beta_{k 1}=L_{k}$ and $\beta_{k 2}=\widetilde{L}_{k}$, since $\left(f_{1}, f_{2}\right)$ is a fundamental system.

(c) We have

$$
M_{k}=-\frac{1}{p}\left(\begin{array}{cc}
\Phi_{k-1} & \Phi_{k-1}^{\prime} \\
\Phi_{k} & \Phi_{k}^{\prime}
\end{array}\right)\left(\begin{array}{cc}
f_{2}^{\prime} & -f_{1}^{\prime} \\
-f_{2} & f_{1}
\end{array}\right)
$$

so that the analytic properties of $M_{k}$ easily follow from the analytic properties of the $\Phi_{k}$ (see Theorem 2.7) and of $f_{1}$ and $f_{2}$ (see Theorem 4.2).

(d) The given statement follows from Proposition 5.2, by induction, inferring from (4-2) that $\mathfrak{N}\left(\omega \cdot \underline{a}^{\prime}, p\right)=\mathfrak{N}\left(\underline{a}^{\prime}, p\right)$.

In addition to Theorem 5.3, it is easy to show the following proposition (the special case where $\underline{a}^{\prime}=0$ follows from Remark 4.4).

Proposition 5.4. The restriction to $p \notin \mathbb{Z}$ of the function $L_{k}\left(\underline{a}^{\prime}, p\right)$ (respectively, $\left.\widetilde{L}_{k}\left(\underline{a}^{\prime}, p\right)\right)$ has a meromorphic continuation in $p$, with at most simple poles when $p \in \mathbb{N}$ (respectively, $-p \in \mathbb{N}$ ).

In the special case where $\underline{a}^{\prime}=0$, the restriction to $p \notin \mathbb{Z}$ of the function $L_{k}\left(\underline{a}^{\prime}, p\right)$ (respectively, $\widetilde{L}_{k}\left(\underline{a}^{\prime}, p\right)$ ) has a meromorphic continuation in $p$, with at most simple poles at $p \in m \mathbb{N}$ (respectively, $-p \in m \mathbb{N}$ ).

\section{Monodromy, Stokes-Sibuya and $0 \infty$ connection matrices}

We collect here the different results that we have on the monodromy, StokesSibuya, and $0 \infty$ connection matrices to obtain a set of functional relations. 
First functional equation. From the definition (5-1) of the $0 \infty$ connection matrices $M_{k}$, and the fundamental property (2-5) of the Stokes-Sibuya connection matrices, we have, for all $k \in \mathbb{Z}$,

$$
M_{k}\left(\begin{array}{c}
f_{1} \\
f_{2}
\end{array}\right)=\left(\begin{array}{c}
\Phi_{k-1} \\
\Phi_{k}
\end{array}\right)=\mathfrak{S}_{k}\left(\begin{array}{c}
\Phi_{k} \\
\Phi_{k+1}
\end{array}\right)=\mathfrak{S}_{k} M_{k+1}\left(\begin{array}{c}
f_{1} \\
f_{2}
\end{array}\right) .
$$

Since $\left(f_{1}, f_{2}\right)$ is a fundamental system, we have the following proposition.

Proposition 6.1. For all $k \in \mathbb{Z}$,

$$
\mathfrak{S}_{k}(\underline{a})=M_{k}\left(\underline{a}^{\prime}, p\right) M_{k+1}^{-1}\left(\underline{a}^{\prime}, p\right) .
$$

Using (6-1), we see that

$$
\mathfrak{S}_{0}(\underline{a}) \mathfrak{S}_{1}(\underline{a}) \ldots \mathfrak{S}_{m-1}(\underline{a})=M_{0}\left(\underline{a}^{\prime}, p\right) M_{m}^{-1}\left(\underline{a}^{\prime}, p\right) .
$$

Using (5-6), we obtain the following theorem.

Theorem 6.2. The Stokes-Sibuya connection matrices satisfy the functional relation

$$
\mathfrak{S}_{0}(\underline{a}) \mathfrak{S}_{1}(\underline{a}) \ldots \mathfrak{S}_{m-1}(\underline{a})=M_{0}\left(\underline{a}^{\prime}, p\right) \mathfrak{M}^{-1}\left(\underline{a}^{\prime}, p\right) M_{0}^{-1}\left(\underline{a}^{\prime}, p\right) .
$$

This functional relation is equivalent to formula (2-10) of Theorem 2.9. But this new formulation is interesting due to the following two corollaries.

Corollary 6.3. We have

$$
\operatorname{Tr}\left(\mathfrak{S}_{0}(\underline{a}) \mathfrak{S}_{1}(\underline{a}) \ldots \mathfrak{S}_{m-1}(\underline{a})\right)=-2 \cos (p \pi) .
$$

Proof. This follows from the fact that

$$
\operatorname{Tr}\left(M_{0}\left(\underline{a}^{\prime}, p\right) \mathfrak{M}^{-1}\left(\underline{a}^{\prime}, p\right) M_{0}^{-1}\left(\underline{a}^{\prime}, p\right)\right)=\operatorname{Tr}\left(\mathfrak{M}^{-1}\left(\underline{a}^{\prime}, p\right)\right)=-2 \cos (p \pi) .
$$

Corollary 6.4. We assume that $p \in \mathbb{N}^{\star}$. Then, with the notation of Theorem 4.2,

$$
\left.\mathfrak{S}_{0}(\underline{a}) \mathfrak{S}_{1}(\underline{a}) \ldots \mathfrak{S}_{m-1}(\underline{a})\right|_{\lambda\left(\underline{a}^{\prime}, p\right)=0}=(-1)^{p+1}\left(\begin{array}{ll}
1 & 0 \\
0 & 1
\end{array}\right) \text {. }
$$

Proof. From Corollary 4.7, we know that

$$
\mathfrak{M}\left(\underline{a}^{\prime}, p\right)=e^{2 i \pi s(p)}\left(\begin{array}{cc}
1 & 0 \\
2 i \pi \lambda\left(\underline{a}^{\prime}, p\right) & 1
\end{array}\right)
$$

with $2 s(p)=1+p$, so that

$$
\begin{aligned}
\left.M_{0}\left(\underline{a}^{\prime}, p\right) \mathfrak{M}^{-1}\left(\underline{a}^{\prime}, p\right) M_{0}^{-1}\left(\underline{a}^{\prime}, p\right)\right|_{\lambda\left(\underline{a}^{\prime}, p\right)=0} & =\left.\mathfrak{M}^{-1}\left(\underline{a}^{\prime}, p\right)\right|_{\lambda\left(\underline{a^{\prime}}, p\right)=0} \\
& =(-1)^{p+1}\left(\begin{array}{ll}
1 & 0 \\
0 & 1
\end{array}\right) .
\end{aligned}
$$




\section{Second functional equation.}

Theorem 6.5. We use the notation of Theorem 5.3.

(1) Suppose that $p \notin \mathbb{Z}$, and that a satisfies $\widetilde{L}_{0}\left(\omega^{k} \cdot \underline{a}^{\prime}, p\right) \neq 0$ for $k=0, \ldots, m-1$. (Note that $\widetilde{L}_{0}\left(\underline{a}^{\prime}, p\right)$ cannot be identically zero, so this is a generic hypothesis on $\underline{\text { a.) }}$ Then

$$
\frac{L_{0}\left(\underline{a}^{\prime}, p\right)}{\widetilde{L}_{0}\left(\underline{a}^{\prime}, p\right)}=-i \frac{\omega^{-3 / 2} \omega^{-(m+1) p / 2}}{p \sin (p \pi)} \sum_{k=0}^{m-1} \frac{\omega^{r\left(\omega^{k} \cdot a\right)+(k+1) p}}{\widetilde{L}_{0}\left(\omega^{k} \cdot \underline{a}^{\prime}, p\right) \widetilde{L}_{0}\left(\omega^{k+1} \cdot \underline{a}^{\prime}, p\right)} .
$$

(2) Suppose that $p \in \mathbb{N}^{\star}$, and that a satisfies $\widetilde{L}_{0}\left(\omega^{k} \cdot \underline{a}^{\prime}, p\right) \neq 0$ for $k=0, \ldots, m-1$. Then

$$
i p \pi \omega^{(3+p) / 2} \lambda\left(\underline{a}^{\prime}, p\right)=\sum_{k=0}^{m-1} \frac{\omega^{r\left(\omega^{k} \cdot \underline{a}, p\right)+(k+1) p}}{\widetilde{L}_{0}\left(\omega^{k} \cdot \underline{a}^{\prime}, p\right) \widetilde{L}_{0}\left(\omega^{k+1} \cdot \underline{a}^{\prime}, p\right)} .
$$

Proof. (1) Using (5-3) and (5-4) with $k=0$, we get

$$
\omega^{s(-p)} L_{0}\left(\underline{a}^{\prime}, p\right) \widetilde{L}_{0}\left(\omega \cdot \underline{a}^{\prime}, p\right)-\omega^{s(p)} L_{0}\left(\omega \cdot \underline{a}^{\prime}, p\right) \widetilde{L}_{0}\left(\underline{a}^{\prime}, p\right)=-\frac{2}{p} \omega^{-1+r(\underline{a})}
$$

and, more generally, for all $k=0, \ldots, m-1$,

$$
\omega^{s(-p)} L_{0}\left(\omega^{k} \cdot \underline{a}^{\prime}, p\right) \widetilde{L}_{0}\left(\omega^{k+1} \cdot \underline{a}^{\prime}, p\right)-\omega^{s(p)} L_{0}\left(\omega^{k+1} \cdot \underline{a}^{\prime}, p\right) \widetilde{L}_{0}\left(\omega^{k} \cdot \underline{a}^{\prime}, p\right)=-\frac{2}{p} \omega^{-1+r\left(\omega^{k} \cdot \underline{a}\right)} .
$$

We assume $\underline{a}$ generic so that $\widetilde{L}_{0}\left(\omega^{k} \cdot \underline{a}^{\prime}, p\right) \neq 0$ for all $k=0, \ldots, m-1$. The previous equalities then become

$$
\omega^{s(-p)} \frac{L_{0}\left(\omega^{k} \cdot \underline{a}^{\prime}, p\right)}{\widetilde{L}_{0}\left(\omega^{k} \cdot \underline{a}^{\prime}, p\right)}-\omega^{s(p)} \frac{L_{0}\left(\omega^{k+1} \cdot \underline{a}^{\prime}, p\right)}{\widetilde{L}_{0}\left(\omega^{k+1} \cdot \underline{a}^{\prime}, p\right)}=-\frac{2}{p} \frac{\omega^{-1+r\left(\omega^{k} \cdot \underline{a}\right)}}{\widetilde{L}_{0}\left(\omega^{k} \cdot \underline{a}^{\prime}, p\right) \widetilde{L}_{0}\left(\omega^{k+1} \cdot \underline{a}^{\prime}, p\right)} .
$$

From the holomorphy in $\underline{a}^{\prime}$ of $L_{0}$ and $\widetilde{L}_{0}$, and since $\omega^{m}=e^{2 i \pi}$, this can be written in the form

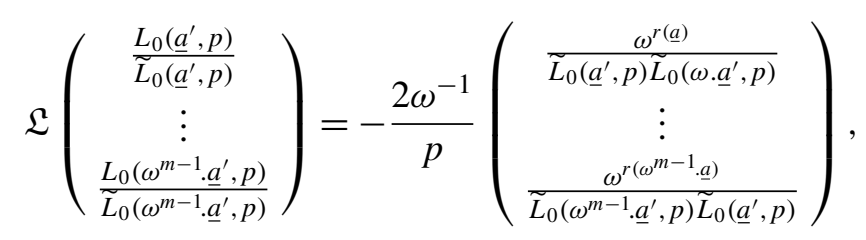

where

$$
\mathfrak{L}=\left(\begin{array}{ccccc}
\omega^{s(-p)} & -\omega^{s(p)} & 0 & \cdots & 0 \\
0 & \ddots & \ddots & \ddots & \vdots \\
\vdots & \ddots & \ddots & \ddots & 0 \\
0 & \cdots & 0 & \omega^{s(-p)} & -\omega^{s(p)} \\
-\omega^{s(p)} & 0 & \cdots & 0 & \omega^{s(-p)}
\end{array}\right)
$$


is an $m \times m$ circulant matrix whose determinant is $\omega^{m s(-p)}-\omega^{m s(p)}$. This determinant does not vanish because $s(p)-s(-p)=p$ is not an integer. The inverse of this matrix is also a circulant matrix, specifically

$$
\mathfrak{L}^{-1}=\frac{1}{\omega^{m s(-p)}-\omega^{m s(p)}}\left(\begin{array}{ccccc}
\omega^{(m-1) s(-p)} & \omega^{(m-2) s(-p)+s(p)} & \ldots & \omega^{s(-p)+(m-2) s(p)} & \omega^{(m-1) s(p)} \\
\omega^{(m-1) s(p)} & \omega^{(m-1) s(-p)} & \ldots & \ldots & \omega^{s(-p)+(m-2) s(p)} \\
\vdots & \vdots & \vdots & \vdots & \vdots \\
\omega^{(m-2) s(-p)+s(p)} & \ldots & \ldots & \omega^{(m-1) s(p)} & \omega^{(m-1) s(-p)}
\end{array}\right) .
$$

Since $s(p)-s(-p)=p$, this yields

$$
\frac{L_{0}\left(\underline{a}^{\prime}, p\right)}{\widetilde{L}_{0}\left(\underline{a}^{\prime}, p\right)}=-\frac{2 \omega^{-1} \omega^{(m-1) s(-p)}}{p\left(\omega^{m s(-p)}-\omega^{m s(p)}\right)} \sum_{l=0}^{m-1} \omega^{l p} \frac{\omega^{r\left(\omega^{l} \cdot \underline{a}\right)}}{\widetilde{L}_{0}\left(\omega^{l} \cdot \underline{a}^{\prime}, p\right) \widetilde{L}_{0}\left(\omega^{l+1} \cdot \underline{a}^{\prime}, p\right)}
$$

and also

$$
\frac{L_{0}\left(\underline{a}^{\prime}, p\right)}{\widetilde{L}_{0}\left(\underline{a}^{\prime}, p\right)}=i \frac{\omega^{-1} \omega^{(m-1) s(-p)}}{p \sin (p \pi)} \sum_{l=0}^{m-1} \omega^{l p} \frac{\omega^{r\left(\omega^{l} \cdot \underline{a}\right)}}{\widetilde{L}_{0}\left(\omega^{l} \cdot \underline{a}^{\prime}, p\right) \widetilde{L}_{0}\left(\omega^{l+1} \cdot \underline{a}^{\prime}, p\right)} .
$$

(2) We use (5-3) and (5-4) with $k=0$ and $p \in \mathbb{N}^{\star}$. Using also the fact that $\lambda\left(\omega \cdot \underline{a}^{\prime}, p\right)=\omega^{-p} \lambda\left(\underline{a}^{\prime}, p\right)$ (see Theorem 4.2), we get

$\mathfrak{L}\left(\begin{array}{c}\frac{L_{0}\left(\underline{a}^{\prime}, p\right)}{\underline{L}_{0}\left(\underline{a}^{\prime}, p\right)} \\ \vdots \\ \frac{L_{0}\left(\omega^{m-1} \cdot \underline{a}^{\prime}, p\right)}{\widetilde{L}_{0}\left(\omega^{m-1} \cdot \underline{a}^{\prime}, p\right)}\end{array}\right)=-\frac{2 \omega^{-1}}{p}\left(\begin{array}{c}\frac{\omega^{r(\underline{a})}}{\widetilde{L}_{0}\left(\underline{a}^{\prime}, p\right) \widetilde{L}_{0}\left(\omega \cdot \underline{a}^{\prime}, p\right)} \\ \vdots \\ \omega^{r\left(\omega^{m-1} \cdot \underline{a}\right)} \\ \widetilde{L}_{0}\left(\omega^{m-1} \cdot \underline{a}^{\prime}, p\right) \widetilde{L}_{0}\left(\underline{a}^{\prime}, p\right)\end{array}\right)+\frac{2 i \pi}{m} \omega^{s(-p)} \lambda\left(\underline{a}^{\prime}, p\right)\left(\begin{array}{c}1 \\ \vdots \\ \omega^{-(m-1) p}\end{array}\right)$

where $\mathfrak{L}$ is the previous circulant matrix. But now $\operatorname{det}(\mathfrak{L})=\omega^{m s(-p)}-\omega^{m s(p)}=0$, since $s(p)-s(-p)=p \in \mathbb{N}^{\star}$. It is straightforward to see that $\mathfrak{L}$ is of rank $m-1$, so that the compatibility condition is given by

$$
\operatorname{det}\left(\begin{array}{cccccc}
\omega^{s(-p)} & -\omega^{s(p)} & 0 & \cdots & 0 & \alpha_{0} \\
0 & \ddots & \ddots & \ddots & \vdots & \\
\vdots & \ddots & \ddots & \ddots & 0 & \vdots \\
0 & \cdots & 0 & \omega^{s(-p)} & -\omega^{s(p)} & \\
0 & 0 & \cdots & 0 & \omega^{s(-p)} & \alpha_{m-2} \\
-\omega^{s(p)} & 0 & \cdots & 0 & 0 & \alpha_{m-1}
\end{array}\right)=0
$$

where

$$
\alpha_{k}=-\frac{2 \omega^{-1}}{p} \frac{\omega^{r\left(\omega^{k} \cdot \underline{a}\right)}}{\widetilde{L}_{0}\left(\omega^{k} \cdot \underline{a}^{\prime}, p\right) \widetilde{L}_{0}\left(\omega^{k+1} \cdot \underline{a}^{\prime}, p\right)}+\frac{2 i \pi}{m} \omega^{s(-p)} \omega^{-k p} \lambda\left(\underline{a}^{\prime}, p\right) .
$$


This means that

$$
\begin{aligned}
& \omega^{(m-1) s(-p)} \alpha_{m-1}+\omega^{(m-1) s(p)} \alpha_{m-2} \\
& \quad+\omega^{(m-2) s(p)+s(-p)} \alpha_{m-3}+\cdots+\omega^{s(p)+(m-2) s(-p)} \alpha_{0}=0,
\end{aligned}
$$

and also, since $s(p)-s(-p)=p$,

$$
\alpha_{m-1}+\omega^{(m-1) p} \alpha_{m-2}+\omega^{(m-2) p} \alpha_{m-3}+\cdots+\omega^{p} \alpha_{0}=0 .
$$

Since $p$ is an integer, we eventually get

$$
i p \pi \omega^{\frac{3}{2}+\frac{p}{2}} \lambda\left(\underline{a}^{\prime}, p\right)=\frac{\omega^{r\left(\omega^{m-1} \cdot \underline{a}\right)}}{\widetilde{L}_{0}\left(\omega^{m-1} \cdot \underline{a}^{\prime}, p\right)}+\sum_{\widetilde{L}_{0}\left(\underline{a}^{\prime}, p\right)}^{m-2} \frac{\omega^{r\left(\omega^{k} \cdot \underline{a}^{\prime}, p\right)+(k+1) p}}{\widetilde{L}_{0}\left(\omega^{k} \cdot \underline{a}^{\prime}, p\right) \widetilde{L}_{0}\left(\omega^{k+1} \cdot \underline{a}^{\prime}, p\right)}
$$

which completes the proof.

Theorem 6.5 induces the following interesting result.

Corollary 6.6. The Stokes-Sibuya multiplier $C_{0}(\underline{a})$ satisfies the following conditions.

(1) If $m=1$,

$$
C_{0}(\underline{a})=-2 \cos (p \pi)
$$

for all $a \in \mathbb{C}$.

(2) If $m=2$,

$$
C_{0}(\underline{a}) \widetilde{L}_{0}\left(\omega \cdot \underline{a}^{\prime}, p\right)=-2 i e^{-i \pi a_{1} / 2} \cos \left(\frac{\pi}{2}\left(p+a_{1}\right)\right) \widetilde{L}_{0}\left(\underline{a}^{\prime}, p\right)
$$

for all $\underline{a}^{\prime} \in \mathbb{C}$ and $p \notin-\mathbb{N}$.

(3) If $m \geq 3$,

$$
\begin{aligned}
C_{0}(\underline{a}) \widetilde{L}_{0}\left(\omega \cdot \underline{a}^{\prime}, p\right)=\omega^{r(\underline{a})-1+(m / 4)}\left(\widetilde{L}_{0}\left(\underline{a}^{\prime}, p\right) \omega^{-r(\underline{a})+(1 / 2)-(m / 4)+(p / 2)}\right. \\
\left.+\widetilde{L}_{0}\left(\omega^{2} \cdot \underline{a}^{\prime}, p\right) \omega^{r(\underline{a})-(1 / 2)+(m / 4)-(p / 2)}\right)
\end{aligned}
$$

for all $\underline{a}^{\prime} \in \mathbb{C}^{m-1}$ and $p \notin-\mathbb{N}$.

Proof. Setting $k=0$ in formula (6-1) of Proposition 6.1 yields

$$
\mathfrak{S}_{0}(\underline{a})=M_{0}\left(\underline{a}^{\prime}, p\right) M_{1}^{-1}\left(\underline{a}^{\prime}, p\right) .
$$

Using (5-4) and (5-5), we obtain

$$
\begin{aligned}
& \left(\begin{array}{cc}
C_{0}(\underline{a}) & \widetilde{C}_{0}(\underline{a}) \\
1 & 0
\end{array}\right)= \\
& \left(\begin{array}{cc}
L_{0}\left(\underline{a}^{\prime}, p\right) & \widetilde{L}_{0}\left(\underline{a}^{\prime}, p\right) \\
\omega^{s(p)} L_{0}\left(\omega \cdot \underline{a^{\prime}}, p\right) & \omega^{s(-p)} \widetilde{L}_{0}\left(\omega \cdot \underline{\left.a^{\prime}, p\right)}\right.
\end{array}\right)\left(\begin{array}{cc}
\omega^{s(p)} L_{0}\left(\omega \cdot \underline{a}^{\prime}, p\right) & \omega^{s(-p)} \widetilde{L}_{0}\left(\omega \cdot \underline{a}^{\prime}, p\right) \\
\omega^{2 s(p)} L_{0}\left(\omega^{2} \cdot \underline{a^{\prime}}, p\right) & \omega^{2 s(-p)} \widetilde{L}_{0}\left(\omega^{2} \cdot \underline{a^{\prime}}, p\right)
\end{array}\right)^{-1}
\end{aligned}
$$


so that, with (5-3),

(6-5)

$$
C_{0}(\underline{a})=-\frac{p}{2} \omega^{1-r(\omega \cdot \underline{a})}\left(\omega^{-p} \frac{L_{0}\left(\underline{a}^{\prime}, p\right)}{\widetilde{L}_{0}\left(\underline{a}^{\prime}, p\right)}-\omega^{p} \frac{L_{0}\left(\omega^{2} \cdot \underline{a}^{\prime}, p\right)}{\widetilde{L}_{0}\left(\omega^{2} \cdot \underline{a}^{\prime}, p\right)}\right) \widetilde{L}_{0}\left(\underline{a}^{\prime}, p\right) \widetilde{L}_{0}\left(\omega^{2} \cdot \underline{a}^{\prime}, p\right) .
$$

We now apply formula (6-3) under the assumptions made in Theorem 6.5.

(1) When $m=1, \omega=e^{2 i \pi}$ and $r(\underline{a})=\frac{1}{4}$, formula (6-3) of Theorem 6.5 reduces to

$$
\frac{L_{0}(p)}{\widetilde{L}_{0}(p)}=-\frac{1}{p \sin (p \pi) \widetilde{L}_{0}(p) \widetilde{L}_{0}(p)} .
$$

This allows us to write (6-5) as

$$
C_{0}(\underline{a})=-\frac{\sin (2 p \pi)}{\sin (p \pi)}=-2 \cos (p \pi) .
$$

This result extends for all $\underline{a} \in \mathbb{C}$ by analytic continuation, since $C_{0}$ is entire in $\underline{a}$. (2) When $m=2$, we have $\omega=e^{i \pi}$ and $r(\underline{a})=-\frac{a_{1}}{2}$. Formula (6-3) of Theorem 6.5 becomes

$$
\begin{aligned}
\frac{L_{0}\left(\underline{a}^{\prime}, p\right)}{\widetilde{L}_{0}\left(\underline{a}^{\prime}, p\right)} & =\frac{\omega^{-3 p / 2}}{p \sin (p \pi)}\left(\frac{\omega^{p-a_{1} / 2}}{\widetilde{L}_{0}\left(\underline{a}^{\prime}, p\right) \widetilde{L}_{0}\left(\omega \cdot \underline{a}^{\prime}, p\right)}+\frac{\omega^{2 p+a_{1} / 2}}{\widetilde{L}_{0}\left(\omega \cdot \underline{a}^{\prime}, p\right) \widetilde{L}_{0}\left(\underline{a}^{\prime}, p\right)}\right), \\
& =2 \frac{\cos \left(\frac{\pi}{2}\left(p+a_{1}\right)\right)}{p \sin (p \pi)} \frac{1}{\widetilde{L}_{0}\left(\underline{a}^{\prime}, p\right) \widetilde{L}_{0}\left(\omega \cdot \underline{a}^{\prime}, p\right)} .
\end{aligned}
$$

This means that (6-5) becomes

$$
C_{0}(\underline{a})=-2 i e^{-i \pi \frac{a_{1}}{2}} \cos \left(\frac{\pi}{2}\left(p+a_{1}\right)\right) \frac{\widetilde{L}_{0}\left(\underline{a}^{\prime}, p\right)}{\widetilde{L}_{0}\left(\omega \cdot \underline{a}^{\prime}, p\right)} .
$$

The announced result follows by analytic continuation for all $\underline{a}^{\prime} \in \mathbb{C}$ and all $p \notin-\mathbb{N}$, since $C_{0}$ is entire in $\underline{a}$, while $\widetilde{L}_{0}$ is holomorphic in $\underline{a}^{\prime} \in \mathbb{C}$ and $p \notin-\mathbb{N}$.

(3) When $m>2$ we can write, by Theorem 6.5,

$$
\begin{array}{r}
C_{0}(\underline{a})=i \frac{\omega^{-\frac{1}{2}} \omega^{-(m-1) \frac{p}{2}-r(\omega \cdot \underline{a})}}{2 \sin (p \pi)}\left(\omega^{-p} \sum_{l=0}^{m-1} \frac{\omega^{l p+r\left(\omega^{l} \cdot \underline{a}\right)}}{\widetilde{L}_{0}\left(\omega^{l} \cdot \underline{a}^{\prime}, p\right) \widetilde{L}_{0}\left(\omega^{l+1} \cdot \underline{a}^{\prime}, p\right)}\right. \\
\left.-\omega^{p} \sum_{l=0}^{m-1} \frac{\omega^{l p+r\left(\omega^{l+2} \cdot \underline{a}\right)}}{\widetilde{L}_{0}\left(\omega^{l+2} \cdot \underline{a}\right) \widetilde{L}_{0}\left(\omega^{l+3} \cdot \underline{a^{\prime}}, p\right)}\right) \widetilde{L}_{0}\left(\underline{a}^{\prime}, p\right) \widetilde{L}_{0}\left(\omega^{2} \cdot \underline{a}^{\prime}, p\right)
\end{array}
$$


which equals

$$
\begin{aligned}
C_{0}(\underline{a})= & i \frac{\omega^{-\frac{1}{2}} \omega^{-(m-1) \frac{p}{2}-r(\omega \cdot \underline{a})}}{2 \sin (p \pi)}\left(\frac{\omega^{-p+r(\underline{a})}}{\widetilde{L}_{0}\left(\underline{a}^{\prime}, p\right) \widetilde{L}_{0}\left(\omega \cdot \underline{a}^{\prime}, p\right)}+\frac{\omega^{r(\omega \cdot a)}}{\widetilde{L}_{0}\left(\omega \cdot \underline{a}^{\prime}, p\right) \widetilde{L}_{0}\left(\omega^{2} \cdot \underline{a}^{\prime}, p\right)}\right. \\
& +\sum_{l=2}^{m-1} \frac{\omega^{(l-1) p+r\left(\omega^{l} \cdot \underline{a}\right)}}{\widetilde{L}_{0}\left(\omega^{l} \cdot \underline{a}^{\prime}, p\right) \widetilde{L}_{0}\left(\omega^{l+1} \cdot \underline{a}^{\prime}, p\right)}-\sum_{l=0}^{m-3} \frac{\omega^{(l+1) p+r\left(\omega^{l+2} \cdot \underline{a}\right)}}{\widetilde{L}_{0}\left(\omega^{l+2} \cdot \underline{a}^{\prime}, p\right) \widetilde{L}_{0}\left(\omega^{l+3} \cdot \underline{a}^{\prime}, p\right)} \\
& \left.-\frac{\omega^{(m-1) p+r(\underline{a})}}{\widetilde{L}_{0}\left(\underline{a}^{\prime}, p\right) \widetilde{L}_{0}\left(\omega \cdot \underline{a}^{\prime}, p\right)}-\frac{\omega^{m p+r(\omega \cdot \underline{a})}}{\widetilde{L}_{0}\left(\omega \cdot \underline{a}^{\prime}, p\right) \widetilde{L}_{0}\left(\omega^{2} \cdot \underline{a}^{\prime}, p\right)}\right) \widetilde{L}_{0}\left(\underline{a}^{\prime}, p\right) \widetilde{L}_{0}\left(\omega^{2} \cdot a^{\prime}, p\right) .
\end{aligned}
$$

The right-hand side of this equality simplifies to give

$$
\begin{aligned}
C_{0}(\underline{a})=\omega^{r(\underline{a})-1+(m / 4)}\left(\frac{\widetilde{L}_{0}\left(\underline{a}^{\prime}, p\right)}{\widetilde{L}_{0}\left(\omega \cdot \underline{a^{\prime}}, p\right)} \omega^{-r(\underline{a})+(1 / 2)-(m / 4)+(p / 2)}+\right. \\
\\
\left.\frac{\widetilde{L}_{0}\left(\omega^{2} \cdot \underline{a}^{\prime}, p\right)}{\widetilde{L}_{0}\left(\omega \cdot \underline{a}^{\prime}, p\right)} \omega^{r(\underline{a})-(1 / 2)+(m / 4)-(p / 2)}\right) .
\end{aligned}
$$

Again, the announced result follows by analytic continuation.

Third functional equation. In this subsection, we study a class of differential equations $\left(\mathfrak{E}_{m}\right)$ with higher symmetries. For that purpose, it will be useful to introduce new notations.

Notation 6.7. For $m, n \in \mathbb{N}^{\star}$, we define

$$
\underline{a}_{n}=\left(0, \ldots, 0, a_{n}, 0, \ldots, 0, a_{2 n}, 0, \ldots, 0, a_{j n}, 0 \ldots, 0, a_{n m}\right) \in \mathbb{C}^{n m},
$$

that is,

$$
\underline{a}_{n}=\left(a_{j}\right)_{1 \leq j \leq n m} \quad \text { so that } a_{j}=0 \text { if } j \neq 0 \quad(\bmod m) .
$$

For such an $\underline{a}_{n}$, we also define

$$
\underline{a}_{n}^{\prime}:=\left(a_{j}\right)_{1 \leq j \leq n m-1}
$$

and

$$
\begin{aligned}
& \underline{\tilde{a}}_{n}:=\left(\frac{a_{n}}{n^{2 / m}}, \frac{a_{2 n}}{n^{4 / m}}, \ldots, \frac{a_{n(m-1)}}{n^{2(m-1) / m}},-\frac{1}{4}+\frac{1+4 a_{n m}}{4 n^{2}}\right) \in \mathbb{C}^{m} \\
& \underline{\tilde{a}}_{n}^{\prime}:=\left(\frac{a_{n}}{n^{2 / m}}, \frac{a_{2 n}}{n^{4 / m}}, \ldots, \frac{a_{n(m-1)}}{n^{2(m-1) / m}}\right) \in \mathbb{C}^{m-1} .
\end{aligned}
$$

We shall consider in this subsection the differential equation

$\left(\mathfrak{E}_{n m}^{n}\right)$

$$
x^{2} \frac{d^{2}}{d x^{2}} \Phi\left(x, \underline{a}_{n}\right)=P_{n m}\left(x, \underline{a}_{n}\right) \Phi\left(x, \underline{a}_{n}\right) .
$$


This equation is a particular case of our main equation $\left(\mathfrak{E}_{n m}^{n}\right)$, but its higher symmetry will allow us to compare its Stokes-Sibuya and $0 \infty$ connection matrices with those of $\left(\mathfrak{E}_{m}\right)$, associated with the polynomial $P_{m}\left(x, \underline{\tilde{a}}_{n}\right)$ of lower order.

Lemma 6.8. If $\Phi$ satisfies the differential equation $\left(\mathfrak{E}_{n m}^{n}\right)$ with $n, m \in \mathbb{N}^{\star}$, then

$$
\Psi=\Psi\left(x, \underline{\tilde{a}}_{n}\right):=x^{(n-1) / 2 n} \Phi\left(\left(n^{2 / m} x\right)^{1 / n}, \underline{a}_{n}\right)
$$

satisfies the differential equation $\left(\mathfrak{E}_{m}\right)$ with $\underline{a}=\underline{\tilde{a}}_{n}$, that is,

$$
x^{2} \frac{d^{2}}{d x^{2}} \Psi\left(x, \underline{\tilde{a}}_{n}\right)=P_{m}\left(x, \underline{\tilde{a}}_{n}\right) \Psi\left(x, \underline{\tilde{a}}_{n}\right) .
$$

Proof. We consider the transformation

$$
\Psi\left(x, \underline{\tilde{a}}_{n}\right)=x^{\alpha} \Phi\left(\lambda x^{1 / n}, \underline{a}_{n}\right)
$$

with $\alpha=\frac{n-1}{2 n}$. Then

$$
x^{2} \Psi^{\prime \prime}\left(x, \underline{\tilde{a}}_{n}\right)=\frac{\lambda^{2}}{n^{2}} x^{\alpha+2 / n} \Phi^{\prime \prime}\left(\lambda x^{1 / n}, \underline{a}_{n}\right)+\alpha(\alpha-1) x^{\alpha} \Phi\left(\lambda x^{1 / n}, \underline{a}_{n}\right) .
$$

Assuming that $x^{2} \Phi^{\prime \prime}\left(x, \underline{a}_{n}\right)=P_{n m}\left(x, \underline{a}_{n}\right) \Phi\left(x, \underline{a}_{n}\right)$, one gets

$$
x^{2} \Psi^{\prime \prime}\left(x, \underline{\tilde{a}}_{n}\right)=\left(\frac{1}{n^{2}} P_{n m}\left(\lambda x^{1 / n}, \underline{a}_{n}\right)+\alpha(\alpha-1)\right) \Psi\left(x, \underline{\tilde{a}}_{n}\right) .
$$

Setting $\lambda=n^{2 / n m}$ completes the proof.

Notation 6.9. For $k \in \mathbb{Z}$, we denote by $C_{k}^{n}\left(\underline{a}_{n}\right)$ and $\widetilde{C}_{k}^{n}\left(\underline{a}_{n}\right)$ the Stokes-Sibuya coefficients associated with $\left(\mathfrak{E}_{n m}^{n}\right)$.

The above lemma induces the following corollary.

Corollary 6.10. The Stokes-Sibuya coefficients $C_{0}^{n}\left(\underline{a}_{n}\right)$ and $\widetilde{C}_{0}^{n}\left(\underline{a}_{n}\right)$ associated with equation $\left(\mathfrak{E}_{n m}^{n}\right)$ are related to the Stokes-Sibuya coefficients $C_{0}$ and $\widetilde{C}_{0}$ of equation $\left(\mathfrak{E}_{m}\right)$ by

$$
\begin{aligned}
& C_{0}^{n}\left(\underline{a}_{n}\right)=\omega^{(n-1) / 2 n} n^{(2 / m)-1-(4 / m) r_{m}\left(\underline{\tilde{a}}_{n}\right)} C_{0}\left(\underline{\tilde{a}}_{n}\right) \\
& \widetilde{C}_{0}^{n}\left(\underline{a}_{n}\right)=\omega^{(n-1) / n} \widetilde{C}_{0}\left(\underline{\tilde{a}}_{n}\right)
\end{aligned}
$$

where $\omega=e^{2 i \pi / m}$.

Proof. We denote by $\Phi_{0}$ the solution of $\left(\mathfrak{E}_{m}\right)$ that is characterized by its asymptotes

$$
T \Phi_{0}(x, \underline{a})=x^{r_{m}(\underline{a})} e^{-S_{m}(x, \underline{a})}(1+o(1))
$$

at infinity in the sector $\Sigma_{0}=\left\{|x|>0,|\arg (x)|<\frac{3 \pi}{m}\right\}$ (where $r_{m}=r$ and $S_{m}=S$ in Theorem 2.1). The Stokes-Sibuya coefficients $C_{0}$ and $\widetilde{C}_{0}$ are defined by

$$
\Phi_{0}\left(\omega^{-1} x, \omega^{-1} \cdot \underline{a}\right)=C_{0}(\underline{a}) \Phi_{0}(x, \underline{a})+\widetilde{C}_{0}(\underline{a}) \Phi_{0}(\omega x, \omega . \underline{a})
$$


with $\omega=e^{2 i \pi / m}$. We denote by $\Phi_{0}^{n}$ the analog for equation $\left(\mathfrak{E}_{n m}^{n}\right)$, so that

$$
T \Phi_{0}^{n}\left(x, \underline{a}_{n}\right)=x^{r_{n m}\left(\underline{a}_{n}\right)} e^{-S_{n m}\left(x, \underline{a}_{n}\right)}(1+o(1))
$$

at infinity in the sector $\Sigma_{0}^{n}=\left\{|x|>0,|\arg (x)|<\frac{3 \pi}{n m}\right\}$ (where $r_{n m}=r$ and $S_{n m}=S$ in Theorem 2.1), and

$$
\Phi_{0}^{n}\left(\omega_{n}^{-1} x, \omega_{n}^{-1} \cdot \underline{a}_{n}\right)=C_{0}^{n}\left(\underline{a}_{n}\right) \Phi_{0}^{n}\left(x, \underline{a}_{n}\right)+\widetilde{C}_{0}^{n}\left(\underline{a}_{n}\right) \Phi_{0}^{n}\left(\omega_{n} x ; \omega_{n} \cdot \underline{a}_{n}\right)
$$

with $\omega_{n}=e^{2 i \pi / m n}$. Introducing, by Lemma 6.8, the function

$$
\Psi_{0}\left(x, \underline{\tilde{a}}_{n}\right)=x^{(n-1) / 2 n} \Phi_{0}^{n}\left(\left(n^{2 / m} x\right)^{1 / n}, \underline{a}_{n}\right)
$$

we get a solution of $\left(\mathfrak{E}_{m}\right)$ such that

$$
T \Psi_{0}\left(x, \underline{\tilde{a}}_{n}\right)=n^{(2 / n m) r_{n m}\left(\underline{a}_{n}\right)} x^{(1 / n) r_{n m}\left(\underline{a}_{n}\right)+(n-1) / 2 n} e^{-S_{n m}\left(\left(n^{2 / m} x\right)^{1 / n}, \underline{a}_{n}\right)}(1+o(1))
$$

at infinity in the sector $\Sigma_{0}$. One easily checks that $S_{n m}\left(\left(n^{2 / m} x\right)^{1 / n}, \underline{a}_{n}\right)=S_{m}\left(x, \underline{\tilde{a}}_{n}\right)$ and $\frac{1}{n} r_{n m}\left(\underline{a}_{n}\right)=r_{m}\left(\underline{\tilde{a}}_{n}\right)-\frac{n-1}{2 n}$. This means that

$$
\Psi_{0}\left(x, \underline{\tilde{a}}_{n}\right)=n^{(2 / m) r_{m}\left(\underline{\tilde{a}}_{n}\right)-(n-1) / m n} \Phi_{0}\left(x, \underline{\tilde{a}}_{n}\right) .
$$

From (6-9), one observes that

$$
\begin{aligned}
\omega^{(n-1) / 2 n}\left(\omega^{-1} x\right)^{(n-1) / 2 n} \Phi_{0}^{n}\left(\left(n^{2 / m} \omega^{-1} x\right)^{1 / n}, \omega_{n}^{-1} \cdot \underline{a}_{n}\right) \\
=C_{0}^{n}\left(\underline{a}_{n}\right) x^{(n-1) / 2 n} \Phi_{0}^{n}\left(\left(n^{2 / m} x\right)^{1 / n}, \underline{a}_{n}\right) \\
\quad+\omega^{-(n-1) / 2 n} \widetilde{C}_{0}^{n}\left(\underline{a}_{n}\right)(\omega x)^{(n-1) / 2 n} \Phi_{0}^{n}\left(\left(n^{2 / m} \omega x\right)^{1 / n} ; \omega_{n} \cdot \underline{a}_{n}\right)
\end{aligned}
$$

so that, by (6-10),

$(6-13)$

$$
\omega^{\frac{n-1}{2 n}} \Psi_{0}\left(\omega^{-1} x, \omega^{-1} \cdot \underline{\tilde{a}}_{n}\right)=C_{0}^{n}\left(\underline{a}_{n}\right) \Psi_{0}\left(x, \underline{\tilde{a}}_{n}\right)+\omega^{-\frac{n-1}{2 n}} \widetilde{C}_{0}^{n}\left(\underline{a}_{n}\right) \Psi_{0}\left(\omega x, \omega \cdot \underline{\tilde{a}}_{n}\right) .
$$

Using (6-11), one can compare this last equation with (6-8) to complete the proof.

Lemma 6.8 can be used also to compare the $0 \infty$ connection matrices. We shall use the following notations.

Notation 6.11. For $k \in \mathbb{Z}$, we denote by $\widetilde{L}_{k}^{n}\left(\underline{a}_{n}^{\prime}, p\left(a_{m n}\right)\right)$ and $L_{k}^{n}\left(\underline{a}_{n}^{\prime}, p\left(a_{m n}\right)\right)$ the coefficients of the $0 \infty$ connection matrices associated to $\left(\mathfrak{E}_{n m}^{n}\right)$, with $p\left(a_{m n}\right)=$ $\left(1+4 a_{m n}\right)^{1 / 2}$.

Corollary 6.12. When $-p\left(a_{m n}\right) / n \notin \mathbb{N}$,

$$
\widetilde{L}_{0}^{n}\left(\underline{a}_{n}^{\prime}, p\left(a_{m n}\right)\right)=e^{i \pi(1-1 / n) / m} n^{-2 r\left(\underline{\tilde{a}}_{n}\right) / m+p\left(a_{m n}\right) / m n+1 / m-1} \widetilde{L}_{0}\left(\underline{\tilde{a}}_{n}^{\prime}, \frac{p\left(a_{m n}\right)}{n}\right) .
$$


Proof. We use the notation of Theorem 4.2. We introduce the solution $f_{1}(x, \underline{a})$ of $\left(\mathfrak{E}_{m}\right)$, where

$$
f_{1}\left(x, \underline{a}^{\prime}, p\left(a_{m}\right)\right)=x^{s\left(p\left(a_{m}\right)\right)} g_{1}\left(x, \underline{a}^{\prime}, p\left(a_{m}\right)\right),
$$

and we suppose that $p\left(a_{m}\right)=\left(1+4 a_{m}\right)^{1 / 2} \notin-\mathbb{N}$. Similarly, we denote by $f_{1}^{n}\left(x, \underline{a}_{n}^{\prime}, p\left(a_{m n}\right)\right)$ the solution of $\left(\mathfrak{E}_{n m}^{n}\right)$ which can be written as

$$
f_{1}^{n}\left(x, \underline{a}_{n}^{\prime}, p\left(a_{m n}\right)\right)=x^{s\left(p\left(a_{m n}\right)\right)} g^{n}\left(x, \underline{a}_{n}^{\prime}, p\left(a_{m n}\right)\right)
$$

if $p\left(a_{m n}\right) \notin-\mathbb{N}$. Following Lemma 6.8, we define

$$
F_{1}\left(x, \underline{\tilde{a}}_{n}\right)=x^{(n-1) / 2 n} f_{1}^{n}\left(\left(n^{2 / m} x\right)^{1 / n}, \underline{a}_{n}^{\prime}, p\left(a_{m n}\right)\right)
$$

which is a solution of $\left(\mathfrak{E}_{m}\right)$ with parameter $\underline{\tilde{a}}_{n}$. One easily checks that

$$
F_{1}\left(x, \underline{\tilde{a}}_{n}\right)=n^{2 s\left(p\left(a_{m n}\right)\right) / m n} f_{1}\left(x, \underline{\tilde{a}}_{n}^{\prime}, \frac{p\left(a_{m n}\right)}{n}\right) .
$$

In other words,

$$
f_{1}\left(x, \underline{\tilde{a}}_{n}^{\prime}, \frac{p\left(a_{m n}\right)}{n}\right)=n^{-2 s\left(p\left(a_{m n}\right)\right) / m n} x^{(n-1) / 2 n} f_{1}^{n}\left(\left(n^{2 / m} x\right)^{1 / n}, \underline{a}_{n}^{\prime}, p\left(a_{m n}\right)\right) .
$$

Note that this allows us to extend $f_{1}^{n}\left(x, \underline{a}_{n}^{\prime}, p\left(a_{m n}\right)\right)$ analytically for $\frac{p\left(a_{m n}\right)}{n} \notin-\mathbb{N}$, and this translates to the $\widetilde{L}_{k}^{n}\left(\underline{a}_{n}^{\prime}, p\left(a_{m n}\right)\right)$ as well.

We consider the $0 \infty$ connection matrices $M_{1}$ and $M_{1}^{n}$ associated with $\left(\mathfrak{E}_{m}\right)$ and $\left(\mathfrak{E}_{n m}^{n}\right)$ respectively. We have

$$
\widetilde{L}_{1}\left(\underline{a}^{\prime}, p\left(a_{m}\right)\right)=-\frac{1}{p\left(a_{m}\right)}\left(f_{1}\left(x, \underline{a^{\prime}}, p\left(a_{m}\right)\right) \Phi_{0}^{\prime}(x, \underline{a})-f_{1}^{\prime}\left(x, \underline{a^{\prime}}, p\left(a_{m}\right)\right) \Phi_{0}(x, \underline{a})\right)
$$

and

$(6-21)$

$$
\begin{aligned}
& \widetilde{L}_{1}^{n}\left(\underline{a}_{n}^{\prime}, p\left(a_{m n}\right)\right)= \\
& \quad-\frac{1}{p\left(a_{m n}\right)}\left(f_{1}^{n}\left(x, \underline{a}_{n}^{\prime}, p\left(a_{m n}\right)\right) \Phi_{0}^{n \prime}\left(x, \underline{a}_{n}\right)-f_{1}^{n \prime}\left(x, \underline{a}_{n}^{\prime}, p\left(a_{m n}\right)\right) \Phi_{0}^{n}\left(x, \underline{a}_{n}\right)\right)
\end{aligned}
$$

where $\Phi_{0}^{n}$ was defined in the proof of Corollary 6.10. By (6-10) and (6-11), we know that

$$
\Phi_{0}\left(x, \underline{\tilde{a}}_{n}\right)=n^{-2 r\left(\underline{\tilde{a}}_{n}\right) / m+(n-1) / m n} x^{(n-1) / 2 n} \Phi_{0}^{n}\left(\left(n^{2 / m} x\right)^{1 / n}, \underline{a}_{n}\right) .
$$


Equation (6-20), together with (6-19) and (6-22), yields

$$
\begin{aligned}
\widetilde{L}_{1}\left(\underline{\tilde{a}}_{n}^{\prime}, \frac{p\left(a_{m n}\right)}{n}\right)= & \\
\frac{1}{p\left(a_{m n}\right)} n^{\left(1-2 r\left(\underline{\tilde{a}}_{n}\right)-p\left(a_{m n}\right) / n\right) / m} & \left(f_{1}^{n \prime}\left(\left(n^{2 / m} x\right)^{1 / n}, \underline{a}_{n}^{\prime}, p\left(a_{m n}\right)\right) \Phi_{0}^{n}\left(\left(n^{2 / m} x\right)^{1 / n}, \underline{a}_{n}\right)\right. \\
& \left.-f_{1}^{n}\left(\left(n^{2 / m} x\right)^{1 / n}, \underline{a}_{n}^{\prime}, p\left(a_{m n}\right)\right) \Phi_{0}^{n \prime}\left(\left(n^{2 / m} x\right)^{1 / n}, \underline{a}_{n}\right)\right) .
\end{aligned}
$$

Comparing this with (6-21), we get

$$
\widetilde{L}_{1}^{n}\left(\underline{a}_{n}^{\prime}, p\left(a_{m n}\right)\right)=n^{\left(2 r\left(\underline{\tilde{a}}_{n}\right)+p\left(a_{m n}\right) / n-1\right) / m} \widetilde{L}_{1}\left(\underline{\tilde{a}}_{n}^{\prime}, \frac{p\left(a_{m n}\right)}{n}\right) .
$$

Using formula (5-5) of Theorem 5.3, we eventually find that

$$
\widetilde{L}_{0}^{n}\left(\underline{a}_{n}^{\prime}, p\left(a_{m n}\right)\right)=e^{i \pi(1-1 / n) / m} n^{\left(1-2 r\left(\underline{\tilde{a}}_{n}\right)+p\left(a_{m n}\right) / n\right) / m-1} \widetilde{L}_{0}\left(\underline{\tilde{a}}_{n}^{\prime}, \frac{p\left(a_{m n}\right)}{n}\right),
$$

since $r(\underline{a})+r(\omega \cdot \underline{a})=1-\frac{m}{2}$.

\section{Some applications}

Application for a special class. Some simplifications occur when $\underline{a}^{\prime}=0$, allowing to get the following proposition:

Proposition 7.1. We consider $\left(\mathfrak{E}_{m}\right)$ restricted to the case $\underline{a}^{\prime}=0$. Then

$$
\mathfrak{S}_{0}\left(0, a_{m}\right)=\left(\begin{array}{cc}
2 e^{-i \pi / m} \cos \left(\frac{p \pi}{m}\right) & -e^{-2 i \pi / m} \\
1 & 0
\end{array}\right)
$$

where $p=\left(1+4 a_{m}\right)^{1 / 2}$. Furthermore, for $\frac{p}{m} \notin \mathbb{Z}$, the $0 \infty$ connection matrix $M_{0}$ is given by

$$
M_{0}(0, p)=\left(\begin{array}{cc}
e^{\beta_{m}(-p) \frac{\omega^{-1 / 2}}{\sqrt{m \pi}} \Gamma\left(-\frac{p}{m}\right)} & e^{\beta_{m}(p) \frac{\omega^{-1 / 2}}{\sqrt{m \pi}} \Gamma\left(\frac{p}{m}\right)} \\
\omega^{s(p)} e^{\beta_{m}(-p) \frac{\omega^{-1 / 2}}{\sqrt{m \pi}} \Gamma\left(-\frac{p}{m}\right)} & \omega^{s(-p)} e^{\beta_{m}(p) \frac{\omega^{-1 / 2}}{\sqrt{m \pi}} \Gamma\left(\frac{p}{m}\right)}
\end{array}\right)
$$

where $s(p)=\frac{1+p}{2}$, while $\beta_{m}(p)$ is an odd function, entire in $p$, such that

$$
e^{\beta_{m}(k m)}= \pm m^{k}
$$

for all $k \in \mathbb{N}^{\star}$.

Remark 7.2. We shall see in Example 1 in the Appendix, by other means, that $\widetilde{L}_{0}(\underline{a})=-e^{i p \pi} \frac{\Gamma(p)}{\sqrt{\pi}}$ when $m=1$. Moreover, $r(\underline{a})=\frac{1}{4}$ for $m=1$. Applying Corollary 6.12 with $\underline{a}^{\prime}=0$, we deduce that

$$
\widetilde{L}_{0}(0, p)=e^{-i \pi / m} m^{p / m} e^{i \pi p / m} \frac{\Gamma\left(\frac{p}{m}\right)}{\sqrt{m \pi}}
$$


while

$$
L_{0}(0, p)=e^{-i \pi / m} m^{-p / m} e^{-i \pi p / m} \frac{\Gamma\left(-\frac{p}{m}\right)}{\sqrt{m \pi}} .
$$

Proof. We note that $\omega . \underline{a}=\underline{a}$ and $r(\underline{a})=\frac{1}{2}-\frac{m}{4}$ when $\underline{a}^{\prime}=0$. This has two consequences.

Firstly, when applying Corollary 6.6, one immediately gets

$$
C_{0}\left(0, a_{m}\right)=2 e^{-i \pi / m} \cos \left(\frac{p \pi}{m}\right)
$$

for $p \notin-\mathbb{N}$. Since $C_{0}(\underline{a})$ is an entire function in $\underline{a}$, the above formula extends to all $a_{m}$, by analytic continuation.

Secondly, formula (6-3) of Theorem 6.5 reduces into

$$
\frac{1}{L_{0}(0, p) \widetilde{L}_{0}(0, p)}=-\omega p \sin \left(\frac{p \pi}{m}\right),
$$

which resembles the Euler reflection formula $\frac{\pi}{\Gamma(z) \Gamma(-z)}=-z \sin (\pi z)$. Since, by Proposition 5.4, the restriction to $p \notin \mathbb{Z}$ of the function $L_{0}(0, p)$ (respectively, $\left.\widetilde{L}_{0}(0, p)\right)$ has a meromorphic continuation in $p$ with, at most, simple poles at $p \in$ $m \mathbb{N}$ (respectively, $-p \in m \mathbb{N}$ ), we can write

$$
L_{0}(0, p)=\alpha(p) \frac{\omega^{-1 / 2}}{\sqrt{m \pi}} \Gamma\left(-\frac{p}{m}\right)
$$

and

$$
\widetilde{L}_{0}(0, p)=\frac{1}{\alpha(p)} \frac{\omega^{-1 / 2}}{\sqrt{m \pi}} \Gamma\left(\frac{p}{m}\right)
$$

where $\alpha(p)$ is a nowhere vanishing entire function of $p$. In other words,

$$
\alpha(p)=e^{-\beta_{m}(p)}
$$

with $\beta_{m}(p)$ an entire function. Furthermore, also by Theorem 5.3, we know that $L_{0}\left(\underline{a}^{\prime},-p\right)=\widetilde{L}_{0}\left(\underline{a}^{\prime}, p\right)$. This means that $\beta_{m}$ can be chosen as an odd function.

Again by Theorem 5.3, we know that $\widetilde{L}_{0}\left(\underline{a}^{\prime}, p\right)$ extends analytically to $p \in \mathbb{N}^{\star}$. Moreover, when

$$
p=k m
$$

for some $k \in \mathbb{N}^{\star}$, then formula (6-4) of Theorem 6.5 gives

$$
\left.\widetilde{L}_{0}^{2}(0, p)\right|_{p=k m}=\left.(-1)^{k+1} \frac{m \omega^{-1}}{p \pi \lambda(0, p)}\right|_{p=k m .}
$$

By Remark 4.6, we know that

$$
\left.\lambda(0, p)\right|_{p=k m}=\frac{(-1)^{k+1}}{m^{2 k-1} k \Gamma(k)^{2}}
$$


and therefore

$$
\left.\widetilde{L}_{0}^{2}(0, p)\right|_{p=k m}=m^{2 k} \frac{\omega^{-1} \Gamma^{2}(k)}{m \pi},
$$

that is,

$$
\left.\widetilde{L}_{0}(0, p)\right|_{p=k m}= \pm m^{k} \frac{\omega^{-1 / 2} \Gamma(k)}{\sqrt{m \pi}} .
$$

Application when $m=2$, and consequences. We consider the case $m=2$, so that $\omega=e^{i \pi}$ and $r(\underline{a})=-\frac{a_{1}}{2}$.

On the one hand, Corollary 6.3 implies

$$
C_{0}(\underline{a}) C_{1}(\underline{a})+\widetilde{C}_{0}(\underline{a})+\widetilde{C}_{1}(\underline{a})=-2 \cos (p \pi)
$$

with $C_{1}(\underline{a})=C_{0}(\omega \cdot \underline{a})$, where, by formula (2-7) of Theorem 2.7,

$$
\widetilde{C}_{0}(\underline{a})=e^{-i \pi a_{1}} \quad \text { and } \widetilde{C}_{1}(a)=\widetilde{C}_{0}(\omega \cdot \underline{a})=e^{i \pi a_{1}} .
$$

This means that

$$
C_{0}(\underline{a}) C_{0}(\omega \cdot \underline{a})=-4 \cos \left(\left(p+a_{1}\right) \frac{\pi}{2}\right) \cos \left(\left(p-a_{1}\right) \frac{\pi}{2}\right) .
$$

On the other hand, we know by Corollary 6.6 that, for $\underline{a}$ generic,

$$
C_{0}(\underline{a})=-2 i e^{-i \pi a_{1} / 2} \cos \left(\left(p+a_{1}\right) \frac{\pi}{2}\right) \frac{\widetilde{L}_{0}\left(\underline{a}^{\prime}, p\right)}{\widetilde{L}_{0}\left(\omega \cdot \underline{a}^{\prime}, p\right)} .
$$

Also, by formula (6-4) of Theorem 6.5, we have, when $p \in \mathbb{N}^{\star}$ and for $\underline{a}^{\prime}=a_{1}$ generic,

$$
p \pi \lambda\left(\underline{a}^{\prime}, p\right)=\left.2 \frac{\cos \left(\left(p-a_{1}\right) \frac{\pi}{2}\right)}{\widetilde{L}_{0}\left(\underline{a}^{\prime}, p\right) \widetilde{L}_{0}\left(\omega \cdot \underline{a}^{\prime}, p\right)}\right|_{p \in \mathbb{N}^{\star}} .
$$

By Remark 4.6,

$$
\begin{aligned}
& \left.\lambda\left(\underline{a}^{\prime}, p\right)\right|_{p=0(\bmod 2)}=\frac{(-1)^{p+1}}{p \Gamma(p)^{2}} \prod_{k=1}^{p / 2}\left(a_{1}+2 k-1\right)\left(a_{1}-2 k+1\right) \\
& \left.\lambda\left(\underline{a}^{\prime}, p\right)\right|_{p=1(\bmod 2)}=\frac{(-1)^{p+1}}{p \Gamma(p)^{2}} a_{1} \prod_{k=1}^{(p-1) / 2}\left(a_{1}+2 k\right)\left(a_{1}-2 k\right),
\end{aligned}
$$

so that $\left.\widetilde{L}_{0}\left(\underline{a}^{\prime}, p\right) \widetilde{L}_{0}\left(\omega \cdot \underline{a}^{\prime}, p\right)\right|_{p \in \mathbb{N}^{\star}}$ equals

$$
\begin{cases}2(-1)^{(p+2) / 2} \frac{\cos \left(\frac{\pi}{2} a_{1}\right) \Gamma(p)^{2}}{\pi \prod_{k=1}^{p / 2}\left(a_{1}+2 k-1\right)\left(a_{1}-2 k+1\right)} & \text { for } p \text { even. } \\ 2(-1)^{(p+1) / 2} \frac{\sin \left(\frac{\pi}{2} a_{1}\right) \Gamma(p)^{2}}{\pi a_{1} \prod_{k=1}^{(p-1) / 2}\left(a_{1}+2 k\right)\left(a_{1}-2 k\right)} & \text { for } p \text { odd. }\end{cases}
$$


This can be also written as

$$
\left.\widetilde{L}_{0}\left(\underline{a}^{\prime}, p\right) \widetilde{L}_{0}\left(\omega \cdot \underline{a}^{\prime}, p\right)\right|_{p \in \mathbb{N}^{\star}}=-\frac{2^{-p+1} e^{i p \pi} \Gamma(p)^{2}}{\Gamma\left(\frac{p}{2}+\frac{a_{1}}{2}+\frac{1}{2}\right) \Gamma\left(\frac{p}{2}-\frac{a_{1}}{2}+\frac{1}{2}\right)} .
$$

At this point, we can use the following lemma, whose easy proof is left to the reader.

Lemma 7.3. When $p+a_{1}+1=-2 N$ with $N \in \mathbb{N}$, then, for $p \notin-\mathbb{N}^{\star}$,

$$
f_{1}\left(x, a_{1}, p\right)=x^{s(p)} e^{-x} \sum_{n=0}^{N} \frac{\Gamma(p+1) Q_{n}\left(a_{1}, p\right)}{\Gamma(n+p+1)} x^{n}
$$

where the $Q_{n}\left(a_{1}, p\right) \in \mathbb{C}\left[a_{1}, p\right]$ are defined by

$$
\begin{aligned}
& Q_{0}\left(a_{1}, p\right)=1 \\
& \left(a_{1}+p+1+2 n\right) Q_{n}\left(a_{1}, p\right)-(n+1) Q_{n+1}\left(a_{1}, p\right)=0 \quad \text { for } n \geq 0 .
\end{aligned}
$$

In particular, $f_{1}\left(x, a_{1}, p\right)=(-1)^{N} 2^{N} \frac{\Gamma(p+1)}{\Gamma(N+p+1)} \Phi_{0}(x, \underline{a})$.

This lemma implies that

$$
\widetilde{L}_{0}\left(\omega \cdot \underline{a}^{\prime}, p\right)=0 \quad \text { when } p+a_{1}+1 \in-2 \mathbb{N} \text { and } p \notin-\mathbb{N}^{\star},
$$

that is,

$$
\widetilde{L}_{0}\left(\underline{a}^{\prime}, p\right)=0 \quad \text { when } p-a_{1}+1 \in-2 \mathbb{N} \text { and } p \notin-\mathbb{N}^{\star} .
$$

Since the right-hand side of (7-2) has only simple zeros when $p+a_{1}+1 \in-2 \mathbb{N}$, we can write

$$
\left.\widetilde{L}_{0}\left(\underline{a}^{\prime}, p\right)\right|_{p \in \mathbb{N}^{\star}}=-\left.i 2^{-(p-1) / 2} e^{i \pi p / 2} \frac{\Gamma(p)}{\Gamma\left(\frac{p}{2}-\frac{a_{1}}{2}+\frac{1}{2}\right)} e^{\beta\left(a_{1}, p\right)}\right|_{p \in \mathbb{N}^{\star}}
$$

with $\beta\left(-a_{1}, p\right)=-\beta\left(a_{1}, p\right)$. Now when $p \notin \mathbb{Z}$, the coefficient $L_{0}\left(\underline{a}^{\prime}, p\right)$ can be derived from formula (6-3) of Theorem 6.5. This gives

$$
L_{0}\left(\underline{a}^{\prime}, p\right) \widetilde{L}_{0}\left(\omega \cdot \underline{a}^{\prime}, p\right)=2 \frac{\cos \left(\left(p+a_{1}\right) \frac{\pi}{2}\right)}{p \sin (p \pi)} .
$$

We recall also that $L_{0}\left(\underline{a}^{\prime}, p\right)$ can be derived from $\widetilde{L}_{0}\left(\underline{a}^{\prime}, p\right)$ just by changing $p$ into $-p$. Using (7-3) and the known analytic properties of $L_{0}\left(\underline{a}^{\prime}, p\right)$ and $\widetilde{L}_{0}\left(\underline{a}^{\prime}, p\right)$ described by Proposition 5.4, equation (7-5) shows that equation (7-4) can be extended to all $\left(\underline{a}^{\prime}, p\right) \in \mathbb{C}^{2}$ with $p \notin-\mathbb{N}^{\star}$, so that

$$
\widetilde{L}_{0}\left(\underline{a}^{\prime}, p\right)=-i 2^{-(p-1) / 2} e^{i \pi p / 2} \frac{\Gamma(p)}{\Gamma\left(\frac{p}{2}-\frac{a_{1}}{2}+\frac{1}{2}\right)} e^{\beta\left(a_{1}, p\right)}
$$


where $\beta$ can be chosen as an entire function satisfying

$$
\beta\left(-a_{1}, p\right)=-\beta\left(a_{1}, p\right) \quad \text { and } \quad \beta\left(a_{1},-p\right)=\beta\left(a_{1}, p\right) .
$$

Finally, (7-1) reduces to

$$
C_{0}(\underline{a})=-2 i e^{-i \pi a_{1} / 2} \cos \left(\left(p+a_{1}\right) \frac{\pi}{2}\right) \frac{\Gamma\left(\frac{p}{2}+\frac{a_{1}}{2}+\frac{1}{2}\right)}{\Gamma\left(\frac{p}{2}-\frac{a_{1}}{2}+\frac{1}{2}\right)} e^{2 \beta\left(a_{1}, p\right)} .
$$

To summarize:

Proposition 7.4. Suppose $m=2$. Then the Stokes-Sibuya multiplier $C_{0}$ may be written as

$$
\begin{aligned}
& C_{0}(\underline{a})=-2 i e^{-i \pi a_{1} / 2} \cos \left(\left(p+a_{1}\right) \frac{\pi}{2}\right) \frac{\Gamma\left(\frac{p}{2}+\frac{a_{1}}{2}+\frac{1}{2}\right)}{\Gamma\left(\frac{p}{2}-\frac{a_{1}}{2}+\frac{1}{2}\right)} e^{2 \beta\left(a_{1}, p\right)} \\
& \widetilde{C}_{0}(\underline{a})=e^{-i \pi a_{1}}
\end{aligned}
$$

where $\beta$ is an entire function satisfying $\beta\left(a_{1}, p\right)=\beta\left(a_{1},-p\right)=-\beta\left(-a_{1}, p\right)$. Moreover, for $p \notin \mathbb{Z}$, the coefficients of the $0 \infty$ connection matrix $M_{0}$ of Theorem 5.3 satisfy

$$
\begin{aligned}
& \widetilde{L}_{0}\left(a_{1}, p\right)=-i 2^{-(p-1) / 2} e^{i \pi p / 2} \frac{\Gamma(p)}{\Gamma\left(\frac{p}{2}-\frac{a_{1}}{2}+\frac{1}{2}\right)} e^{\beta\left(a_{1}, p\right)} \\
& L_{0}\left(a_{1}, p\right) \widetilde{L}_{0}\left(\omega a_{1}, p\right)=2 \frac{\cos \left(\left(p+a_{1}\right) \frac{\pi}{2}\right)}{p \sin (p \pi)}
\end{aligned}
$$

Remark 7.5. The above proposition is interesting since, for instance, it already provides the location of the zeroes of $C_{0}$ and of the other Stokes-Sibuya coefficients. However, one can be more precise using the Whittaker special functions. We shall see in Example 2 in the Appendix that

$$
\beta\left(a_{1}, p\right)=-\frac{1}{2} \ln (2) a_{1} .
$$

With this remark and Corollaries 6.10 and 6.12, Proposition 7.4 implies the following consequences.

Corollary 7.6. We consider the differential equation

$$
x^{2} \frac{d^{2}}{d x^{2}} \Phi=\left(x^{2 n}+a_{n} x^{n}+a_{2 n}\right) \Phi
$$

where $n \in \mathbb{N}^{\star}$. Then

$$
\begin{aligned}
& C_{0}^{n}\left(\underline{a}_{n}\right)=2 e^{-i \pi / 2 n} e^{-i \pi a_{n} / 2 n}\left(\frac{n}{2}\right)^{a_{n} / n} \frac{\Gamma\left(\frac{p}{2 n}+\frac{a_{n}}{2 n}+\frac{1}{2}\right)}{\Gamma\left(\frac{p}{2 n}-\frac{a_{n}}{2 n}+\frac{1}{2}\right)} \cos \left(\left(\frac{p}{2 n}+\frac{a_{n}}{2 n}\right) \pi\right) \\
& \widetilde{C}_{0}^{n}\left(\underline{a}_{n}\right)=-e^{-i \pi / n} e^{-i \pi a_{n} / n}
\end{aligned}
$$


where $p=\left(1+4 a_{2 n}\right)^{1 / 2}$. Moreover, when $p \notin-n \mathbb{N}$,

$$
\widetilde{L}_{0}^{n}\left(\underline{a}_{n}^{\prime}, p\right)=e^{-i \pi / 2 n} e^{i \pi p / 2 n}\left(\frac{n}{2}\right)^{a_{n} / 2 n+p / 2 n-1 / 2} \frac{\Gamma\left(\frac{p}{n}\right)}{\Gamma\left(\frac{p}{2 n}-\frac{a_{n}}{2 n}+\frac{1}{2}\right)} .
$$

Application when $m \geq 3$. As a matter of fact, we shall only discuss the cases $m=3$ and $m=4$ to show what kind of information we can extract from our analysis.

The case $m=3$. In a sense, this is the first interesting case, since no special function solution of $\left(\mathfrak{E}_{m}\right)$ is known for $m=3$. Here we have $\omega=e^{2 i \pi / 3}$ and $r(\underline{a})=-\frac{1}{4}$ is a constant function.

We first apply Corollary 6.3, to get a functional relation between the StokesSibuya multipliers:

$$
C_{0}(\underline{a}) C_{1}(\underline{a}) C_{2}(\underline{a})+\widetilde{C}_{0}(\underline{a}) C_{2}(\underline{a})+\widetilde{C}_{1}(\underline{a}) C_{0}(\underline{a})+\widetilde{C}_{2}(\underline{a}) C_{1}(\underline{a})=-2 \cos (p \pi)
$$

where, by (2-7) of Theorem 2.7,

$$
\widetilde{C}_{0}(\underline{a})=\widetilde{C}_{1}(\underline{a})=\widetilde{C}_{2}(\underline{a})=e^{i \pi / 3} .
$$

Applying Corollary 6.6, we find

$$
\widetilde{L}_{0}\left(\omega \cdot \underline{a}^{\prime}, p\right) C_{0}(\underline{a})=\omega^{-1 / 2}\left(\widetilde{L}_{0}\left(\underline{a}^{\prime}, p\right) \omega^{p / 2}+\widetilde{L}_{0}\left(\omega^{2} \cdot \underline{a}^{\prime}, p\right) \omega^{-p / 2}\right)
$$

for all $\underline{a}^{\prime} \in \mathbb{C}^{2}$ and $p \notin-\mathbb{N}$.

We concentrate on the case $p \in \mathbb{N}^{\star}$. By formula (6-4) of Theorem 6.5, we get

$$
\begin{aligned}
i p \pi \omega^{7 / 4+p / 2} \lambda\left(\underline{a}^{\prime}, p\right) \widetilde{L}_{0}\left(\underline{a}^{\prime}, p\right) \widetilde{L}_{0}\left(\omega \cdot \underline{a}^{\prime}, p\right) \widetilde{L}_{0}\left(\omega^{2} \cdot \underline{a}^{\prime}, p\right)= \\
\widetilde{L}_{0}\left(\omega \cdot \underline{a}^{\prime}, p\right)+\omega^{p} \widetilde{L}_{0}\left(\omega^{2} \cdot \underline{a}^{\prime}, p\right)+\omega^{2 p} \widetilde{L}_{0}\left(\underline{a}^{\prime}, p\right) .
\end{aligned}
$$

We now suppose, additionally, that $\underline{a}^{\prime}$ satisfies

$$
\lambda\left(\underline{a}^{\prime}, p\right) \widetilde{L}_{0}\left(\underline{a}^{\prime}, p\right) \widetilde{L}_{0}\left(\omega \cdot \underline{a}^{\prime}, p\right) \widetilde{L}_{0}\left(\omega^{2} \cdot \underline{a}^{\prime}, p\right)=0 .
$$

Using the fact that $\widetilde{L}_{0}\left(\omega \cdot \underline{a}^{\prime}, p\right)=0$ implies $\widetilde{L}_{0}\left(\underline{a}^{\prime}, p\right) \widetilde{L}_{0}\left(\omega^{2} \cdot \underline{a}^{\prime}, p\right) \neq 0$ necessarily (otherwise, one of the two $0 \infty$ connection matrices $M_{0}$ or $M_{1}$ is not invertible, which is absurd), equations (7-9) and (7-8) imply that

$$
C_{0}(\underline{a})=C_{1}(\underline{a})=C_{2}(\underline{a})=-\omega^{-1 / 2-3 p / 2}=(-1)^{p+1} e^{-i \pi / 3} .
$$

We summarize our results:

Proposition 7.7. Suppose $m=3$. Then the Stokes-Sibuya multiplier $C_{0}(\underline{a})$ satisfies the functional equation

$$
C_{0}(\underline{a}) C_{0}(\omega . \underline{a}) C_{0}\left(\omega^{2} \cdot \underline{a}\right)+e^{i \pi / 3}\left(C_{0}(\underline{a})+C_{0}(\omega . \underline{a})+C_{0}\left(\omega^{2} \cdot \underline{a}\right)\right)=-2 \cos (p \pi)
$$


with $p=\left(1+4 a_{3}\right)^{1 / 2}$ and $\omega=e^{2 i \pi / 3}$, whereas

$$
\widetilde{C}_{0}(\underline{a})=e^{i \pi / 3} .
$$

Moreover, when $a_{3}=\left(p^{2}-1\right) / 4$, with $p \in \mathbb{N}^{\star}$, then

$$
\lambda\left(\underline{a}^{\prime}, p\right) \widetilde{L}_{0}\left(\underline{a}^{\prime}, p\right) \widetilde{L}_{0}\left(\omega \cdot \underline{a}^{\prime}, p\right) \widetilde{L}_{0}\left(\omega^{2} \cdot \underline{a}^{\prime}, p\right)=0
$$

is equivalent to $C_{0}$ being a constant, specifically

$$
C_{0}=(-1)^{p+1} e^{-i \pi / 3} .
$$

We note that Proposition 7.7 can be derived from Corollary 6.4 if $\lambda\left(\underline{a}^{\prime}, p\right)=0$, while the particular case $a_{1}=a_{2}=0$ is given by Proposition 7.1.

For a given $p \in \mathbb{N}^{\star}$, the case $\lambda\left(\underline{a}^{\prime}, p\right)=0$ can be seen as an isomonodromic deformation condition, since both the monodromy at the origin and the Stokes structure are fixed. We get the following corollary.

Corollary 7.8. For $m=3$ and $p \in \mathbb{N}^{\star}$, the condition $\lambda\left(\underline{a}^{\prime}, p\right)=0$ is an isomonodromic deformation condition.

By computing $\lambda\left(\underline{a}^{\prime}, p\right)$ (see Proposition 4.5), one obtains, for example, from Proposition 7.7:

(1) If $p=1$, then $\lambda\left(\underline{a}^{\prime}, p\right)=a_{2}$. Therefore, for all $a_{1} \in \mathbb{C}$,

$$
C_{0}\left(a_{1}, 0,0\right)=e^{-i \pi / 3} \text {. }
$$

By a Tschirnhaus transformation, this case is equivalent to the Airy equation. This also means that $\widetilde{L}_{0}\left(a_{1}, 0,1\right)=\widetilde{L}_{0}(0,0,1)$ so that, by Remark 7.2,

$$
\begin{aligned}
& \widetilde{L}_{0}\left(a_{1}, 0,1\right)=e^{-i \pi / 3} 3^{1 / 3} e^{i \pi / 3} \frac{\Gamma\left(\frac{1}{3}\right)}{\sqrt{3 \pi}}, \\
& L_{0}\left(a_{1}, 0,1\right)=e^{-i \pi / 3} 3^{-1 / 3} e^{-i \pi / 3} \frac{\Gamma\left(-\frac{1}{3}\right)}{\sqrt{3 \pi}} .
\end{aligned}
$$

(2) If $p=2$, then $\lambda\left(\underline{a}^{\prime}, p\right)=-\frac{a_{2}^{2}}{2}+\frac{a_{1}}{2}$. We deduce that, for all $a_{2} \in \mathbb{C}$,

$$
C_{0}\left(a_{2}^{2}, a_{2}, \frac{3}{4}\right)=-e^{-i \pi / 3} \text {. }
$$

(3) If $p=3$, then $\lambda\left(\underline{a}^{\prime}, p\right)=\frac{a_{2}^{3}}{12}-\frac{a_{2} a_{1}}{3}+\frac{1}{3}$. Thus, for all $a_{2} \in \mathbb{C}^{\star}$,

$$
C_{0}\left(\frac{4+a_{2}^{3}}{4 a_{2}}, a_{2}, 2\right)=e^{-i \pi / 3} \text {. }
$$

Since $\lambda\left(\underline{a}^{\prime}, p\right)$ can be computed exactly for all fixed $p \in \mathbb{N}^{\star}$, it is natural to try to get more informations from equation (7-9). The result is a bit disappointing, as we now explain. 
We suppose that

$$
\widetilde{L}_{0}\left(\underline{a}^{\prime}, p\right) \widetilde{L}_{0}\left(\omega \cdot \underline{a}^{\prime}, p\right) \widetilde{L}_{0}\left(\omega^{2} \cdot \underline{a}^{\prime}, p\right) \neq 0 .
$$

We note that, by Remark 7.2,

$$
\widetilde{L}_{0}(0, p)=e^{-i \pi / 3} 3^{p / 3} e^{i \pi p / 3} \frac{\Gamma\left(\frac{p}{3}\right)}{\sqrt{3 \pi}},
$$

so that hypothesis (7-12) is valid for $\underline{a}^{\prime}$ in a neighbourhood of the origin. We can write (7-9) as

$$
y\left(\underline{a}^{\prime}, p\right)+\omega^{p} y\left(\omega \cdot \underline{a}^{\prime}, p\right)+\omega^{2 p} y\left(\omega^{2} \cdot \underline{a}^{\prime}, p\right)=-p \pi \omega^{1-p / 2} \lambda\left(\underline{a}^{\prime}, p\right)
$$

with

$$
y\left(\underline{a}^{\prime}, p\right)=\frac{1}{\widetilde{L}_{0}\left(\underline{a}^{\prime}, p\right) \widetilde{L}_{0}\left(\omega \cdot \underline{a}^{\prime}, p\right)} .
$$

Equation (7-13) can be thought of as a nonhomogeneous second order linear $q$ difference equation. Unfortunately, we are in the worst situation, when $q$ is a root of unity, so that solving (7-13) gives very little information. Indeed, we first observe that $-\frac{p \pi}{3} \omega^{1-p / 2} \lambda\left(\underline{a}^{\prime}, p\right)$ is a particular solution of (7-13), because $\lambda\left(\omega \cdot \underline{a}^{\prime}, p\right)=\omega^{-p} \lambda\left(\underline{a}^{\prime}, p\right)$. Therefore, by linearity of (7-13), one only needs to solve the homogeneous equation

$$
y\left(\underline{a}^{\prime}, p\right)+\omega^{p} y\left(\omega \cdot \underline{a}^{\prime}, p\right)+\omega^{2 p} y\left(\omega^{2} \cdot \underline{a}^{\prime}, p\right)=0
$$

in the space $\mathbb{C}\left\{a_{1}, a_{2}\right\}$. Writing $y\left(\underline{a}^{\prime}, p\right)=\sum_{k, l=0}^{\infty} b_{k, l} a_{1}^{k} a_{2}^{l}$, equation (7-14) is equivalent to

$$
\sum_{k, l=0}^{\infty}\left(1+\omega^{p+k+2 l}+\omega^{2 p+2 k+l}\right) b_{k, l} a_{1}^{k} a_{2}^{l}=0,
$$

since $\omega^{3}=e^{2 i \pi}$. Thus $y \in \mathbb{C}\left\{a_{1}, a_{2}\right\}$ is a solution of (7-14) provided that $b_{k, l}=0$ when $p+k+2 l=0(\bmod 3)$. This corresponds to a vector space of infinite dimension!

To end this subsection, we mention [Slavyanov 1996] for the numerical computations of the $0 \infty$ connection matrices.

The case $m=4$. When $m=4$, we have $r(\underline{a})=-\frac{1}{2}-\frac{1}{2} a_{2}+\frac{1}{8} a_{1}^{2}$ so that, by Theorem 2.7,

$$
\widetilde{C}_{0}(\underline{a})=i e^{-i \pi\left(a_{2}-a_{1}^{2} / 4\right) / 2} \text {. }
$$

Also, by Corollary 6.3,

$$
\begin{aligned}
C_{0}(\underline{a}) C_{1}(\underline{a}) C_{2}(\underline{a}) C_{3}(\underline{a}) & +\widetilde{C}_{0}(\underline{a}) C_{2}(\underline{a}) C_{3}(\underline{a})+\widetilde{C}_{1}(\underline{a}) C_{0}(\underline{a}) C_{3}(\underline{a}) \\
& +\widetilde{C}_{2}(\underline{a}) C_{0}(\underline{a}) C_{1}(\underline{a})+\widetilde{C}_{3}(\underline{a}) C_{1}(\underline{a}) C_{2}(\underline{a}) \\
& +\widetilde{C}_{0}(\underline{a}) \widetilde{C}_{2}(\underline{a})+\widetilde{C}_{1}(\underline{a}) \widetilde{C}_{3}(\underline{a})=-2 \cos (p \pi) .
\end{aligned}
$$


We already know, by Corollary 7.6 with $n=2$, that

$$
C_{0}\left(0, a_{2}, 0, a_{4}\right)=2 e^{-i \pi\left(a_{2}+1\right) / 4} \frac{\Gamma\left(\frac{p}{4}+\frac{a_{2}}{4}+\frac{1}{2}\right)}{\Gamma\left(\frac{p}{4}-\frac{a_{2}}{4}+\frac{1}{2}\right)} \cos \left(\left(p+a_{2}\right) \frac{\pi}{4}\right),
$$

with $p=\left(1+4 a_{4}\right)^{1 / 2}$.

Propositions similar to Proposition 7.7 can be obtained for every $m \geq 3$. In particular, for $m=4$, we show what happens for values of $\underline{a}$ such that $a_{4}=$ $\left(p^{2}-1\right) / 4$ with $p \in \mathbb{N}^{\star}$ and $\lambda\left(\underline{a}^{\prime}, p\right)=0$. Evaluating the last row of the product $\mathfrak{S}_{0}(\underline{a}) \mathfrak{S}_{1}(\underline{a}) \ldots \mathfrak{S}_{3}(\underline{a})$ and applying Corollary 6.4 , one gets

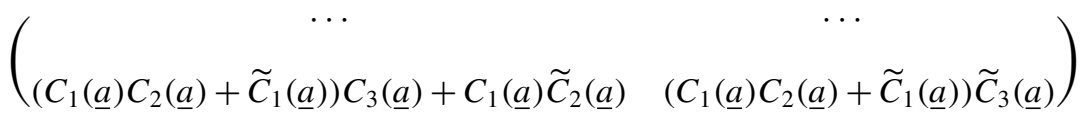

$$
\begin{aligned}
& =(-1)^{p+1}\left(\begin{array}{ll}
1 & 0 \\
0 & 1
\end{array}\right) \text {. }
\end{aligned}
$$

We have $\omega=e^{i \pi / 2}$ so that $\widetilde{C}_{2}(\underline{a})=\widetilde{C}_{0}\left(\omega^{2} \cdot \underline{a}\right)=\widetilde{C}_{0}(\underline{a})$. We thus get

$$
C_{0}(\underline{a}) C_{1}(\underline{a})+\widetilde{C}_{0}(\underline{a})=(-1)^{p+1} \widetilde{C}_{0}^{-1}(\underline{a})
$$

and

$$
\left(C_{0}(\underline{a}) C_{1}(\underline{a})+\widetilde{C}_{0}(\underline{a})\right) C_{2}(\underline{a})+C_{0}(\underline{a}) \widetilde{C}_{1}(\underline{a})=0 .
$$

Since $\widetilde{C}_{0}(\underline{a}) \widetilde{C}_{1}(\underline{a})=-1$, we obtain

$$
C_{0}(\underline{a})=(-1)^{p+1} C_{2}(\underline{a}), \quad C_{0}(\underline{a}) C_{1}(\underline{a})=(-1)^{p+1} \widetilde{C}_{0}^{-1}(\underline{a})-\widetilde{C}_{0}(\underline{a}) .
$$

Computing $\lambda\left(\underline{a}^{\prime}, p\right)$, one obtains for instance:

(1) If $p=1$, then $\lambda\left(\underline{a}^{\prime}, p\right)=a_{3}$. Therefore, for all $\left(a_{1}, a_{2}\right) \in \mathbb{C}^{2}$,

$$
\begin{aligned}
& C_{0}\left(a_{1}, a_{2}, 0,0\right)=C_{2}\left(a_{1}, a_{2}, 0,0\right), \\
& C_{0}\left(a_{1}, a_{2}, 0,0\right) C_{1}\left(a_{1}, a_{2}, 0,0\right)=-2 i \cos \left(\left(a_{2}-\frac{1}{4} a_{1}^{2}\right) \frac{\pi}{2}\right) .
\end{aligned}
$$

This case corresponds to the Weber equation. By a Tschirnhaus transformation, one can use equation (7-16) to get

$C_{0}\left(a_{1}, a_{2}, 0,0\right)=$

$$
2 e^{-i \pi / 4} e^{-i \pi\left(a_{2}-a_{1}^{2} / 4\right) / 4} \frac{\Gamma\left(\frac{a_{2}}{4}-\frac{1}{16} a_{1}^{2}+\frac{3}{4}\right)}{\Gamma\left(-\frac{a_{2}}{4}+\frac{1}{16} a_{1}^{2}+\frac{3}{4}\right)} \cos \left(\left(a_{2}-\frac{1}{4} a_{1}^{2}+1\right) \frac{\pi}{4}\right) .
$$

By the Euler reflection formula and the duplication formula for the Gamma function, one gets the usual well-known formula (see [Sibuya 1975]). 
Comparing this result with (7-16), it is tempting to conjecture that

$C_{0}\left(a_{1}, a_{2}, 0, a_{4}\right)=2 e^{-i \pi\left(a_{2}+1-a_{1}^{2} / 4\right) / 4} \frac{\Gamma\left(\frac{p}{4}+\frac{a_{2}}{4}-\frac{1}{16} a_{1}^{2}+\frac{1}{2}\right)}{\Gamma\left(\frac{p}{4}-\frac{a_{2}}{4}+\frac{1}{16} a_{1}^{2}+\frac{1}{2}\right)} \cos \left(\left(p+a_{2}-\frac{1}{4} a_{1}^{2}\right) \frac{\pi}{4}\right)$.

(This satisfies the functional relation (7-15), but also the known order-1 Taylor expansion; see [Bakken 1977]).

(2) If $p=2$, then $\lambda\left(\underline{a}^{\prime}, p\right)=-\frac{1}{2} a_{3}^{2}+\frac{1}{2} a_{2}$. We deduce that, for all $\left(a_{1}, a_{3}\right) \in \mathbb{C}^{2}$,

$$
\begin{aligned}
& C_{0}\left(a_{1}, a_{3}^{2}, a_{3}, \frac{3}{4}\right)=-C_{2}\left(a_{1}, a_{3}^{2}, a_{3}, \frac{3}{4}\right), \\
& C_{0}\left(a_{1}, a_{3}^{2}, a_{3}, \frac{3}{4}\right) C_{1}\left(a_{1}, a_{3}^{2}, a_{3}, \frac{3}{4}\right)=-2 \sin \left(\left(a_{3}^{2}-\frac{1}{4} a_{1}^{2}\right) \frac{\pi}{2}\right) .
\end{aligned}
$$

(3) If $p=3$, then $\lambda\left(\underline{a}^{\prime}, p\right)=\frac{1}{12} a_{3}^{3}-\frac{1}{3} a_{2} a_{3}+\frac{1}{3} a_{1}$. Thus, for all $\left(a_{2}, a_{3}\right) \in \mathbb{C}^{2}$,

$$
\begin{aligned}
& C_{0}\left(-\frac{1}{4} a_{3}^{3}+a_{2} a_{3}, a_{2}, a_{3}, 2\right)=C_{2}\left(-\frac{1}{4} a_{3}^{3}+a_{2} a_{3}, a_{2}, a_{3}, 2\right), \\
& \begin{array}{r}
C_{0}\left(-\frac{1}{4} a_{3}^{3}+a_{2} a_{3}, a_{2}, a_{3}, 2\right) C_{1}\left(-\frac{1}{4} a_{3}^{3}+a_{2} a_{3}, a_{2}, a_{3}, 2\right)= \\
-2 i \cos \left(\left(a_{2}-\frac{1}{4}\left(-\frac{1}{4} a_{3}^{3}+a_{2} a_{3}\right)^{2}\right) \frac{\pi}{2}\right) .
\end{array}
\end{aligned}
$$

\section{Appendix: Using special functions}

Example 1: a normal form of Heun's equation. We consider the equation

$$
x^{2} \Phi^{\prime \prime}=(x+a) \Phi
$$

This is the simplest case, when $m=1$. In this case, the Stokes-Sibuya connection matrix $\mathfrak{S}_{0}$ is given by Proposition 7.1. This proposition provides also the $0 \infty$ connection matrix $M_{0}$, up to an odd entire function of $p=(1+4 a)^{1 / 2}$, which we are going to compute here thanks to the fact that the above normal form $\left(\mathfrak{E}_{1}\right)$ of Heun's equation reduces to a modified Bessel equation. Indeed, setting

$$
t=2 x^{1 / 2}, \quad \Psi(t)=x^{-1 / 2} \Phi(x),
$$

equation $\left(\mathfrak{E}_{1}\right)$ is converted into the equation

$$
t^{2} \Psi^{\prime \prime}(t)+t \Psi^{\prime}(t)-\left(t^{2}+p^{2}\right) \Psi(t)=0, \quad p=(1+4 a)^{1 / 2},
$$

which is a modified Bessel equation of parameter $p$. Thus, we can use the wellknown special functions associated with the modified Bessel equation.

We assume that $p=(1+4 a)^{1 / 2} \notin \mathbb{Z}$. 
With the notations of Theorem 4.2, one easily gets the fundamental system of solutions $\left(f_{1}, f_{2}\right)$ of $\left(\mathfrak{E}_{1}\right)$ in the form $(\mathrm{A}-3)$

$$
\begin{aligned}
& f_{1}(x, p)=\Gamma(p+1) \sqrt{x} I_{p}(2 \sqrt{x}), \quad I_{p}(t)=\left(\frac{t}{2}\right)^{p} \sum_{n=0}^{+\infty} \frac{1}{n ! \Gamma(n+p+1)}\left(\frac{t}{2}\right)^{2 n}, \\
& f_{2}(x, p)=\Gamma(-p+1) \sqrt{x} I_{-p}(2 \sqrt{x}), \quad I_{-p}(t)=\left(\frac{t}{2}\right)^{-p} \sum_{n=0}^{+\infty} \frac{1}{n ! \Gamma(n-p+1)}\left(\frac{t}{2}\right)^{2 n},
\end{aligned}
$$

where $I_{p}$ is the modified Bessel function (or Bessel function of imaginary argument) of order $p$; see [Olver 1974].

Remark A.9. We recall that the functions $I_{p}$ and $I_{-p}$ are very closely connected to the Bessel functions $J_{p}$ and $J_{-p}$ of the first kind by

$$
I_{p}(t)=e^{-i p \pi / 2} J_{p}(i t), \quad I_{-p}(t)=e^{i p \pi / 2} J_{-p}(i t) ;
$$

see [Olver 1974].

Now, thanks to Theorem 2.1, there exists an unique solution $\Phi_{0}$ of $\left(\mathfrak{E}_{1}\right)$, asymptotic at infinity to $T \Phi_{0}(x, a)=e^{-2 \sqrt{x}} x^{1 / 4} \phi_{0}(x, a)$ with $\phi_{0} \in \mathbb{C}[a] \llbracket x^{-1 / 2} \rrbracket$ in the sector $-3 \pi<\arg (x)<3 \pi$. Specifically,

(A-4) $T \Phi_{0}(x, a)=e^{-2 \sqrt{x}} x^{1 / 4}\left(1+\sum_{n=1}^{+\infty} \frac{\left(4 p^{2}-1\right) \ldots\left(4 p^{2}-(2 n-1)^{2}\right)}{n ! 16^{n} x^{n / 2}}\right)$

in $\Sigma_{0}=\{|\arg (x)|<3 \pi\}$. Also, by Lemma 2.3 and Theorem 2.7, we have a fundamental system $\left(\Phi_{0}, \Phi_{1}\right)$ of solutions of $\left(\mathfrak{E}_{1}\right)$, where $\Phi_{1}$ is characterized by the asymptotic expansion at infinity

(A-5) $T \Phi_{1}(x, a)=e^{2 \sqrt{x}} \omega^{1 / 4} x^{1 / 4}\left(1+\sum_{n=1}^{+\infty}(-1)^{n} \frac{\left(4 p^{2}-1\right) \ldots\left(4 p^{2}-(2 n-1)^{2}\right)}{n ! 16^{n} x^{n / 2}}\right)$

in $\Sigma_{1}=\{|\arg (x)+2 \pi|<3 \pi\}$, where $\omega=e^{2 i \pi}$. As we shall see, these functions $\Phi_{0}$ and $\Phi_{1}$ can be expressed in terms of the MacDonald functions $K_{p}^{(1)}$ and $K_{p}^{(2)}$.

The MacDonald functions $K_{p}^{(1)}(t)$ and $K_{p}^{(2)}(t)$ are analytic functions in the variable $t$ for $t$ nonzero; they are linearly independent $\left(W\left(K_{p}^{(1)}, K_{p}^{(2)}\right)=\frac{\pi}{t}\right)$ and solutions of the modified Bessel equation (A-2). These functions are derived from the Hankel functions by the relationships

$$
\begin{aligned}
& K_{p}^{(1)}(t)=\frac{1}{2} i \pi e^{p i \pi / 2} H_{p}^{(1)}(i t), \\
& K_{p}^{(2)}(t)=\frac{1}{2} \pi e^{-p i \pi / 2} H_{p}^{(2)}(i t) .
\end{aligned}
$$


Furthermore, they admit, respectively, asymptotic expansions $T K_{p}^{(1)}$ and $T K_{p}^{(2)}$ of the form

(A-7) $\quad T K_{p}^{(1)}(t)=\left(\frac{\pi}{2 t}\right)^{1 / 2} e^{-t}\left(1+\sum_{n=1}^{+\infty} \frac{\left(4 p^{2}-1\right) \ldots\left(4 p^{2}-(2 n-1)^{2}\right)}{n ! 8^{n} t^{n}}\right)$ in $\widetilde{\Sigma}_{0}=\left\{|\arg (t)|<\frac{3 \pi}{2}\right\}$, and

(A-8) $T K_{p}^{(2)}(t)=\left(\frac{\pi}{2 t}\right)^{1 / 2} e^{t}\left(1+\sum_{n=1}^{+\infty}(-1)^{n} \frac{\left(4 p^{2}-1\right) \ldots\left(4 p^{2}-(2 n-1)^{2}\right)}{n ! 8^{n} t^{n}}\right)$

in $\widetilde{\Sigma}_{1}=\left\{|\arg (t)+\pi|<\frac{3 \pi}{2}\right\}$, when $t$ tends to infinity (see [Olver 1974]). Using $(\mathrm{A}-1)$ and $(\mathrm{A}-2)$, we deduce by uniqueness of $\Phi_{0}$ (respectively, $\Phi_{1}$ ), comparing (A-7) with (A-4) (respectively, (A-8) with (A-5)), that

$$
\begin{aligned}
& \Phi_{0}(x, a)=\frac{2}{\sqrt{\pi}} \sqrt{x} K_{p}^{(1)}(2 \sqrt{x}), \\
& \Phi_{1}(x, a)=\frac{2}{\sqrt{\pi}} \omega^{1 / 4} \sqrt{x} K_{p}^{(2)}(2 \sqrt{x}) .
\end{aligned}
$$

Recalling the connection formulas (see [Olver 1974]),

$$
\begin{aligned}
J_{p}(t) & =\frac{1}{2}\left(H_{p}^{(1)}(t)+H_{p}^{(2)}(t)\right), \\
J_{-p}(t) & =\frac{1}{2}\left(e^{i p \pi} H_{p}^{(1)}(t)+e^{-i p \pi} H_{p}^{(2)}(t)\right),
\end{aligned}
$$

we deduce, from Remark A.9 and (A-6),

$(\mathrm{A}-11)$

$$
I_{p}(t)=\frac{e^{-i p \pi}}{i \pi} K_{p}^{(1)}(t)+\frac{1}{\pi} K_{p}^{(2)}(t)
$$

$$
I_{-p}(t)=\frac{e^{i p \pi}}{i \pi} K_{p}^{(1)}(t)+\frac{1}{\pi} K_{p}^{(2)}(t) .
$$

Putting (A-3), (A-9), (A-10) and (A-11) together, we obtain

$$
\left(\begin{array}{l}
f_{1} \\
f_{2}
\end{array}\right)(x, p)=\left(\begin{array}{cc}
-i \frac{\Gamma(p+1)}{2 \sqrt{\pi}} e^{-i p \pi} & -i \frac{\Gamma(p+1)}{2 \sqrt{\pi}} \\
-i \frac{\Gamma(-p+1)}{2 \sqrt{\pi}} e^{i p \pi} & -i \frac{\Gamma(-p+1)}{2 \sqrt{\pi}}
\end{array}\right)\left(\begin{array}{l}
\Phi_{0} \\
\Phi_{1}
\end{array}\right)(x, a)
$$

where the matrix on the right-hand side of this equality is the inverse of the $0 \infty$ connection matrix $M_{1}$ (see (5-1)). By Proposition 5.2 we deduce

$$
M_{0}(p)=\left(\begin{array}{cc}
-e^{-i p \pi \frac{\Gamma(-p)}{\sqrt{\pi}}} & -e^{i p \pi \frac{\Gamma(p)}{\sqrt{\pi}}} \\
\frac{\Gamma(-p)}{\sqrt{\pi}} & \frac{\Gamma(p)}{\sqrt{\pi}}
\end{array}\right) .
$$

Remark A.10. This result is consistent with Proposition 7.1 and Remark 7.2. 
Example 2: a normal form of Whittaker's equation. We now focus on the equation

$$
x^{2} \Phi^{\prime \prime}=\left(x^{2}+a_{1} x+a_{2}\right) \Phi
$$

This equation reduces to the Whittaker equation. Indeed, the transformation

$$
x=\frac{t}{2}, \quad \phi(t)=\Phi(x)
$$

converts equation $\left(\mathfrak{E}_{2}\right)$ into

$$
\phi^{\prime \prime}(t)=\left(\frac{1}{4}+\frac{a_{1}}{2 t}+\frac{a_{2}}{t^{2}}\right) \phi(t)
$$

which is the Whittaker equation of parameters

$$
k=-\frac{a_{1}}{2} \quad \text { and } n=\frac{p}{2}=\left(\frac{1}{4}+a_{2}\right)^{1 / 2} .
$$

In what follows, we shall make a heavy use of the known properties of the special functions associated with the Whittaker equation, see for instance [Olver 1974].

Study near the origin. We assume here that $p=\left(1+4 a_{2}\right)^{1 / 2} \notin \mathbb{Z}$, that is, $2 n \notin \mathbb{Z}$. The fundamental system $\left(f_{1}, f_{2}\right)$ of solutions of Theorem 4.2 can be written

$$
\begin{aligned}
& f_{1}\left(x, a_{1}, p\right)=2^{-n-1 / 2} M_{k, n}(2 x), \\
& f_{2}\left(x, a_{1}, p\right)=2^{n-1 / 2} N_{k, n}(2 x)
\end{aligned}
$$

where

$$
\begin{aligned}
M_{k, n}(t) & =e^{-t / 2} t^{n+1 / 2} M\left(n-k+\frac{1}{2}, 2 n+1, t\right), \\
M(\alpha, c, t) & =\sum_{s=0}^{+\infty} \frac{(\alpha)_{s}}{(c)_{s}} \frac{t^{s}}{s !}, \\
N_{k, n}(t) & =e^{-t / 2} t^{n+1 / 2} N\left(n-k+\frac{1}{2}, 2 n+1, t\right), \\
N(\alpha, c, t) & =t^{1-c} M(1+\alpha-c, 2-c, t),
\end{aligned}
$$

with Pochhammer's notation: $(\alpha)_{0}=1,(\alpha)_{s}=\alpha(\alpha+1) \ldots(\alpha+s-1)$.

Remark A.11. The function $M_{k, n}$ is called a Whittaker function and $M(\alpha, c, t)$ is called the Kummer function (which is an entire function in $t$ ).

Study at infinity. In Theorem 2.1, the solution $\Phi_{0}$ of $\left(\mathfrak{E}_{2}\right)$ can be characterized by its asymptotics. Since $r(\underline{a})=-\frac{1}{2} a_{1}=k$ and $\omega=e^{i \pi}$, we have

$$
T \Phi_{0}(x, \underline{a})=x^{k} e^{-x} \phi_{0}(x, \underline{a}),
$$

with $\phi_{0}(x, \underline{a}) \in \mathbb{C}\left[a_{1}, a_{2}\right] \llbracket x^{-1} \rrbracket$ with constant term 1 in the sector $\left.\arg x \in\right]-\frac{3 \pi}{2}, \frac{3 \pi}{2}[$. 
In the same way, $\Phi_{1}$ is characterized by its asymptotics,

$$
T \Phi_{1}(x, \underline{a})=\omega^{-k} x^{-k} e^{x} \phi_{1}(x, \underline{a})
$$

where $\phi_{1}(x, \underline{a}) \in \mathbb{C}\left[a_{1}, a_{2}\right] \llbracket x^{-1} \rrbracket$ with constant term 1 in the same sector.

In fact, these two functions $\Phi_{0}$ and $\Phi_{1}$ can be expressed in terms of the functions $U$ and $V$ of the confluent hypergeometric equation

$$
t \frac{d^{2} f}{d t^{2}}+(c-t) \frac{d f}{d t}-\alpha f=0 .
$$

Proposition A.12.

$$
\Phi_{0}(x, \underline{a})=2^{-k} W_{k, n}(2 x),
$$

where

$$
\begin{aligned}
W_{k, n}(t) & =e^{-t / 2} t^{n+1 / 2} U\left(n-k+\frac{1}{2}, 2 n+1, t\right), \\
U(\alpha, c, t) & \sim t^{-\alpha} \sum_{s=0}^{+\infty} \frac{(-1)^{s}(1+\alpha-c)_{s}}{s ! t^{s}}
\end{aligned}
$$

in the sector $|\arg (t)|<\frac{3 \pi}{2}$, and

$$
\Phi_{1}(x, \underline{a})=i 2^{k} e^{n \pi i} V_{k, n}(2 x)
$$

where

$$
\begin{array}{r}
V_{k, n}(t)=e^{-\frac{t}{2}} t^{n+1 / 2} V\left(n-k+\frac{1}{2}, 2 n+1, t\right), \\
V(\alpha, c, t) \sim e^{t}\left(e^{i \pi} t\right)^{\alpha-c} \sum_{s=0}^{+\infty} \frac{(c-\alpha)_{s}(1-\alpha)_{s}}{s ! t^{s}}
\end{array}
$$

in the sector $|\arg (t)+\pi|<\frac{3 \pi}{2}$.

Remark A.13. $W_{k, n}$ is also called a Whittaker function; see [Olver 1974].

Connection formulas. We recall the connection formula

$$
M(\alpha, c, t)=\Gamma(c)\left(\frac{e^{-\alpha \pi i}}{\Gamma(c-\alpha)} U(\alpha, c, t)+\frac{e^{(c-\alpha) \pi i}}{\Gamma(\alpha)} V(\alpha, c, t)\right) ;
$$

see [Olver 1974]. Therefore

$$
M_{k, n}(2 x)=\frac{-i e^{(k-n) \pi i} \Gamma(2 n+1)}{\Gamma\left(n+k+\frac{1}{2}\right)} W_{k, n}(2 x)+\frac{i e^{(k+n) \pi i} \Gamma(2 n+1)}{\Gamma\left(n-k+\frac{1}{2}\right)} V_{k, n}(2 x),
$$

which means that 


$$
\text { (A-14) } \begin{aligned}
f_{1}\left(x, a_{1}, p\right)=-i e^{i \pi(k-n)} \frac{2^{k-n} \Gamma(2 n+1)}{\sqrt{2} \Gamma\left(n+k+\frac{1}{2}\right)} \Phi_{0}(x, \underline{a}) \\
+e^{i \pi k} \frac{2^{-k-n} \Gamma(2 n+1)}{\sqrt{2} \Gamma\left(n-k+\frac{1}{2}\right)} \Phi_{1}(x, \underline{a}) .
\end{aligned}
$$

Furthermore, thanks to the connection formula (see [Olver 1974])

$$
U(\alpha, c, t)=\frac{\Gamma(1-c)}{\Gamma(1+\alpha-c)} M(\alpha, c, t)-\frac{\Gamma(c) \Gamma(1-c)}{\Gamma(\alpha) \Gamma(2-c)} N(\alpha, c, t),
$$

we deduce that

$$
\begin{aligned}
N(\alpha, c, t) & =\frac{e^{(c-\alpha) \pi i} \Gamma(2-c)}{\Gamma(1+\alpha-c)} V(\alpha, c, t) \\
& +\left(\frac{\Gamma(\alpha) \Gamma(2-c) e^{-\alpha \pi i}}{\Gamma(1+\alpha-c) \Gamma(c-\alpha)}-\frac{\Gamma(\alpha) \Gamma(2-c)}{\Gamma(c) \Gamma(1-c)}\right) U(\alpha, c, t)
\end{aligned}
$$

so that

$$
\begin{aligned}
N_{k, n}(2 x) & =\left(\frac{e^{(n+k+1 / 2) \pi i} \Gamma(1-2 n)}{\Gamma\left(-n-k+\frac{1}{2}\right)}\right) V_{k, n}(2 x) \\
& +\left(\frac{\Gamma\left(n-k+\frac{1}{2}\right) \Gamma(1-2 n) e^{-(n-k+1 / 2) \pi i}}{\Gamma\left(-n-k+\frac{1}{2}\right) \Gamma\left(n+k+\frac{1}{2}\right)}-\frac{\Gamma\left(n-k+\frac{1}{2}\right) \Gamma(1-2 n)}{\Gamma(2 n+1) \Gamma(-2 n)}\right) W_{k, n}(2 x),
\end{aligned}
$$

that is,

$$
\begin{aligned}
& f_{2}\left(x, a_{1}, p\right)= \\
& \qquad \begin{array}{r}
\left(\frac{-i 2^{k+n} e^{(k-n) \pi i} \Gamma(1-2 n) \Gamma\left(n-k+\frac{1}{2}\right)}{\sqrt{2} \Gamma\left(n+k+\frac{1}{2}\right) \Gamma\left(-n-k+\frac{1}{2}\right)}-\frac{2^{k+n} \Gamma(1-2 n) \Gamma\left(n-k+\frac{1}{2}\right)}{\sqrt{2} \Gamma(2 n+1) \Gamma(-2 n)}\right) \Phi_{0}(x, \underline{a}) \\
+\left(\frac{2^{n-k} e^{k \pi i} \Gamma(1-2 n)}{\sqrt{2} \Gamma\left(-n-k+\frac{1}{2}\right)}\right) \Phi_{1}(x, \underline{a}) .
\end{array}
\end{aligned}
$$

Hence

$$
\text { (A-15) } \begin{aligned}
f_{2}\left(x, a_{1}, p\right)=-i e^{i \pi(n+k)} \frac{2^{k+n} \Gamma(1-2 n)}{\sqrt{2} \Gamma\left(-n+k+\frac{1}{2}\right)} \Phi_{0}(x, \underline{a}) \\
+e^{i \pi k} \frac{2^{n-k} \Gamma(1-2 n)}{\sqrt{2} \Gamma\left(-n-k+\frac{1}{2}\right)} \Phi_{1}(x, \underline{a}) .
\end{aligned}
$$

Formulas (A-14) and (A-15) yield the inverse of the $0 \infty$ connection matrix $M_{1}$. Going back to the variables $p$ and $a_{1}$, one gets 
(A-16) $M_{1}^{-1}\left(a_{1}, p\right)=\left(\begin{array}{cc}-i e^{i \pi\left(-a_{1}-p\right) / 2} \frac{2^{\left(-a_{1}-p\right) / 2} \Gamma(p+1)}{\sqrt{2} \Gamma\left(\frac{p}{2}-\frac{a_{1}}{2}+\frac{1}{2}\right)} & e^{-i \pi a_{1} / 2} \frac{2^{\left(a_{1}-p\right) / 2} \Gamma(p+1)}{\sqrt{2} \Gamma\left(\frac{p}{2}+\frac{a_{1}}{2}+\frac{1}{2}\right)} \\ -i e^{i \pi\left(-a_{1}+p\right) / 2} \frac{2^{\left(-a_{1}+p\right) / 2} \Gamma(1-p)}{\sqrt{2} \Gamma\left(-\frac{p}{2}-\frac{a_{1}}{2}+\frac{1}{2}\right)} & e^{-i \pi a_{1} / 2} \frac{2^{\left(a_{1}+p\right) / 2} \Gamma(1-p)}{\sqrt{2} \Gamma\left(-\frac{p}{2}+\frac{a_{1}}{2}+\frac{1}{2}\right)}\end{array}\right)$.

Using Proposition 5.2 we deduce that

(A-17) $\quad M_{0}\left(a_{1}, p\right)=\left(\begin{array}{cc}-i e^{-i \pi p / 2} \frac{2^{1+\left(-a_{1}+p\right) / 2} \Gamma(-p)}{\sqrt{2} \Gamma\left(-\frac{p}{2}-\frac{a_{1}}{2}+\frac{1}{2}\right)} & -i e^{i \pi p / 2} \frac{2^{1+\left(-a_{1}-p\right) / 2} \Gamma(p)}{\sqrt{2} \Gamma\left(\frac{p}{2}-\frac{a_{1}}{2}+\frac{1}{2}\right)} \\ \frac{2^{1+\left(a_{1}+p\right) / 2} \Gamma(-p)}{\sqrt{2} \Gamma\left(-\frac{p}{2}+\frac{a_{1}}{2}+\frac{1}{2}\right)} & \frac{2^{1+\left(a_{1}-p\right) / 2} \Gamma(p)}{\sqrt{2} \Gamma\left(\frac{p}{2}+\frac{a_{1}}{2}+\frac{1}{2}\right)}\end{array}\right)$.

Remark A.14. This result is consistent with Theorem 5.3. Also, with the notations of Theorem 5.3, we have found

$$
\widetilde{L}_{0}\left(a_{1}, p\right)=-i e^{i \pi p / 2} \frac{2^{1+\left(-a_{1}-p\right) / 2} \Gamma(p)}{\sqrt{2} \Gamma\left(\frac{p}{2}-\frac{a_{1}}{2}+\frac{1}{2}\right)} .
$$

In particular, when $a_{1}=0$ we get, using the Legendre duplication formula for the Gamma function,

$$
\widetilde{L}_{0}(0, p)=2^{p / 2} e^{i \pi p / 2} \frac{(-i)}{\sqrt{2 \pi}} \Gamma\left(\frac{p}{2}\right)
$$

a result which agrees also with Proposition 7.1 and Remark 7.2.

By formula (6-1) of Theorem 6.2, we have $\mathfrak{S}_{0}(\underline{a})=M_{0}\left(a_{1}, p\right) M_{1}^{-1}\left(a_{1}, p\right)$ and the result extends to $2 n \in \mathbb{Z}$ by analytic continuation, since $\mathfrak{S}_{0}$ is entire. We eventually get the following proposition.

Proposition A.15. We assume $m=2$. Then, for all $\underline{a}=\left(a_{1}, a_{2}\right) \in \mathbb{C}^{2}$, the StokesSibuya connection matrix $\mathfrak{S}_{0}$ is given by

$$
\mathfrak{S}_{0}(\underline{a})=\left(\begin{array}{cc}
-2 i e^{-i \pi a_{1} / 2} 2^{-a_{1}} \frac{\Gamma\left(\frac{p}{2}+\frac{a_{1}}{2}+\frac{1}{2}\right)}{\Gamma\left(\frac{p}{2}-\frac{a_{1}}{2}+\frac{1}{2}\right)} \cos \left(\left(\frac{p}{2}+\frac{a_{1}}{2}\right) \pi\right) & e^{-i \pi a_{1}} \\
1 & 0
\end{array}\right),
$$

where

$$
p=\left(1+4 a_{2}\right)^{1 / 2} \text {. }
$$

Moreover, when $p \notin \mathbb{Z}$, the $0 \infty$ connection matrix $M_{0}$ is given by formula (A-17).

\section{References}

[Bakken 1977] I. Bakken, "On the central connection problem for a class of ordinary differential equations, I and II”, Funkcial. Ekvac. 20:2 (1977), 115-156. MR 58:17365a Zbl 0386.34009

[Bender et al. 1999] C. M. Bender, S. Boettcher, and P. N. Meisinger, " $\mathscr{P} \mathscr{T}$-symmetric quantum mechanics”, J. Math. Phys. 40:5 (1999), 2201-2229. MR 2001d:81017 Zbl 1057.81512

[Bender et al. 2002] C. M. Bender, M. V. Berry, and A. Mandilara, "Generalized $P T$ symmetry and real spectra”, J. Phys. A 35:31 (2002), L467-L471. MR 2003m:81062 Zbl 02071787 
[Candelpergher et al. 1993a] B. Candelpergher, J.-C. Nosmas, and F. Pham, Approche de la résurgence, Hermann, Paris, 1993. MR 95e:34005 Zbl 0791.32001

[Candelpergher et al. 1993b] B. Candelpergher, J.-C. Nosmas, and F. Pham, "Premiers pas en calcul étranger", Ann. Inst. Fourier (Grenoble) 43:1 (1993), 201-224. MR 94f:34104 Zbl 0785.30017

[Costin 1998] O. Costin, "On Borel summation and Stokes phenomena for rank-1 nonlinear systems of ordinary differential equations", Duke Math. J. 93:2 (1998), 289-344. MR 99h:34003 Zbl 0948.34068

[Delabaere and Pham 1997] E. Delabaere and F. Pham, "Unfolding the quartic oscillator", Ann. Physics 261:2 (1997), 180-218. MR 99k:81065 Zbl 0977.34052

[Delabaere and Pham 1998] E. Delabaere and F. Pham, "Eigenvalues of complex Hamiltonians with PP T-symmetry, I”, Phys. Lett. A 250:1-3 (1998), 25-28. MR 2000k:81079

[Delabaere and Pham 1999] E. Delabaere and F. Pham, "Resurgent methods in semi-classical asymptotics”, Ann. Inst. H. Poincaré Phys. Théor. 71:1 (1999), 1-94. MR 2000f:34108 Zbl 0977.34053

[Delabaere and Trinh 2000] E. Delabaere and D. T. Trinh, "Spectral analysis of the complex cubic oscillator”, J. Phys. A 33:48 (2000), 8771-8796. MR 2001k:81064 Zbl 1044.81555

[Delabaere et al. 1993] É. Delabaere, H. Dillinger, and F. Pham, "Résurgence de Voros et périodes des courbes hyperelliptiques", Ann. Inst. Fourier (Grenoble) 43:1 (1993), 163-199. MR 94i:34115 Zbl 0766.34032

[Delabaere et al. 1997] E. Delabaere, H. Dillinger, and F. Pham, "Exact semiclassical expansions for one-dimensional quantum oscillators", J. Math. Phys. 38:12 (1997), 6126-6184. MR 99c:81041 Zbl 0896.34051

[Dieudonné 1968] J. Dieudonné, Calcul infinitésimal, Hermann, Paris, 1968. MR 37:2557 Zbl 0155.10001

[Dorey et al. 2001] P. Dorey, C. Dunning, and R. Tateo, "Spectral equivalences, Bethe ansatz equations, and reality properties in $\mathscr{P} \mathcal{T}$-symmetric quantum mechanics", J. Phys. A 34:28 (2001), 56795704. MR 2002i:81070 Zbl 0982.81021

[Écalle 1981] J. Écalle, "Les algèbres de fonctions résurgentes", Publ. Math. d'Orsay 81-05, Université de Paris-Sud, Orsay, 1981. MR 84h:30077a Zbl 0499.30034

[Écalle 1985] J. Écalle, "L'équation du pont et la classification analytique des objets locaux", Publ. Math. d'Orsay 85-05, Université de Paris-Sud, Orsay, 1985. MR 87k:32009 Zbl 0602.30029

[Fedoryuk 1983] M. V. Fedoryuk, Асимптотические методы для линеиных обыкновенных дифференциалных уравнении, Nauka, Moscow, 1983. MR 85m:34001 Zbl 0538.34001

[Gelfreich and Sauzin 2001] V. Gelfreich and D. Sauzin, "Borel summation and splitting of separatrices for the Hénon map", Ann. Inst. Fourier (Grenoble) 51:2 (2001), 513-567. MR 2002d:37045 Zbl 0988.37031

[Kawai and Takei 1994] T. Kawai and Y. Takei, "Secular equations through the exact WKB analysis”, pp. 85-102 in Analyse algébrique des perturbations singulières (Marseille and Luminy, 1991), vol. I, edited by L. Boutet de Monvel, Travaux en Cours 47, Hermann, Paris, 1994. MR 95i:81053 Zbl 0834.34068

[Loday-Richaud 1995] M. Loday-Richaud, "Solutions formelles des systèmes différentiels linéaires méromorphes et sommation”, Exposition. Math. 13:2-3 (1995), 116-162. MR 96i:34124 Zbl 0831. 34002

[Malgrange 1995] B. Malgrange, "Sommation des séries divergentes", Exposition. Math. 13:2-3 (1995), 163-222. MR 96i:34125 Zbl 0836.40004 
[Mullin 1968] F. E. Mullin, "On the regular perturbation of the subdominant solution to second order linear ordinary differential equations with polynomial coefficients", Funkcial. Ekvac. 11 (1968), 138. MR 39:3112 Zbl 0266.34052

[Olver 1974] F. W. J. Olver, Asymptotics and special functions, Academic Press, New York, 1974. MR 55:8655 Zbl 0303.41035

[Ramis 1993] J.-P. Ramis, Séries divergentes et théories asymptotiques, Panoramas et Synthèses (suppl. au Bull. Soc. Math. France 121), 1993. MR 95h:34074 Zbl 0830.34045

[Rasoamanana 2006] J.-M. Rasoamanana, Étude résurgente d'une classe d'équations différentielles de type Schrödinger, Ph.D. thesis, Université d'Angers, 2006.

[Reinhard 1982] H. Reinhard, Équations différentielles, Gauthier-Villars, Paris, 1982. Revised and updated translation: Differential equations: foundations and applications, North Oxford Academic, London, 1986. MR 84g:34002 Zbl 0495.34002

[Shin 2002] K. C. Shin, "On the reality of the eigenvalues for a class of $\mathscr{P} \mathcal{T}$-symmetric oscillators", Comm. Math. Phys. 229:3 (2002), 543-564. MR 2003g:34188 Zbl 1017.34083

[Sibuya 1975] Y. Sibuya, Global theory of a second order linear ordinary differential equation with a polynomial coefficient, North-Holland, Amsterdam, 1975. MR 58:6561 Zbl 0322.34006

[Slavyanov 1996] S. Y. Slavyanov, Asymptotic solutions of the one-dimensional Schrödinger equation, Translations of Mathematical Monographs 151. American Mathematical Society, Providence, RI, 1996. MR 97f:34001 Zbl 0847.34007

[Trinh 2005] D. T. Trinh, "Coefficients de Stokes du modèle cubique: point de vue de la résurgence quantique", Ann. Fac. Sci. Toulouse Math. (6) 14:1 (2005), 71-103.

[Voros 1983] A. Voros, "The return of the quartic oscillator: the complex WKB method", Ann. Inst. H. Poincaré Sect. A (N.S.) 39:3 (1983), 211-338. MR 86m:81051 Zbl 0526.34046

[Voros 2000] A. Voros, "Exercises in exact quantization", J. Phys. A 33:41 (2000), 7423-7450. MR 2002c:81053 Zbl 0991.81023

[Wasow 1965] W. Wasow, Asymptotic expansions for ordinary differential equations, Pure and Applied Mathematics 14, Interscience, New York, 1965. MR 34:3041 Zbl 0133.35301

Received March 5, 2004.

ERIC DELABAERE

DÉPARTEMENT DE MATHÉMATIQUES

UMR CNRS 6093

UNIVERSITÉ D' ANGERS

2 BOULEVARD LAVOISIER

49045 ANGERS CEDEX 01

FRANCE

eric.delabaere@univ-angers.fr

JEAN-MARC RASOAMANANA

DÉPARTEMENT DE MATHÉMATIQUES

UMR CNRS 6093

UNIVERSITÉ D' ANGERS

2 BOULEVARD LAVOISIER

49045 ANGERs CEDEX 01

FRANCE

jean-marc.rasoamanana@univ-angers.fr 